\title{
ESSAI D'UNE BIBLIOGRAPHIE DE LA REPRÉSENTATION ANALYTIQUE D'UNE FONCTION MONOGĖNE. ${ }^{1}$
}

PAR

EINAR HILLE

à Princeton, N. J., États Unis.

\section{Introduction.}

I. Le problème de la représentation analytique d'une fonction monogène se présente sous une forme variable avec la définition de la fonction donnée. D'une part il est possible de caractériser jusqu'à un certain point la fonction analytique par ses singularités. D'autre part on peut définir la fonction par une expression ánalytique, valable dans une certaine partie du plan complexe.

2. Dans le premier cas, nous sommes immédiatement en présence d'une question d'existence. Soit $D$ un domaine connexe arbitraire dans le plan complexe. $Y$ a-t-il une fonction monogène uniforme qui admet $D$ comme domaine d'existence? En fait, il y a une infinité de telles fonctions. Cela étant, nous nous demandons s'il est possible de se donner à l'avance l'allure de la fonction aux points accessibles de la frontière $F$ du domaine $D$. La réponse à cette question - qui ne forme guère un chapitre achevé - dépend de l'interprétation donnée au mot »allure». Dans le voisinage d'un point isolé de $F$ on peut se donner la partie principale de la série de Laurent. Les points non-isolés présentent des problèmes d'un caractère tout à fait différent et beaucoup plus difficile, des problèmes qui font appel aux méthodes les plus puissantes des théories modernes des ensembles et de l'intégration.

3. Pour construire effectivement une fonction monogène dont $D$ est le domaine d'existence, on peut se servir des séries de fonctions rationnelles. En

${ }^{1}$ La bibliographie suivante a été élaborée sur la demande de M. Mittag-Leffler.

La Rédaction.

1-2822. Acta mathematica. 52. Imprimé le 23 janvier 1929 , 
effet, toute fonction holomorphe et uniforme dans $D$ peut se représenter par une série de fonctions rationnelles, uniformément convergente dans tout domaine fermé intérieur à $D$. Lorsque le domaine est simplement connexe les fonctions rationnelles peuvent être remplacées par des polynomes.

4. Dans plusieurs questions le degré asymptotique d'approximation d'une fonction analytique par des polynômes de degré donné est d'une certaine importance. Ce degré est déterminé par la situation et la nature des points singuliers de la fonction donnée.

5. Le domaine de convergence d'une série de fonctions rationnelles donnée peut être formé de plusieurs domaines séparés qui n'ont aucun point intérieur commun. Dans chaque domaine la série représente une fonction monogène, mais les fonctions correspondant aux domaines différents sont distinctes dans le cas général. Il s'ensuit que des séries de ce caractère se prêtent à la construction de fonctions lacunaires. Mais elles ont également conduit à des considérations plus profondes qui ont donné naissance aux théories des fonctions monogènes nonanalytiques et des fonctions quasi-analytiques.

6. Nous venons d'observer qu'une expression analytique peut définir plusieurs fonctions analytiques. Or, il n'en est pas ainsi pour la série de Taylor, qui n'est pas sujette à cette ambiguité. Cette série est également remarquable par la simplicité de ses propriétés de convergence.

Le cercle de convergence de la série $\Sigma a_{n} z^{n}$ une fois déterminé, nous nous proposons de trouver l'allure de la fonction sur la périphérie et en dehors de son cercle de convergence.

7. Commençons par la question concernant la périphérie de ce cercle. Ici nous rencontrons une forme du problème d'Abel, que nous pouvons exprimer, sous une forme un peu généralisée et modifiée, comme il suit. Soit $u(t)=\Sigma u_{n}(t)$ une série convergente pour $\mathrm{o} \leqq t<\mathrm{I}$. Trouver des conditions suffisantes pour l'existence de $\lim _{t \rightarrow 1} u(t)$, et étudier les relations entre cette limite et la série formelle $\Sigma u_{n}(\mathrm{I})$.

La première question qui se pose est donc de trouver des conditions pour l'existence de valeurs limites sur le cercle de convergence. En un point $z=z_{0}$ situé sur ce cercle et où il existe une valeur limite - du moins pour un passage à la limite convenable - nous avons à trouver les relations entre cette limite et la série $\Sigma a_{n} z_{0}^{n}$. Or sur le cercle de convergence la série de Taylor est la somme formelle de deux séries trigonométriques conjuguées. C'est donc à la théorie des 
séries trigonométriques et en particulier à celle des séries de Fourier que nous devons nous adresser pour obtenir des renseignements sur cette question difficile.

8. Il y a une classe singulière de séries de Taylor, à savoir les séries nonprolongeables, pour lesquelles il n'y a aucun problème de représentation en dehors $\mathrm{du}$ cercle de convergence, du moins si l'on ne recourt pas au prolongement nonanalytique.

9. La méthode classique pour le prolongement analytique d'une série entière est d'une importance théorique fondamentale, mais elle ne se prête guère aux calculs numériques. Dans le cas où les coefficients $a_{n}$ sont les valeurs prises par une fonction analytique $g(z)$ - possédant des propriétés asymptotiques convenables - pour les valeurs entières de $z$, on peut souvent trouver un prolongement immédiat dans tout le plan. Il y a bien d'autres exemples de relations entre les coefficients et les singularités; nous rappelons les théorèmes de composition, aujourd'hui classiques, les recherches récentes sur l'influence du caractère arithmé: tique des coefficients, sur les lacunes, sur les suites hyper-convergentes et ainsi de suite.

ro. Dans le cas général où l'on ne sait rien sur les $a_{n}$, il faut utiliser d'autres méthodes. Nous savons déjà qu'une fonction monogène uniforme peut être représentée par une série de polynomes dans un domaine simplement connexe. Soit donc $f(z)=\Sigma a_{n} z^{n}$ une série prolongeable en dehors de son cercle de convergence. On peut trouver - et cela d'une infinité de manières différentes - des constantes $c_{m, n}$ telles que la série

$$
\sum_{n=0}^{\infty}\left\{\sum_{m=0}^{n} c_{m, n} a_{m} z^{m}\right\}
$$

converge vers $f(z)$ dans un domaine donné simplement connexe où cette fonction est monogène et uniforme. Si le domaine est lié d'une manière particulière aux points singuliers de $f(z)$, les $c_{m, n}$ peuvent être choisis indépendants des $a_{n}$. L'idée directrice dans ces recherches est la notion d'étoile, configuration géométrique dépendant d'une part des points singuliers de $f(z)$, d'autre part des propriétés analytiques d'une fonction génératrice. Sur la frontière de l'étoile de convergence le problème d'Abel se présente sous une forme assez difficile.

I I. Le problème de la détermination du prolongement analytique d'une série entière dans une étoile a des applications importantes, surtout dans la théorie des équations différentielles linéaires. 
I2. On peut regarder le problème de l'évaluation d'une série entière sur son cercle de convergence ou en dehors de ce cercle comme un cas spécial du problème de la sommation d'une série divergente. La théorie des séries sommables doit son existence surtout aux questions soulevées par le problème du prolongement analytique. Les recherches sur le cercle de convergence demandent des méthodes de sommation subtiles; en dehors du cercle il faut recourir à des méthodes plus puissantes mais aussi moins délicates. Il y a beaucoup de relations (d'équivalence, théorèmes tauberiens et ainsi de suite) entre les différentes méthodes de sommation qui sont très importantes pour les applications. Les fonctions sommatrices associées à ces méthodes diverses ont des propriétés analytiques intéressantes.

13. A côté de la théorie des séries sommables s'est développée une théorie des séries asymptotiques divergentes à laquelle se rattachent des recherches sur les fonctions de grands nombres, questions qui ont plusieurs applications dans la. théorie de la représentation analytique.

14. Tels sont les différents problèmes qui intervịennent dans la représentation analytique d'une fonction monogène et qui forment l'objet de la bibliographie suivante.

Il nous reste à dire quelques mots sur les limitations que nons nous sommes imposées dans notre travail. Nous n'avons pas essayé de retracer l'origine de notre problème jusqu'aux grands maîtres du calcul infinitésimal. Nous citons quelques travaux d'Abel, de Cauchy et de ses contemporains, mais, à ces travaux près, presque tous les ouvrages datent d'après 1876. Nous avons essayé de tenir compte de tous les travaux publiés jusqu'à la fin de l'année 1926, mais il est évident qu'une partie de la littérature des dernières années doit nous avoir échappé.

En général, nous nous sommes limités aux fonctions d'une variable complexe. Nous n'avons pas considéré la théorie des fonctions entières ou méromorphes, ni les recherches qui partent du théorème de $M$. Picard. Nous avons supprimé tous les travaux sur le développement d'une fonction en série de polynomes spéciaux (d'Hermite, de Laguerre, de Legendre etc.). Nous avons traité les séries spéciales d'interpolation (de Gauss, de Newton, de Stirling etc.) et les séries de facultés presque de la même manière. Enfin nous n'avons cité qu'une très petite partie des travaux sur les séries de Fourier et de Dirichlet; mais nous avons essayé de citer tous ceux des travaux modernes qui sont importants pour le problème d'Abel. 
Essai d'une bibliographie de la représentation analytique d'une fonction monogène.

15. Pour faciliter l'usage de notre bibliographie nous avons fait suivre chaque ouvrage d'une indication du contenu, sous la forme de quelques numéros de classification entre crochets. Pour la signification de ces nombres nous renvoyons le lecteur à la liste suivante.

\section{Liste des numéros de classification.}

\section{Représentation d'une fonction monogène uniforme.}

[ro]. Fonctions monogènes en général.

[I I]. Expressions analytiques représentant plusieurs fonctions monogènes.

[1 2]. Fonctions lacunaires. Lignes singulières.

[13]. Existence, représentation et propriétés des fonctions analytiques possédant des singularités données.

[14]. Séries de polynômes ou de fonctions rationnelles.

[I5]: Questions de degré d'approximation.

[16]: Fonctions monogènes non-analytiques.

\section{La série de Taylor.}

[20]. Séries entières en général.

[2 I]. Propriétés élémentaires.

[2 2]. L'allure sur le cerele de convergence. Problème d'Abel.

[23]. Séries non-prolongeables.

[24]. Relations entre coefficients et singularités.

[25]. Prolongement analytique dans une étoile.

[26]. L'allure du prolongement sur la frontière de l'étoile.

[27]. Applications diverses.

[28]. Prolongement non-analytique.

\section{Séries divergentes, sommables ou asymptotiques.}

[3o]. Questions générales sur la sommabilité.

[3I]. Méthodes spéciales de sommation.

[32]. Propriétés analytiques des fonctions sommatrices.

[33]. Théorèmes taubériens et questions analogues.

[34]. Séries semi-convergentes ou asymptotiques.

[35]. Fonctions de grands nombres. 


\section{Bibliographie.}

\section{N. H, Abel.}

(1) Untersuchungen über die Reihe

u. s. w.

$$
\mathbf{I}+\frac{m}{\mathrm{I}} x+\frac{m(m-\mathbf{I})}{\mathbf{I} \cdot \mathbf{2}} x^{2}+\frac{m(m-\mathbf{I})(m-2)}{\mathrm{I} \cdot 2 \cdot 3} x^{3}+\cdots
$$

Journal für Math. t. I (1826) p. 3 I I-339. Ostwald's Klassiker n:0 7 I, Leipzig, Engelman, I895, 46 p. $8^{\circ}$. Trad. française: Euvres complètes, t. I (nouv. éd. I 88 I) p. 2 I $9-250$. [22].

(2) Théorèmes et problèmes.

Journal für Math., t. 2 (I827) p. 286. (Euvres complètes, t. I (nouv. éd. I 88 I) p. 6 ×8-6I9. [24].

(3) Sommation de la série

$$
y=\varphi(0)+\varphi(\mathrm{I}) x+\varphi(2) x^{2}+\varphi(3) x^{3}+\cdots+\varphi(n) x^{n},
$$

$n$ étant un nombre entier positif fini ou infini, et $\varphi(n)$ une fonction algébrique rationnelle de $n$.

(Euvres completes, 1. 2 (nouv. éd. I 88I) p. I 4-18. [24].

(4) Sur les fonctions génératrices et leurs déterminantes.

(Euvres complètes, t. 2 (nouv. éd. I 88 I) p. $67-8$ I. [32].

\section{N. Abramesco (Abramescu).}

(1) Sur les développements en série suivant les inverses de polynomes donnés.

Comptes Rendus, t. I72 (r4 Mars I92 I) p. 649-65i. [I4].

(2) Sur les séries de polynomes à une variable complexe. Séries de M. Faber.

Buletinul soc. de ştiinţe din Cluj, t. I (I92r) p. 60-62. [I3, I4].

(3) Sur les séries de polynomes à une variable complexe. Journal de Math., série 9, t. I (1922) p. 77-84. [14].

(4) Sulle serie di polinomi di una variabile complessa.

Atti R. Accad. Naz. dei Lincei, Rendiconti. 5:e série, t. 3 I, i :er semestre (1922) p. $89-92 .[\mathrm{I} 4]$.

(5) Sulle serie di polinomi di Darboux e di Poincaré.

Atti R. Accad. Naz. dei Lincei, Rendiconti. 5:e série, t. 3 I, I:er semestre (I 922 ) p. $\mathbf{5}^{2}$ - I 53 . [ 14 ].

(6) Su una classe di serie di polinomi di una variabile complessa.

Atti R. Accad. Naz. dei Lincei, Rendiconti. 5:e série, t. 3 I, I :er semestre (I 922 ) p. $197-201$. [1 4].

(7) Sur les courbes de convergence des séries procédant suivant les inverses de polynomes donnés.

Comptes Rendus, t. I 80 (23 Février I 925 ) p. 566-569. [14]. 


\section{A. dell'Agnola.}

(I) Estensione di un teorema di Hadamard.

Atti del R. Ist. Veneto, t. 58 (1899) p. $5^{2} 5^{-539,669-677 . ~[24] . ~}$

(2) Sulle serie di polinomi.

Atti del R. Ist. Veneto, t. 60 (I goo-or) p. I 7 I-I 80. [1 $\left.4,2_{5}\right]$.

(3) Sulla serie di polinomi che rappresentano un ramo di funzione analitica monogena. Annali di Matematica. Serie III, tomo VI (I90I) p. $227-248$. [14, 25$]$.

(4) Analogie fra alcune serie di polinomi e le serie di potenze. Nota I e II. Atti R. Ist. Veneto, t. 64 (I904-05) p. 423-429, I I 43-I I 54. [I 4].

G. H. D'Ailly.

(r) Sur quelques propriétés des coefficients de certaines séries de Laurent. Thèse (Stockholm). Uppsala, Almquist \& Wiksell, i9r7, 53 p. $8^{\circ}$. [24].

\section{G. Alexitch.}

( I) Sur les valeurs d'une fonction analytique prises sur la circonférence du cercle à rayon unité.

Comptes Rendus, t. 183 (2 Août I926) p. 338-340. [22].

(2) Sur les valeurs limites d'une fonction analytique et sur les séries de puissances sur la circonférence $d u$ cercle de rayon un.

Bulletin sci. de l'École Polytechnique de Timișoara, t. I (1926) p. 198-2 I 2. [22].

\section{Amato.}

(r) Un criterio di convergenza e sua applicazione alla sommabilità secondo Riesz.

Rendiconto R. Accad. fis. e mat. di Napoli, 3:e série, t. 28 (1922) p. 39-50. $[3 x, 33]$.

\section{D. Ames.}

(i) Evaluation of slowly convergent series.

Annals of Math., série 2, t. 3(1902) p. I85-x92. [31].

\section{K. Ananda-Rau.}

(x) A note on a theorem of Mr. Hardy's. Proc. London Math. Soc. (2) I7 (1918) 334-336. [33].

(2) On Lambert's series.

Proceedings London Math. Soc. 2 :e série, t. I9 (I920) p. I-20. [I 2, 22, 3I, 33].

\section{A. F. Andersen.}

(I) Studier over Cesàro's Summabilitetsmetode. [ E Études sur la méthode de sommabilité de Cesàro.]

Thèse. Copenhague, I 921, I 00 p. $8^{\circ}$. $[22,31]$. 


\section{A. F. Andersen, H. Bohr et J. Mollerup.}

(I) Cesàro's Summabilitetsmetode med Anvendelse paa Fourier'ske og Dirichlet'ske Rækker. [ = La méthode de sommabilité de Cesàro avec application aux séries de Fourier et de Dirichlet.]

Copenhague, J. Gjellerup, r9 I9, $5 \mathrm{I}+20+25$ p. $8^{\circ}$. [3 I $]$.

\section{A. Angelesco.}

(I) Sur une classe de polynomes et une extension des séries de Taylor et de Laurent. Comptes Rendus, t. I76 (29 Janvier 1923) p. 275-278. [14].

\section{A. Anissimoff.}

(r) Sur la représentation et le prolongement des fonctions analytiques (en russe). Varsovie, 1892. [1 3, 25].

\section{P. Appell.}

(I) Sur certaines séries ordonnées par rapport aux puissances d'une variable. Comptes Rendus, t. 87 (28 Oct. 1 878 ) p. 689-692. [22].

(2) Sur les séries divergentes à termes positifs. Archiv d. Math. u. Physik, t. 64 (r879) p. $3^{87-392 . ~[22] . ~}$

(3) Développements en série d'une fonction holomorphe dans une aire limitée par des arcs de cercle.

Comptes Rendus, t. 94 (1. Mai I 882) p. I $\left.23^{-- \text {I } 240 . ~[13, ~ I ~} 4\right]$.

(4) Sur certains développements en série de puissances.

Bulletin Soc. Math. de France, t. I I (I $88_{2}-8_{3}$ ) p. $65-69$. [I3, I4].

(5) Développements en série dans une aire limitée par des arcs de cercle. Acta Math., t. 1. (1 $882-83)$ p. I $45-5_{5} 2$. [1 3,14$]$.

(6) Développements en série d'une fonction holomorphe dans une aire limitée par des arcs de cercle.

Math. Annalen, t. 2 I (i 883$)$ p. I I $8-$ I 24 . [ [ 3, I 4$]$.

(7) Sur les développements en série procédant suivant les inverses de polynomes donnés.

Comptes Rendus, t. I 57 (7 Juillet I913) p. 5-7. [1 4].

(8) Développement de $(x-y)^{-1}$ en série procédant suivant les inverses de polynomes donnés.

Comptes Rendus, t. I 57 (I Déc. I913) p. 1042-I043. [I4].

(9) Développements en série procédant suivant les inverses de polynomes donnés. Bulletin Sci. Math., Série 2, t. 37 (I9I3) p. 345-350. [14].

(ro) Sur les développements en série suivant les inverses de polynomes donnés. Bulletin Soc. Math. de France, t. 48 ( I920) p. I-8. [ [ 4 ].

(r r) Sur les développements en série suivant les inverses de polynomes. Bulletin Soc. Math. de France, t. 5I (1923) p. I89-19 I. [14]. 
Essai d'une bibliographie de la représentation analytique d'une fonction monogène.

\section{F. d'Arcais.}

(i) Sulle espressioni analitiche rappresentanti porzioni di funzioni analitiche diverse. Rivista di Mat., t. 5 (I895) p. I86-I89. [I I].

(2) Sulle funzioni di una variabile complessa.

Atti d. R. Ist. Veneto, 7:e série, t. 9 (1898) p. I048-1050. [I3].

G. d'Arone.

(1) Sur les fonctions à espaces lacunaires.

Bull. Soc. Math. de France, t. 23 (1895) p. 193- 194. [I 2].

\section{A. Arwin.}

(r) The Poisson integral and an analytic function on its circle of convergence. Annals of Math., 2:e série, t. 23 (I92 I-23) p. I4I-I43. [22].

\section{H. F. Baker.}

(1) An expression of $(\mathrm{I}-z)^{-1}$ by means of polynomials.

Proc. London Math. Soc. (2) 9 (1910) p. I22-125. [25].

\section{E. W. Barnes.}

(I) The theory of the gamma function. Messenger of Math., t. 29 (I900) p. 64-I 28 . [3 I, 34].

(2) A memoir on integral functions.

Phil. Transactions R. Soc. of London, série A, t. I99 (I902) p. 4II-500. Resumé dans les Proceedings R. Soc. of London, t. 69 (I902) p. I $21-I_{25}$. [I3, $25,31]$.

(3) The generalisation of the Maclaurin sum formula, and the range of its applicability.

Quarterly Journal of Math., t. 35. (I904) p. I75-188. [3I].

(4) The asymptotic expansion of the function $G(x, \theta)=\sum_{n=0}^{\infty} \frac{x^{n}}{\Gamma(n+\mathrm{I})(n+\theta)}$, and the singularities of $g(x, \theta)=\sum_{n=0}^{\infty} \frac{x^{n}}{n+\theta}$.

Quarterly Journal of Math., t. 37 (1906) p. 289-3 3 3. [24].

\section{Beke.}

(r) Egy tétel a hatványsorok maradéktagjára vonatkozólag. $[=\mathrm{Un}$ théorème sur le reste des séries entières.]

Math. és phys. lapok, t. I6 (I907) p. I41-144. [24].

2-2822. Acta mathematica. 52. Imprimé le 23 janvier 1929. 
(2) Vizsgálatok az analytikai függvények elmélete köréböl. [= Recherches sur la théorie des fonctions analytiques.]

Math. és termész. ért., t. 34 (I9I6) p. I-6I. [24].

(3) Adalékok a hatványsorok Hadamard- és Hurwitz-féle compositióihoz. [= Contributions aux compositions d'après Hadamard et Hurwitz des séries entières.]

Math. és termész. ért., t. 35 (I917) p. 87-1 I9. [24].

\section{G. Belardinelli.}

(r) La rappresentazione delle funzioni analitiche mediante la formula d'interpolazione di Newton.

Note e memorie di mat., Catania, t. 2 (I922) p. 53-72. [I3, I4].

(2) Sulla integrazione di una serie di funzioni razionali.

Atti R. Accad. Naz. dei Lincei, Rendiconti. 5:e série, t. 31, 2:e semestre (1 922 ) p. $178-180$. [I $4,16,28]$.

(3) Su una serie di funzioni razionali.

Atti R. Accad. Naz. dei Lincei. Rendiconti. 5:e série, t. 31, 2:e semestre (I922) p. $429-43$ I. [I 4 ].

(4) Sulle serie di funzioni.

Atti R. Accad. Naz. dei Lincei. Rendiconti. 5:e série, t. 32, r:er semestre (1923) p. $25 \mathrm{I}-255$. [1 2, I 4$]$.

(5) Sulle curve di convergenza delle serie di polinomi.

Rendiconti R. Ist. Lombardo, t. 58 (1925) p. 593-596. [I 4].

\section{J. Belinfante.}

(I) Over een generalisatie van Tauber's theorema betreffende machtreeksen. [= Sur une généralisation du théorème de $M$. Tauber concernant les séries entières.]

Verslagen K. Akad. v. Wetenschappen, Amsterdam, t. 32 (1923) p. $289-299$. $[22,33]$.

\section{F. Bernstein.}

(I) Die Übereinstimmung derjenigen beiden Summationsverfahren einer divergenten Reihe, welche von T. E. Stieltjes und E. Borel herrühren.

Jahresbericht d. deutsch. Math. Ver. t. 28 (I9I9) p. 50-63. [3I].

\section{S. Bernstein.}

( ) Sur la nature analytique des solutions des équations aux dérivées partielles du second ordre.

Math. Annalen, t. 59 (r9o4) p. $20-76$ (p. 36-5o). [I 4 ].

(2) Sur la valeur asymptotique de la meilleure approximation des fonctions analytiques.

Comptes Rendus, t. I55 (25 Nov. 1912) p. 1062-I065. [15]. 
Essai d'une bibliographie de la représentation analytique d'une fonction monogène. 11

(3) Sur la valeur asymptotique de la meilleure approximation des fonctions analytiques admettant des singularités données.

R. Ac. de Belgique. Bulletin Sci. I913 p. 76-90. [I5].

(4) Sur la meilleure approximation des fonctions analytiques possédant des singularités complexes.

Comptes Rendus, t. I 58 (6 Février I9 I 4) p. $467-469$. [I5].

(5) Sur la définition et les propriétés des fonctions analytiques d'une variable réelle. Math. Annalen, t. 75 (i 9 I4) p. 449-468. [I 5, 28].

(6) Leçons sur les propriétés extrémales et la meilleure approximation des fonctions analytiques d'une variable réelle.

Paris, Gauthier-Villars, 1926, $\mathrm{X}+207$ p. $8^{\circ}$. [I 5].

\section{Bernstein.}

(r) Sopra l'interpolazione a mezzo di funzioni olomorfe in un semipiano.

Atti R. Accad. Naz. dei Lincei. Rendiconti. 6:e série, t. 3 (r926) p. $73^{2}-73^{6}$. [24].

\section{A. Besikowitsch.}

(I) Über analytische Funktionen mit vorgeschriebenen Werten ihrer Ableitungen.

Math. Zeitschrift, t. 2 I (I92I) p. I I I- II8. [r3].

\section{Bieberbach.}

(I) Zwei Sätze über das Verhalten analytischer Funktionen in der Umgebung wesentlich singulärer Stellen.

Math. Zeitschrift, t. 2 ( 1918 ) p. I $58-$ r 70. [32].

(2) Über eine Vertiefung des Picardschen Satzes bei ganzen Funktionen endlicher Ordnung.

Math. Zeitschrift, t. 3 (r919) p. I75-rgo. [32].

(3) Auszug aus einem Briefe des Herrn Bieberbach an den Herausgeber. Acta Math., t. 42 (1920) p. 357-36r. [32].

(4) Neuere Untersuchungen über Funktionen von komplexen Variablen.

Encyklopädie der Math. Wissenschaften, volume II: 3, cahier 4, p. $379-53^{2}$, Leipzig. B. G. Teubner, I 92 I. [10, 20, 30].

\section{S. Bochner.}

(r) Über orthogonale Systeme analytischer Funktionen.

Math. Zeitschrift, t. I 4 (I922) p. I80-207. [I3, I 4$]$.

\section{K. Boehm.}

(I) Über die Entwickelung rationaler Funktionen nach Potenzen von Polynomen. Jahresbericht d. deutschen Math.-Vereinigung, t. 25 (I9 16) p. I 25- $3^{2}$. [13, 14$]$. 
(I) Sur la série de Dirichlet.

\section{H. Bohr.}

Comptes Rendus, t. I 48 (I I Janvier I909) p. 75-80. [3 I].

(2) Über die Summabilität Dirichletscher Reihen. Göttinger Nachrichten, 1909, p. 247--262. [3I].

(3) Bidrag til de Dirichlet'ske Rækkers Theori. [= Contribution à la théorie des séries de Dirichlet.]

Thèse. Copenhague, I9ro, IX +136 p. $4^{\circ}$. [3 I ]

(4) Om den Hadamard'ske "Hulsætning". [= Sur le théorème des lacunes de M. Hadamard.]

Matematisk Tidskrift B, I 9 I9, p. I 5-2 I. [23].

\section{É. Borel.}

( I) Sur une application d'un théorème de M. Hadamard.

Bull. Sci. Math., série 2, t. 18 (1894) p. 22-25. [24].

(2) Sur quelques points de la théorie des fonctions.

Comptes Rendus, t. I I 8 (I 2 Février r 894) p. 340-342. [I 2, I 4, I6].

(3) Sur quelques points de la théorie des fonctions.

Annales École Normale (3) t. I 2 (1895) p. 9-55.

Thèse, Paris, Gauthier-Villars (1894). [ 13, I 4, 16].

(4) Sur la sommation des séries divergentes.

Comptes Rendus, t. I 2 I (30 Déc. i 895) p. I I 25-I I 27 . [3 I].

(5) Sur la généralisation de la notion de limite et sur l'extension aux séries divergentes sommables du théorème d'Abel sur les séries entières.

Comptes Rendus, t. I 22 (I 3 Janvier I 896) p. 73-74. [2 2, 3I].

(6) Fondaments de la théorie des séries divergentes sommables. Journal de Math. (5) t. 2 (1896) p. 103-122. [22, 25, 31].

(7) Sur les séries de Taylor admettant leur cercle de convergence comme coupure. Journal de Math. (5) t. 2 (I 896) p. 44I-45I. [23, 24, 3I].

(8) Applications de la théorie des séries divergentes sommables. Comptes Rendus, t. I 22 (7 Avril I 896) p. 805-807. [31, 34].

(9) Sur la région de sommabilité d'un développement de Taylor. Comptes Rendus, t. I 23 (5 Oct. I 896 ) p. $548-549$. [25, 3 I].

(1 o) Sur les séries de Taylor.

Comptes Rendus, t. I 23 (I 4 Déc. I896) p. I 051 I-I052. [22, 23, 24$]$.

( I I) Sur les séries de Taylor. Lettre adressée à l'éditeur.

Acta Math., t. 2 I (1897) p. 243-247. [22, 24].

(12) Sur les développements des fonctions uniformes en série de Taylor. Comptes Rendus, t. I 27 (I4 Nov. i 898) p. 75 I. [24].

(13) Sur la recherche des singularités d'une fonction définie par un développement de Taylor.

Comptes Rendus, t. I 27 (I 2 Déc. i 898) p. Iоo I-I003. [24]. 
Essai d'une bibliographie de la représentation analytique d'une fonction monogène.

( 4 ) Sur les singularités des séries de Taylor.

Bull. Soc. Math. de France, t. 26 (1898) p. $238-248$. [24].

(15) Leçons sur la théorie des fonctions.

Paris, Gauthier-Villars I 898 ; IX + I $_{36}$ p., $8^{\circ}$. 2 2 :e édition I9I4, XI +259 p. $[12,13,14,16,28]$.

(16) Sur le prolongement des fonctions analytiques.

Comptes Rendus, t. r 28 (30 Janvier i 899) p. 283-284. [28].

( 7 ) Sur le calcul des séries de Taylor à rayon de convergence nul.

Comptes Rendus, t. I 28 (23 Mai i 899) p. I 28 I-I 283 . [25].

( 8$)$ Mémoire sur les séries divergentes.

Annales de l'École Normale. (3) t. I6. (r.899) p. 9-I3I. [22, 23, 24, 25, 27 , 3 I, 34].

(19) Addition au mémoire sur les séries divergentes.

Annales de l'École Normale (3) t. I6 (1899) p. I32-I36. [14, 28].

(20) Question 1477. L'intermédiaire des Mathématiciens t. VI. (1899) p. 74-75.Plusieurs réponses par Maillet (E.) dans t. I2 (I905) p. r4-I5; t. I4 (1907) p. $57-58$; t. I $5($ I 908$)$ p. 55. Remarques par la rédaction dans t. i 6 ( 909 ) p. $203-204$. [32].

(2 I) Sur les séries de fractions rationnelles. Comptes Rendus, t. I30 (I 7 Avril I900) p. I06 I-I064. [14].

(22) Sur la généralisation du prolongement analytique. Comptes Rendus, t. I30 (23 Avril i900) p. I I 5-i I I8. [28].

(23) Les séries absolument sommables, les séries $(M)$ et le prolongement analytique. Comptes Rendus, t. I3I (I9 Nov. I900) p. $830-832$. [25, 3I].

(24) Le prolongement analytique et les séries sommables. Math. Annalen, t. 55 (r9oo) p. $74-80 .[25,3 \mathrm{I}]$.

(25) Sur le prolongement analytique de la série de Taylor. Bull. Soc. Math. de France, t. 28 (1900) p. 200. [25].

(26) Sur les séries de polynomes et de fractions rationnelles. Acta Math. t. 24 (I901) p. 309-382. [14, 25, 28].

(27) Addition au mémoire sur les séries de polynomes et de fractions rationnelles. Acta Math. t. 24 (I90I) p. $3^{8} 3-3^{8} 7$. [14].

(28) Leçons sur les séries divergentes. Gauthier-Villars, Paris, I 901 . VII +182 p., $8^{\circ}$. [25, 31, 34].

(29) Remarques relatives à la communication de M. Mittag.Leffler. Compte Rendu du deuxième Congrès International des Mathématiçiens p. 277 278. Paris, Gauthier-Villars, I902. [25].

(30) Sur la généralisation du prolongement analytique (Voir Painlevé n:0 I3). Comptes Rendus, t. I35 (2 I Juillet I 902) p. I 50-I52. [28].

(3 $\mathrm{r})$ Leçons sur les séries à termes positifs. Paris, Gauthier-Villars I902. VI 93 p., $8^{\circ}$. [22]. 
(32) Sur la détermination de classes singulières de séries de Taylor. Comptes Rendus, t. I37 (2 Novembre I 903) p. 695-697. [23].

(33) Leçons sur les fonctions méromorphes. Paris, Gauthier-Villars, I 903 , VII +122 p., $8^{\circ}$. [1 $\left.3, \mathrm{I}_{4}, 24\right]$.

(34) Leçons sur les fonctions de variables réelles et les développements en séries de polynomes.

Paris, Gauthier-Villars (I 905), VIII + I 58 p., $8^{\circ}$. [ I 4,25$]$.

(35) La classification des ensembles de mesure nulle et la théorie des fonctions monogènes uniformes.

Comptes Rendus, t. I 54 (26 Février I9I2) p. 568-570. [16].

(36) Les séries de fonctions analytiques et les fonctions quasi-analytiques. Comptes Rendus, t. I54 (3 Juin I9 I 2) p. I 49 I-I 493. [I6].

(37) Sur la théorie du potentiel logarithmique.

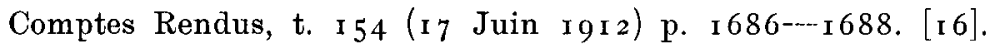

(38) Les fonctions monogènes non analytiques. Bulletin Soc. Math. de France, t. 40 (I9I2) p. 205-2 I9. [I6].

(39) Définition et domaine d'existence des fonctions monogènes uniformes. Proceedings of the Fifth International Congress of Mathematicians. Vol. I p. I33- I 44. Cambridge, University Press, I9 3. [r6].

(40) Monogenic uniform non-analytic functions.

The Rice Institute Pamplet, t. 4 n:o I (Janvier rgI7) p. 22-52. [r6].

(4 I) Leçons sur les fonctions monogènes uniformes d'une variable complexe. Paris, Gauthier-Villars, I 9 I 7, XII + I 65 p., $8^{\circ}$. [ [ I 2, I 3, I 4, I6, 28 ].

(42) Remarques sur la Note de M. J. Wolff. (Voir Wolff n:o r.) Comptes Rendus, t. I 73 (28 Novembre I921) p. I057-1059. [13, I4, I6].

(43) Sur la méthode de sommation exponentielle des séries divergentes. Matematisk Tidskrift, B, I 924, p. $40-4$ I. [3I].

\section{E. Bortolotti.}

(I) Sul raggio di convergenza della serie di potenze.

Mem. R. Accad, in Modena, 3:e série, t. 4 (I90I) p. I 4 -I6. [21].

\section{R. Braitseff.}

(I) Izyskanie osobych toček funkcij, opreděljaemoj rjadom Taylor'a. [= Recherche des points singuliers d'une fonction définie par une série de Taylor.]

Mat. Sbornik izd. Moskovskim Mat. Obšcestvom, t. 26 (1906-07) p. 242-3 I 4, 3 I 5-482, avec une addition au p. 544. [24].

(2) Nouvelle méthode de recherche des points singuliers d'une fonction définie par une série de Taylor (en russe).

Annales de l'Inst. Polytechnique de Varsovie, I9 Io, I50 p. Aussi publié séparément, $\mathrm{XXX}+\mathrm{I}_{5} \mathrm{I}$ p. [24]. 
Essai d'une bibliographie de la représentation analytique d'une fonction monogène. 15

(3) Sur les séries de Taylor dont les points singuliers sont situés sur l'axe des va. leurs réelles d'argument (en russe).

Annales de l'Inst. Polytechnique de Varsovie, Ig I r, 2 I p. [24].

(4) Addition au mémoire: Nouvelle méthode de recherche des points singuliers d'une fonction définie par une série de Taylor (en russe). Annales de I'Inst. Polytechnique de Varsovie, igi I, 2 I p., I9I 2, 8 p. [24].

(5) Sur les points singuliers d'une fonction définie par une série de Taylor (en russe). Annales de l'Inst. Polytechnique dé Varsovie, I9I3, 204 p. [24].

\section{H. Bremekamp.}

(I) Functies, die slechts in een bepaald deel van het complexe vlak bestaan. [=.Sur les fonctions existant seulement dans une partie restrainte du plan complexe.] Handel. Nederl. Natuur-en Geneesk. Congr. I3 (I9I1) p. I52-I6I. [I2].

\section{T. J. I'a. Bromwich.}

(I) Various extensions of Abel's lemma. Proceedings London Math. Soc., z:e série; t. 6 (I908) p. 58-76. [22].

(2) On the limits of certain infinite series and integrals. Math. Annalen t. 65 (I908) p. 350--369. [22, 31].

(3) An introduction to the theory of infinite series. London and New York, MacMillan, I go8, XIV + 5 I I p., $8^{\circ}$. [30]. [La 2:e éd. de I 926 ne traite pas la théorie de sommabilité.]

\section{F. Bucca.}

( ) Sullo sviluppo d'una funzione uniforme di variabile complessa, dotata di singularità isolate, in serie colle caratteristiche separate.

Rendiconti Cir. Mat. di Palermo, t. i I (1897) p. 90-Io3. [13, I4].

(2) Sopra certi integrali e certi sviluppi in serie.

Rendiconti Cir. Mat. di Palermo, t. I 2 (r898) p. 260-277. [I4].

\section{A. Buhl.}

(I) Sur une extension de la méthode de sommation de M. Borel. Comptes Rendus, t. r44 (2 Avril r907) p. 710-712. $[25,3$ r].

(2) Sur la sommabilité des séries de Laurent. Comptes Rendus, t. I 45 (1 4 Oct. I907) p. 6r4-6r7. $[25,3$ r]

(3) Sur de nouvelles applications de la théorie des résidus. Bull. des Sciences Math., t. 3I (1907) p. I 52-I58. [25, 3 I].

(4) Sur de nouvelles formules de sommabilité. Bull. des Sciences Math., t. 3 I (I907) p. $340-346$. [25, 31$]$.

(5) Sur les séries de polynomes tayloriens. Comptes Rendus, t. $1_{4} 6$ (16 Mars I908) p. $575^{--} 578$. [13, I 4,25 ]. 
(6) Sur la représentation des fonctions méromorphes par des séries de polynomes tayloriens.

Bull. des Sciences Math., t. 32 (1908) p. I98--207. [1 3, I 4, 25 ].

(7) Sur la sommabilité des séries d'une variable réelle ou complexe. Journal de Math., 6:e série, t. 4 (1908) p. 367-377. [25, $3 \mathrm{I}]$.

(8) Sur la transformation des séries asymptotiques en séries de polynomes tayloriens convergentes.

Comptes Rendus, t. I 50 (I 3 Juin I 9 Io) p. I 583 - I 585 . [ I 4, 25, 34].

(9) Sur la transformation de séries asymptotiques en séries de polynomes tayloriens. Annales de Toulouse, série 3, t. 2 (1910) p. 457-475. [14, 25, 34].

(10) Sur la représentation des fonctions méromorphes. Acta Math., t. 35 (l912) p. 73-95. [13, 14, 25, 31 ].

(I I) Sur les séries de polynomes tayloriens franchissant les domaines W. Comptes Rendus, t. I66 (2 I Mai I9 I8) p. 808-8 I1. [14, I6, 28].

(1 2) Sommabilité et fonction $E_{\alpha}(x)$. L'Enseignement mathématique, t. 24 (1924) p. 69-76. $[25,32]$.

( 13$)$ Séries analytiques. Sommabilité.

Mémorial des Sciences Mathématiques. Fasc. VII. Paris, Gauthier-Villars, I 925 , 54 p., $8^{\circ}$. [ r $\left.4,25,31,32\right]$.

\section{B. Bukrejeff.}

(I) Analitičeskija vyraženija odnoznačnych funkcij. [= Expressions analytiques des fonctions uniformes.]

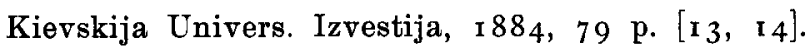

(2) O někotorych priloženijach teoremy g. Mittag-Lefflera. [= Applications du théorème de Mittag-Leffler.]

Kierskija Univers. Izvestija, I 885, p. $225-232$. [I 3].

(3) O razloženii transcendentnych funkcij na častnyja drobi. [= Sur la résolution en fractions partielles des fonctions transcendentes.]

Kievskija Univers. Izvestija, I886, n:os Io-1 2, 94 p. [I3].

\section{H. Burkhardt.}

(1) Über den Gebrauch divergenter Reihen in der Zeit von 1750-I860. Math. Annalen, t. 70 (I 9 I I) p. I69-206. [34].

(2) Über Funktionen grosser Zahlen, insbesondere über die näherungsweise Bestimmung entfernter Glieder in den Reihenentwicklungen der Theorie der Keplerschen. Bewegung.

Münchener Sitzungsberichte, I9 4, p. I-I I. [35].

\section{A. Caldarera.}

(I) Su talune estenzioni dei criteri di convergenza di Hardy e Landau. Note e memoria mat., Catania, t. 2 (1922) p. $77-98$. [33]. 
Essai d'une bibliographie de la représentation analytique d'une fonction monogène. 17

\section{T. Carleman.}

(I) Some theorems concerning the convergence of power series on the circle of convergence.

Arkiv f. Mat., Astr. o. Fys., t. I 5 N:o I I (192 I) I 3 p. [22].

(2) Sur les séries $\sum \frac{A_{v}}{z-\alpha_{v}}$.

Comptes Rendus, t. I 74 (27 Février I922) p. 588-59r. [13, I 4, I6].

(3) Sur les séries asymptotiques.

Comptes Rendus, t. I 74 (I 2 Juin I922) p. I $5^{27-r} 530$. [34].

(4) Sur le calcul effectif d'une fonction quasi analytique dont on donne les dérivées en un point. Comptes Rendus, t. I 76 (8 Janvier I923) p. 64-65. [28].

(5) Über die Approximation analytischer Funktionen durch lineare Aggregate von vorgegebenen Potenzen.

Arkiv f. Mat., Astr. o. Fys., t. 17 , n:o 9 (I923) 30 p. [I3, I 4$]$.

(6) Les fonctions quasi analytiques. Paris, Gauthier-Villars, I $926, I+116$ p., $8^{\circ}$. [16].

\section{F. Carlson.}

( I) Sur une classe de séries de Taylor. Thèse, Upsala, K. W. Appelberg, 19 I 4, 76p. [24].

(2) Une remarque sur la transformation de séries sommables en séries convergentes. Nyt Tidsskrift for Mat., B. t. 28 ( I $9 \mathbf{I}_{7}$ ) p. 81-88. [3 $\mathbf{r}$ ].

(3) Über Potenzreihen mit endlich vielen verschiedenen Koeffizienten.

Math. Annalen, t. 79. (I9I8) p. $237-245$. [24].

(4) Sur une propriété des polynomes d'une variable.

Comptes Rendus, t. 169 (29 Décembre I9 19) p. 1388-1390. [14, 23].

(5) Über Potenzreihen mit ganzzahligen Koeffizienten.

Math. Zeitschrift, t. $9($ I 92 I) p. I-I3. [23, 24$]$.

(6) Über ganzwertige Funktionen.

Math. Zeitschrift, t. I I (i 921$)$ p. I-23. [24].

(7) Sur le prolongement analytique.

Arkiv f. Mat., Astr. o. Fys., t. I 7 , n:o I I (1923) p. I-36. [25].

(8) Sur quelques suites de polynomes.

Comptes Rendus, t. I 78 (I9 Mai I924) p. I677-I680. [I4, 22].

(9) Sur la représentation des fonctions analytiques par des séries de polynomes.

Arkiv f. Mat., Astr. o. Fys., t. I 9 A, n:o 23 (1925) p. 1-7. [26].

\section{F. Carlson et E. Landau.}

(r) Neuer Beweis und Verallgemeinerungen des Fabryschen Lückensatzes. Göttinger Nachrichten, I 92 I, p. I $84-$ r 88 . [23].

\section{R. D. Carmichael.}

(I) General aspects of the theory of summable series. (Symposium lecture.)

Bulletin American Math. Soc., t. 25 (I9I8) p. 97-I3I. [30, 3 I].

3-2822. Acta mathematica. 52. Imprimé le 23 janvier 1929. 


\section{H. S. Carslaw.}

(1) Introduction to the theory of Fourier's series and integrals. London, MacMillan, I 92 I (2:e éd.) XI +323 p. $8^{\circ}$. [22, 3 I $]$.

\section{F. Casorati.}

(I) Aggiunte a recenti lavori dei sig:i Weierstrass e Mittag.Leffler sulle funzioni di una variabile complessa.

Annali di Mat., 2:e série, t. Io (I882) p. 26 I - 278. [I3].

(2) Sulle funzioni uniformi.

R. Ist. Lombardo. Rendiconti, z:e série, t. I 5 (I 882 ) p. $25^{1-2} 5^{2}$. [I3].

\section{E. Catalan.}

(I) Sur un théorème d'Abel.

Mathesis, t. 4 (1884) p. $25-28$. [22].

\section{A. L. Cauchy.}

(I) Cours d'Analyse de l'École Royale Polytechnique. ı:re partie. Analyse algébrique. Paris, Debure frères, I 82 I, XVI +576 p., $4{ }^{\circ}$.

Euvres, sér. 2, t. 3 Paris, Gauthier-Villars, I 897 , VIII +476 p. $4^{\circ}$. [2I].

(2) Résumés analytiques.

Turin, Imp. Roy. 1833, I66 p., $4^{\circ}$.

Euvres, sér. 2, t. Io. Paris, Gauthier-Villars, I 895, I 84 p., $4^{\circ}$. [21].

(I) Note on lacunary functions.

\section{A. Cayley.}

Quarterly Journal, t. 26 (I893) p. 279-281. [I 2].

\section{P. Cazzaniga.}

(I) Sopra una formola di Cauchy, concernante lo sviluppo di funzioni in prodotti infiniti.

Rendiconti R. Ist. Lombardo, z:e série, t. I5 (I882) p. 273-279. [13].

\section{N. Č ebotaref.}

(1) Sur la méthode de l'élimination des variables entre des équations transcendentes. Bulletin de la Soc. Phys.-math. de Kasan, série 2 (s), t. 24 (1924) p. I-6. [24].

\section{E. Cesàro.}

(I) Sur la multiplication des séries.

Bull. des Sciences Math., série 2, t. I4 (1890) p. I I 4-I 20 . [3 I].

(2) Sulla determinazione assintotica delle serie di potenze.

Rendiconto dell'Accad. fis. e mat. Napoli, 2 :e série, t. $7(1893)$ p. 187--195. [2z]. 
(3) Démonstration d'un théorème de M. Appell.

Mathesis, 2:e série, t. 3 (I 893 ) p. $24 \mathrm{I}-243$. [22].

\section{S. Chapman.}

(I) On the general theory of summability, with application to Fourier's and other series.

Quarterly Journal, t. 43 (I9II) p. 1-52. [30].

(2) On non-integral orders of summability of series and integrals.

Proceedings London Math. Soc., 2:e série, t. 9 (I gII) p. 369-409. [22, 3I].

\section{Cipolla.}

(I) Sul criterio di convergenza di Hardy.

Rendiconto Accad. sci. fis. e mat. di Napoli, série 3, t. 26 (1920) p. 96-107, I 5 - 160 . [33].

(2) Criteri di convergenza riducibili a quello di Hardy-Landau.

Rendiconto Accad. Sci. fis. e mat. di Napoli, 3:e série, t. 27 (I92 I) p. 28-37. [33].

\section{A. Costabel.}

(I) Sur le prolongement analytique d'une fonction méromorphe. L'enseignement math., t. Io (1908) p. $377-390$. [24, 25].

\section{P. Cousin.}

(1) Sur les fonctions d'une variable complexe admettant des singularités de nature quelconque.

Annales Fac. Sci. de Grenoble, t. 7 (1895) p. $225^{-2} 3^{8}$ [I3].

\section{P. Csillag.}

(I) A hatványsorra vonatkozó két összetartási kriterium kapósolatorol. [= Sur la con. nection entre deux critères pour la convergence de séries entières.]

Math. és phys. lapok, t. 26 (I9I7) p. 74-80. [22].

\section{E. Cunningham.}

(r) An extension of Borel's exponential method of summation of divergent series applied to linear differential equations.

Proc. London Math. Soc., (2) 3 (I904) p. I $57-169 .[27,3$ I].

\section{G. Darboux.}

(1) Mémoire sur l'approximation des fonctions de très-grands nombres, et sur une classe étendue de développements en série.

Journal de Math., série 3, t. 4 (I878) p. $5-5^{6}$ et $377-4$ I6. [I $4,22,24,35$. 


\section{W. Demuth.}

(I) Über unendliche Potenzreihen, deren Koeffizienten nach arithmetischen Reihen höherer Ordnung fortschreiten.

Programme n:o 857, Gymn. de Blankenburg am Harz, i 9o6, p. 3-20. [2 I].

\section{A. Denjoy.}

(I) Sur les fonctions analytiques uniformes qui restent continues sur un ensemble parfait discontinu de singularités.

Comptes Rendus, t. I48 (3 Mai I 909) p. I I 54-i I 56. [I3].

(2) Sur les fonctions analytiques uniformes à singularités discontinues.

Comptes Rendus, t. I 49 (26 Juillet I 909) p. 258-260. [I3].

(3) Sur les singularités discontinues des fonctions analytiques uniformes.

Comptes Rendus, t. I 49 (9 Août I 909) p. 386-388. [I3].

(4) Sur les fonctions analytiques uniformes à singularités discontinues non isolées.

Comptes Rendus, t. I 50 (3 Janvier I 9 Io) p. $3^{2-34}$. [I3].

(5) Sur une propriété des fonctions de variable complexe.

Comptes Rendus, t. I68 (24 Février I919) p. $387-389$. [12, 22 ].

(6) Sur les fonctions définies par des séries de fractions rationnelles. Comptes Rendus, t. I 74 (9 Janvier I 922 ) p. 95-98. [13, I4].

(7) Sur les séries de fractions rationnelles.

Comptes Rendus, t. I79（3 Novembre I924) p. $867-870$. [14].

(8) Sur les singularités des séries de fractions rationnelles.

Comptes Rendus, t. I 79 (ro Novembre I924) p. 958-960. [I 2, I 4, 28].

(9) Sur les séries de fractions rationnelles.

Bulletin Soc. Math. de France, t. 52 (1924) p. 418-434. [I4].

\section{Desaint.}

(I) Sur la représentation générale des fonctions analytiques quelconques. ${ }^{1}$

Comptes Rendus, t. 130 (9 Avril i 900) p. 999-I002. [I3].

(2) Sur les séries de Taylor et les étoiles correspondantes. Comptes Rendus, t. I 32 (6 Mai I 90I) p. I I 02 -I I05. [24, 25$]$.

(3) Théorèmes généraux sur les points singuliers des fonctions données par une série de Taylor.

Journal de Math. pures et appl,, série 5, t. 8 (1902) p. 433-45 I. [24].

(4) Sur le problème de la transformation dans les séries de Taylor.

Comptes Rendus, t. I 36 (I 5 Juin I 903) p. I $423-$ I $_{4} 25$. [24].

(5) Les séries de Taylor et la représentation exponentielle.

Annales de l'École Norm., série 3, t. 2 I (1904) p. 4 I5-448. [24].

(6) Sur les représentations générales des fonctions.

Comptes Rendus, t. I48 (24 Mai I909) p. 1376-I378. [I3].

${ }^{1}$ Le nom de l'auteur est épelé Desaints. Faute d'impression? 


\section{P. Dienes.}

(1) La série de Taylor sur le cercle de convergence. Comptes Rendus, t. I 40 (20 Février 1905) p. 489-49I. [22].

(2) Adalékok az analitikai függvények elméletéhez. [ $=$ Contributions à la théorie des fonctions analytiques.]

Math. és Phys. Lapok, t. I4 (1905) p. I6I-I92. [22].

(3) Sur les singularités des fonctions analytiques.

Comptes Rendus, t. I47 (2 r Déc. I 908) p. I 388 - I 390. [22, 31].

(4) Analitikai függvény viselkedés az összetartási körön. [= Les propriétés des fonctions analytiques sur le cercle de convergence.]

Math. és Phys. Lapok, t. I 8 (I909) p. I-i6. [22].

(5) Analitikai függvények végtelenségi helyeinek vizsgálata. [= Étude des points d'infinitude des fonctions analytiques.]

Math. és Phys. lapok, t. i 8 (1909) p. I $7-28$. [24].

(6) Sur les singularités des fonctions analytiques en dehors du cercle de convergence. Comptes Rendus, t. I 48 (I 5 Mars i 909) p. 694-698. [26, 3 x].

(7) Essai sur les singularités des fonctions analytiques.

Journal de Math., série 6, t. 5 (I909) p. 327-4 I3. Publié aussi comme Thèse, Paris, Gauthier-Villars, I909. 9I p. $4^{\circ}$. $[22,24,26]$.

(8) Sur les singularités d'ordre négatif des fonctions analytiques (hongrois).

Math. és termész. ért., t. 27 (1909) p. 58-63. [26].

(9) Sur un problème d'Abel.

Comptes Rendus, t. I5I (25 Juillet I9 I0) p. 294-296. [26].

(ro) Sur les séries de polynomes et les singularités des fonctions analytiques.

Comptes Rendus, t. I 52 (I 3 Février I 9 I I) p. 362-364. [26].

(I I) Sur la sommabilité de la série de Taylor.

Comptes Rendus, t. 153 (30 Octobre r9I1) p. $802-805 .[22,33]$.

(12) Leçons sur les singularités des fonctions analytiques.

Paris, Gauthier-Villars, I $9 I_{3}$. VII $+I_{7} 2$ p. $8^{\circ}$. $[22,25,26]$.

\section{P. et V. Dienes}

(I) Sur les singularités algébrico-logarithmiques.

Comptes Rendus, t. I49 (29 Novembre I909) p. 972-974. [26].

(2) Recherches nouvelles sur les singularités des fonctions analytiques.

Annales de l'École Norm., série 3, t. 28 (I9II) p. 389-457. [22, 24, 25, 26, 3I].

(3) Analitikai függvények algebrai és logaritmikus szingularitásairol. [ = Sur les sin. gularités algébriques et logarithmiques des fonctions analytiques.]

Math. és phys. lapok, t. 20 (I9II) p. $73-97,169-$ I97, et t. 2 I (I9I2) p. I24. $[24]$. 


\section{Dienes}

(I) Sur les points critiques logarithmiques.

Comptes Rendus, t. I 48 (26 Avril r 909) p. 1087-1090. [22, 26].

\section{R. Dietrich.}

(ז) Eine Untersuchung über Convergenzbezirke. (Voir G. Mittag-Leffler, n:o 30.) Archiv d. Math. u. Physik, t. 69 (1883) p. $38 \mathrm{r}-384$. [25].

\section{U. Dini.}

(r) Alcuni teoremi sulle funzioni di una variabile complessa.

In mem. Dominici Chelini. Collectanea mathematica. Milan, Hoepli, I88 I p. $25^{8}$ -276 . [1 3 ].

\section{G. Doetsch.}

(r) Eine neue Verallgemeinerung der Borelschen Summabilitätstheorie der divergenten Reihen. Inauguraldissertation, Göttingen I $920,5^{8}$ p., $8^{\circ} .[25,31,33]$.

(2) Über die Summabilität von Potenzreihen auf dem Rande des Borelschen Summa. bilitätspolygons.

Math. Annalen, t. 84 (192I) p. 245-25 I. $[26,3$ I $]$.

(3) Über die Cesàrosche Summabilität bei Reihen und eine Erweiterung des Grenz. wertbegriffs bei integrablen Funktionen.

Math. Zeitschrift, t. I I (I92I) p. 16I-I79. [3I, 33].

\section{G. Eisenstein.}

(1) Über eine allgemeine Eigenschaft der Reihen-Entwicklungen aller algebraischen Funktionen.

Verhandlungen d. K. Preuss. Akad. d. Wissenschatten zu Berlin, I852 p. 44I443. [24].

\section{A. C. Elsbach.}

(1) Over singuliere punten van door machtreeksen bepaalde functies. [= Sur les points singuliers de fonctions définies par des séries entières.]

Thèse, Leiden, E. Ydo, I 919,97 p., $8^{\circ}$. [22, 23, 24$]$.

\section{G. Faber.}

(I) Über Reihenentwicklungen analytischer Funktionen. Inauguraldissertation, München, I 903, $67 \mathrm{p} ., 8^{\circ}$. [ [ 3, I 4, 24, 25 ].

(2) Über die Fortsetzbarkeit gewisser Taylorscher Reihen. Math. Annalen, t. $57(19 \circ 3)$ p. 369-388. [24, 25$]$.

(3) Über polynomische Entwickelungen.

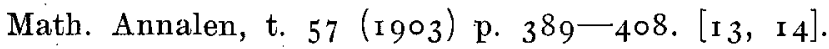


Essai d'une bibliographie de la représentation analytique d'une fonction monogène.

(4) Über die Nicht-Fortsetzbarkeit gewisser Potenzreihen. Sitzungsberichte ..... München, t. 34 (1904) p. 63-74. [23].

(5) Über analytische Funktionen mit vorgeschriebenen Singularitäten. Math. Annalen, t. 6o (I 905) p. 379-397. [I3].

(6) Über Potenzreihen mit unendlich vielen verschwindenden Koeffizienten. Sitzungsberichte ...... München, t. 36 (1906) p. 581-583. [24].

(7) Über polynomische Entwicklungen II. Math. Annalen, t. 64 (1907) p. I I6-I 35 . [1 3, I 4, 24$]$.

(8) Über Reihen nach Legendreschen Polynomen.

Jahresbericht d. deutschen Math.-Verein., t. I6 (1907) p. I09-1 I5. [13, 14, 24].

(9) Bemerkungen zu einem funktionentheoretischen Satze des Herrn Hadamard. Jahresbericht d. deutschen Math.-Verein., t. I6 (I907) p. 285-298. [24].

( 1 o) Beitrag zur Theorie der ganzen Funktionen.

Math. Annalen, t. 70 (I9I I) p. 48-68. [1 4, 24].

(I I) Über die Hölderschen und Cesàroschen Grenzwerte. Münchener Sitzungsberichte, I9I3 p. 5I9-53 I. [3I].

(1 2) Über das Verhalten analytischer Funktionen an Verzweigungsstellen. Münchener Sitzungsberichte, I9I7 p. $263-284$. [22, 24$]$.

(13) Über Tschebyscheffische Polynome. Journal f. Math., t. I 50 (I919-20) p. 79-106. [13, I4, I 5].

(14) Über nach Polynomen fortschreitende Reihen. Münchener Sitzungsberichte, I 922 p. I 57 - I 78 . [I 3, I4].

(15) Abschätzung von Funktionen grosser Zahlen. Münchener Sitzungsberichte, I 922 p. $285-304$. [22, 24, 35].

\section{E. Fabry.}

(r) Sur les points singuliers d'une fonction donnée par son développement en série et l'impossibilité du prolongement analytique dans des cas très généraux. Annales de l'École Norm., série 3, t. I 3 (1896) p. 367-399. [23, 24].

(2) Sur les séries de Taylor. Comptes Rendus, t. I 24 (1 8 Janvier I 897) p. I 42-I 43. [23].

(3) Sur les séries de Taylor. Comptes Rendus, t. I25 (20 Déc. I 897) p. I 086-1089. [22, 24$]$.

(4) Sur les points singuliers d'une série de Taylor. Journal de Math. pures et appl., série 5, t. 4 (1898) p. 317-358. [24].

(5) Généralisation du prolongement analytique d'une fonction. Comptes Rendus, t. 128 (9 Janvier r 899) p. $78-8$ o. [28].

(6) Sur les séries de Taylor qui ont une infinité de points singuliers. Acta Math., t. 22 (1899) p. 65-87. [23].

(7) Sur la série de Taylor et ses points singuliers. Nouvelles Annales de Math., série 4, t. 6 (1906) p. 503-507. [22]. 
(8) Les points singuliers et le prolongement.

Comptes Rendus du Congrès des Soc. savantes en r907, Sciences, Paris, Imp. Nat. I 908,2 p. $[23,24]$.

(9) Ordre d'une série de Taylor. Comptes Rendus, t. I 49 (6 Déc. 1909) p. I 043- г 045 . [22].

( 10$)$ Ordre des points singuliers d'une série de Taylor. Comptes Rendus, t. I 5 I (2 I Novembre rgro) p. 922-925. [22].

(1 I) Ordre des points singuliers de la série de Taylor.

Acta Math., t. 36 (I912) p. 69-104. [2 2].

\section{P. Fatou.}

(I) Sur les séries entières à coefficients entiers. Comptes Rendus, t. 138 (8 Février r904) p. 342-344). [24].

(2) La série de Fourier et la série de Taylor sur son cercle de convergence. Comptes Rendus, t. I39 (2 I Novembre r 904) p. 850-852. [2 2].

(3) Sur l'intégrale de Poisson et les lignes singulières des fonctions analytiques. Comptes Rendus, t. I 40 (6 Février 1905) p. 359-360. [22].

(4) Sur quelques théorèmes de Riemann. Comptes Rendus, t. I 40 (27 Février I 905) p. 569-570. [22].

(5) Séries trigonométriques et séries de Taylor. Acta Math., t. 30 (1906) p. 335-400. [22].

(6) Sur une classe remarquable de séries de Taylor. Annales de l'École Norm., série 3, t. 27 (1910) p. 43-53. [24].

(7) Sur les lignes singulières des fonctions analytiques. Bulletin Soc. Math. de France, t. 4I (I9I3) p. II3-1 I9. [1 2, 26].

(8) Sur les lignes singulières des fonctions analytiques. Comptes Rendus, t. i68 (10 Mars 1919) p. 501-502. [12, 22$]$.

(9) Sur l'évanouissement d'une branche de fonction uniforme aux points d'une ligne singulière.

Bulletin Sci. Math. série 2, t. 45 (192 I) p. 65-8I. [1 2, 22].

( 10$)$ Sur les fonctions holomorphes et bornées à l'intérieur d'un cercle. Bulletin Soc. Math. de France, t. 5I (1923) p. r9I-202. [22].

\section{Fejér.}

(r) Néhány tétel a hatvány sorról. [= Quelques théorèmes sur les séries entières.] Math. és Phys. Lapok, t. 9 (I900) p. 403-4IO. [2I, 23$]$.

(2) Untersuchungen über Fouriersche Reihen.

Math. Annalen, t. $5^{8}$ (I904) p. 5I-69. [2 2, 3I].

(3) Sur une méthode de M. Darboux.

Comptes Rendus, t. I 47 (30 Nov. I 908) p. ro40-I042. [22, 24, 35]. 
(4) Asymptotikus értékek meghatározásáról. [= Sur la détermination des valeurs asymptotiques.]

Math. és termész. ért., t. 27 (1909) p. I-33. [2 2, 24, 35].

(5) Über gewisse Potenzreihen an der Konvergenzgrenze.

Münchener Berichte, $3^{\mathrm{e}} \mathrm{mém.,}$ (I9Io), I 7 p. [22].

(6) Sur les singularités de la série de Fourier des fonctions continues.

Annales de l'École Norm., série 3, t. 28 (IgII) p. 63-io4. [22].

(7) Eine Bemerkung zur Mittag-Lefflerschen Approximation einer beliebigen analytischen Funktion innerhalb des Sterngebietes.

Acta Math., t. 35 (1912) p. 67-7 r. [25].

(8) La convergence sur son cercle de convergence d'une série de puissances effectuant une représentation conforme du cercle sur le plan simple. Comptes Rendus, t. I56 (6 Janvier I9 13 ) p. 48-49. [24].

(9) Über die Konvergenz der Potenzreihe an der Konvergenzgrenze in Fällen der konformen Abbildung auf die schlichte Ebene.

Schwarz' Festschrift I9I4, p. 42-53. [22].

(1 o) Über konjugierte trigonometrischen Reihen.

Journal f. Math., t. I 44 (r9r4) p. $48-56$. [22].

(I I) Fourierreihe und Potenzreihe.

Monatshefte für Math. u. Physik, t. 28 (I917) p. 64-76. [22].

(12) Über Potenzreihen, deren Summe im abgeschlossenen Konvergenzkreise iiberall stetig ist.

Münchener Berichte I 9 I 7 p. $33-50$. [22].

(13) Interpolation und konforme Abbildung.

Göttinger Nachrichten I9 8 p. 3 I $9-33$ I. [I 3, I4].

\section{Fekete.}

(I) Hatványsorok összegezhetöségének szükséges és elegendö feltételeiröl. [= Conditions nécessaires et suffisantes pour la sommabilité des séries entières.] Math. és Phys. Lapok, t. I9 (1910) p. 387-39ז. $[22,3 \mathrm{r}]$.

(2) A széttartó végtelen sorok elméletéhez. [= Sur la théorie des séries divergentes.] Math. és termész. ért., t. 29 (I9II) p. 7 I $9-726$. [3I].

(3) Vizsgálatok az absolut summabilis sorokról, alkalmazással a Dirichlet- és Fouriersorokra. [= Recherches sur les séries absolument sommable, avec applications aux séries de Dirichlet et de Fourier.]

Math. és termész. ért., t. 32 ( $19 \mathrm{I}_{4}$ ) p. 389-425. [3I].

(4) Über Potenzreihen, deren Koeffizienten fast alle ganzzahlig sind.

Math. Annalen, t. $96($ I926) p. 4ro-4I 7. [23, 24$]$.

4-2822. Acta mathematica. 52. Imprimé le 23 janvier 1929. 


\section{A. Féraud.}

(I) Sur la valeur approchée des coefficients d'ordre élevé dans les développements en séries.

Thèse, Paris, Gauthier-Villars, I897, 204. p., 4․ [22, 24, 35].

\section{J. B. Flamme.}

I) Recherches des expressions approchées des termes très éloignés dans les développements du mouvement elliptique des planètes.

Thèse, Paris, Gauthier-Villars, I 887 , I I 9 p., $4^{\circ}$. [35].

\section{W. B. Ford.}

(I) Sur la fonction définie par une série de Maclaurin.

Journal de Math. pures et appl., série 5, t. 9 (1903) p. $223-232$. [24].

(2) A set of criteria for the summability of divergent series.

Bulletin Amer. Math. Soc., t. I5 (I909) p. 439-444. [3 I].

(3) On the relation between the sum-formulas of Hölder and Cesàro.

American Journal of Math., t. 32 (I9Io) p. 315-326. [3I].

(4) A theorem on the analytic extension of power series.

Bulletin Amer. Math. Soc., série 2, t. I6 (1910) p. 507-510. [24, 25].

(5) Studies on divergent series and summability.

Univ. of Michigan Sci. Ser., t. 2, New York, Macmillan, I9 16, XI + I 94 p. [3 I, 34].

(6) A conspectus of the modern theory of divergent series.

Bulletin Amer. Math. Soc., t. 25 (I918) p. I-I 5. [30, 3r, 34].

\section{J. Franel.}

(I) Sur la théorie des séries.

Math. Annalen, t. 52 (1899) p. 529-549. [1 2, 22, 23$]$.

Ph. Franklin.

(I) Functions of a complex variable with assigned derivatives at an infinite number of points, and an analogue of Mittag-Leffler's theorem.

Acta Math., t. $47\left(\mathrm{I}_{92} 6\right)$ p. $37 \mathrm{I}-385$. [1 3 ].

\section{Fréchet.}

( 1 ) Les fonctions prolongeables.

Comptes Rendus, t. I65 (I 2 Novembre I917) p. 669-670. [23].

\section{Fredholm.}

(r) Om en speciell klass af singulära linier. [= Sur une classe spéciale de lignes singulières.]

Öfversigt K. Vetenskaps-Akad. Förhandl. t. 47 (1890) p. I $3 \mathrm{I}^{-1} 34$. [1 2, 23$]$. 
(2) Sur la méthode de prolongement analytique de M. Mittag-Leffler.

Öfversigt K. Vetenskapsakademiens Förhandl. t. $5^{8}$ (I90I) p. 203-205. [25].

\section{Frenzel.}

(I) Die Darstellung der eindeutigen analytischen Functionen durch unendliche Producte und Partialbruchreihen.

Zeitschrift für Math, u. Physik, t. 24 (I879) p. 316--343. [I3, I4].

\section{G. Frobenius.}

(I) Über die Leibnitzsche Reihe.

Journal für Math. t. 89 (1880) p. 262-264. [22, 3I].

M. Fujiwara.

(r) Über summierbare Reihen und Integrale.

Tôhuku Math. Journal, t. I5 (I9I9) p. 323-329. [33].

(2) Ein Satz über die Borelsche Summation.

Tôhuku Math. Journal, t. I 7 (I920) p. 339-343. [3 I, 33].

\section{B. Gavrilovitch.}

( 1 ) Sur l'expression analytique de certaines fonctions (en serbe).

Mém. Acad. de Belgrade, t. 6I (I 900) p. 55-68. [I3?].

\section{G. A. Gibson.}

(I) An extension of Abel's theorem on the continuity of a powerseries. Proceedings Edinburgh Math. Soc., t. I9 (I901) p. 67-70. [22].

\section{J. Gillet.}

(1) Sur les fonctions à espaces lacunaires.

El progreso matemático, t. $3($ I 893$)$ p. I 39-I 4 I. [ [3].

\section{W. Goloubeff.}

(I) Sur les fonctions à singularités discontinues. Comptes Rendus, t. I58 (I8 Mai I9I4) p. I407-I408. [I3].

(2) O svězdĕ Mittag-Leffler'a. [= Sur l'étoile de M. Mittag-Leffler.]

Mat. Sbornik izd. Moskovskim Mat. Obšcestvom, t. 29 (I9I3-I 5) p. I 7 I-I 8 I. [25].

\section{F. Gomes Teixeira.}

(I) Exemples de fonctions à espaces lacunaires.

Nouvelles Annales de Math., 3:e série, t. 6 (1887) p. 43-44. [1 2].

(2) Extrait d'une lettre de M. Gomes Teixeira à M. Jules Tannery.

Bulletin Sciences Math., 2:e série, t. I I (I887) p. I93-I94. [I2]. 
Einar Hille.

(3) Extrait d'une lettre adressée à M. Hermite. Bulletin Sciences Math., série 2, t. I7 (I893) p. 29-32. [I2].

(4) Sobre o desenvolvimento das funcções em série.

Memoria premiada e publicada pela R. Acad. de sciencias ... de Madrid.

Memorias de la $R$. Acad. de Ciencias exactas, fisicas y naturales de Madrid, t. I 8 (I897) i r6 p. Obras t. I (I904) p. I-102. [r3, r4, 2 I].

(5) Sur les séries ordonnées suivant les puissances d'une fonction donnée.

Journal für Math., t. 122 (1900) p. 97-I23. [I 4].

(6) Sur la convergence des formules d'interpolation de Lagrange, de Gauss etc.

Journal für Math., t. I 26 (I903) p. I I 6-I62. [I 4 , I 5].

\section{E. Goursat.}

(1) Sur les fonctions uniformes présentant des lacunes. Comptes Rendus, t. 94 ( 13 Mars 1882) p. 7 I5-718. [12].

(2) Sur la théorie des fonctions uniformes. Comptes Rendus, t. 96 (26 Février r 883) p. 565-568. [r3].

(3) Sur les fonctions à espaces lacunaires. Bulletin Sci. Math., série 2, t. I I (I887) p. Io9-I I4. [I 2].

(4) Sur une fonction à espace lacunaire. Bulletin Sci. Math., série 2, t. I 7 (1893) p. 247-248. [1 2].

(5) Sur le prolongement analytique.

Comptes Rendus, t. I 28 (6 Mars I 899) p. 59I-593. [I I, 2 I ].

(6) Sur quelques développement de $\frac{I}{I-x}$ en séries de polynomes.

Bulletin Sci. Math., série 2, t. 27 (1903) p. 226-232. [25].

\section{M. Grisar.}

(1) Über eine Verallgemeinerung des Tauberschen Satzes und seine Ausdehnung auf $n$-fache Reihen.

Thèse, München, 1924, 59 p., $8^{\circ}$. [22, 23$]$.

\section{T. H. Gronwall.}

(I) Sur quelques méthodes de sommation et leur application à la série de Fourier. Comptes Rendus, t. I 58 (8 Juin I9I4) p. I664-I665. [25, 31].

(2) A problem in geometry connected with the analytic continuation of a power series. Annals of Math., série 2, t. I 8 (1916) p. 65-69. [2 ז].

(3) Über einige Summationsmethoden und ihre Anwendung auf die Fouriersche Reihe. Journal fiur Math., t. I 47 (1917) p. I6-35. [25, 3i $]$.

(4) Summation of series and conformal mapping.

Bulletin Amer. Math. Soc., t. 32 (I926) p. 36. $[22,25,31]$. 


\section{W. Gross.}

(1) Zur Poisson'schen Summierung.

Wiener Sitzungsberichte, t. I 24 , II a (I9I5) p. IOI 7-I037. [22, 3I].

\section{Guichard.}

(I) Théorie des points singuliers essentiels.

Annales de l'École Norm., série 2, t. I 2 (1883) p. 30I-394. [I3].

\section{A. Haar. ${ }^{1}$}

(I) Über analytische Funktionen mit singulärer Linie.

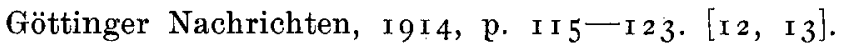

\section{J. Hadamard.}

(I) Sur le rayon de convergence des séries ordonnées suivant les puissances d'une variable.

Comptes Rendus, t. 106 (23 Janvier I888) p. 259-262. [2 I, 22].

(2) Sur la recherche des discontinuités polaires.

Comptes Rendus, t. 108 (8 Avril r 889) p. 722-724. [24].

(3) Essai sur l'étude des fonctions données par leur développement de Taylor.

Journal de Math., série 4, t. $8(\mathrm{r} 892)$ p. IoI-I 86. [2 2, 24$]$.

(4) Théorème sur les séries entières.

Comptes Rendus, t. 124 (8 Mars I 897) p. 492. [24].

(5) Sur les séries entières.

Procès-verbaux ... de Bordeaux. I $896-97$ p. I Io-I I 2. [24].

(6) Théorème sur les séries entières.

Acta Math., t. 22 (1899) p. 55-63. [24].

(7) La série de Taylor et son prolongement analytique.

Paris, C. Naud. Coll. Scientia, série phys.-math. n:o r 2. I $90 x$. VIII + 102 p., $8^{\circ}$. [20].

\section{J. Hadamard et $\mathbf{S}$. Mandelbrojt.}

(I) La série de Taylor et son prolongement analytique. Deuxième édition revue et mise au courant des progrès récents.

Paris, Gauthier-Villars. Coll. Scientia, série phys.-math. n:o 4 I, I :er volume I926. I $05 \mathrm{p}, 8^{\circ}$. [20].

\section{F. Hallgren.}

(I) Bidrag till teorien för entydiga funktioner. [ $=$ Contributions à la théorie des fonctions uniformes.]

Göteborg, Vet. o. Vitt. Samh. Handl. 2:e série, t. $3^{2}(\mathrm{r} 897)$ p. 55-8 I. [I 2, I 3,22$]$.

\footnotetext{
1 Voir p. 8 o.
} 


\section{Hamy.}

(I) Sur l'approximation des fonctions de grands nombres. Journal de Math., série 6, t. 4 (1908) p. 203-28r. [22, 35].

(2) Sur l'approximation des fonctions de grands nombres. Comptes Rendus, t. I 72 (29 Mars I92 I) p. $785-790$. [35].

(3) Sur l'approximation des fonctions de grands nombres, II. Mémoires de l'Acad. des Sciences, 2:e série, t. 57 (1922) p. 5-42. [35].

\section{Hanni.}

(I) Über Borel's Verallgemeinerung des Grenzbegriffes. Monatshefte für Math. und Physik, t. I2 (I90I) p. 265-289. [25, 3I].

(2) Zurückführung der allgemeinen Mittelbildung Borel's auf Mittag-Leffler's $n$-fach unendliche Reihen. Monatshefte für Math. und Physik, t. 14 (1903) p. 105-124. [25, 3I].

(3) Über die Beziehungen zwischen der Darstellung eines eindeutigen Zweiges einer monogenen Function durch Herrn Mittag-Leffler, der Methode der Mittelwerte des Herrn Borel und der Transformation des Herrn Lindelöf.

Acta Math., t. 29 (1905) p. 25-58. [25, 3r].

\section{Hansen.}

(I) Démonstration de l'impossibilité du prolongement analytique de la série de Lambert et des séries analogues.

Oversigt K. Danske Videnskab. Selskabs Forhandl., I907, n:o I, p. 3- I9. [12, 23 ].

(2) Recherches sur les singularités de certaines séries spéciales sur leur cercle de convergence.

Mémoires de l'Acad. R. de Danemark, 7:e série, t. 6 (I908) p. I-37. [1 2, 23$]$.

\section{G. H. Hardy.}

(I) On differentiation and integration of divergent series. Transactions Cambridge Phil. Soc. t. I9 (1903) p. 297-321. [3 I].

(2) Researches in the theory of divergent series and divergent integrals. Quarterly Journal, t. 35 (1904) p. 22-66. [30].

(3) A. method for determining the behaviour of certain classes of power series near a singular point of the circle of convergence. Proceedings London Math. Soc., z:e série, t. 3 (1905) p. 38I-389. [2 2].

(4) Some theorems connected with Abel's theorem on the continuity of power series. Proceedings London Math. Soc., série 2, t. 4 (1905) p. 247-265. [2 2].

(5) Some theorems concerning infinite series.

Math. Annalen, t. 64 (1907) p. $77-94 .[22,31]$. 
Essai d'une bibliographie de la représentation analytique d'une fonction monogène.

(6) On the singularities of functions defined by Taylor's series. (Remarks in addition to a former paper.)

Proceedings London Math. Soc., z:e série, t. 5 (1907) p. I97-205. [24].

(7) Generalisation of a theorem in the theory of divergent series. Proceedings London Math. Soc., z:e série, t. 6 (r908) p. 255-264. [31].

(8) The application to Dirichlet's series of Borel's exponential method of summation.

Proceedings London Math. Soc., (2) 8 (1909) p. 277-294. [27, 31].

(9) Theorems relating to the summability and convergence of slowly oscillating series.

Proceedings London Math. Soc., 2:e série, t. 8 (т910) p. 301-320. [33].

(1.) Notes on some points in the integral calculus. XXXI, The uniform convergence of Borel's integral.

Messenger of Math., série 2, t. 40 (I9II) p. I6I-r65. [3I].

( I I) An extension of a theorem on oscillating series. Proceedings London Math. Soc., (2) I2 (I912) p. I 74-I80. [33].

(I2) Notes on some points in the integral calculus. XXXVII. On the region of convergence of Borel's integral.

Messenger of Math., série 2, t. 43 ( 19 I $\left._{3}\right)$ p. 22-24. [25, 3I].

(13) Oscillating Dirichlet's integrals: An essay in the »Infinitärcalcül» of Paul du Bois-Reymond.

Quarterly Journal, t. 44 (x9r3) p. I-40. [35].

(x4) A theorem concerning Taylor's series.

Quarterly Journal, t. 44 (I9I3) p. 147-I60. [22].

(15) Note in addition to a paper on Taylor's series.

Quarterly Journal, t. 45 (r9I4) p. 77-84. [22].

(16) Note on Lambert's series.

Proceedings London Math. Soc., 2:e série, t. I3 (I914) p. I92- I98. [I2].

( 17 ) The second theorem of consistency for summable series.

Proceedings London Math. Soc., (2) I 5 (1915) p. 72-88. [31].

(18) Sur la sommation des séries de Dirichlet.

Comptes Rendus, t. I62 (27 Mars r9i6) p. 463-466. [3 I].

(I9) The application of Abel's method of summation to Dirichlet's series.

Quarterly Journal, t. 47 (I 9 I6) p. I 76 - 92 . [3 I].

(20) Notes on some points in the integral calculus. XLIII. On the asymptotic value of a definite integral, and the coefficients in a power series.

Messenger of Math., t. 46 (I9I6) p. 70-73. [22, 24, 35].

(2I) On two theorems of F. Carlson and S. Wigert. Acta Math., 42 (I919-20) p. 327-339. [24].

(2 2) A theorem concerning summable series.

Proceedings Cambridge Phil. Soc., t. 20 (x92 x) p. 304-307. [3I]. 


\section{G. H. Hardy et J. Chapman.}

(I) A general view of the theory of summable series. Quarterly Journal, t. 42 ( 19 r r) p. I 8 I-2 I 5. [30].

\section{G. H. Hardy et J. E. Littlewood.}

(I) The relations between Borel's and Cesàro's methods of summation.

Proceedings London Math. Soc., (2) II (I9II) I-I6. [22, 33].

(2) Contributions to the arithmetic theory of series.

Proceedings London Math. Soc., série 2, t. II (x9I2) p. 4II-478. [22, 33].

(3) Tauberian theorems concerning series of positive terms.

Messenger of Math., 2:e série, t. 42 (19r3) p. r9r-r92. [22, 33].

(4) Some theorems concerning Dirichlet's series.

Messenger of Math., (2) 43 (I9I4) p. I 34-I 47. [2 2, 31, 33].

(5) Some problems of diophantine approximation II.

Acta Math., t. 37 (1914) p. 193-238. [2 2].

(6) Tauberian theorems concerning power series and Dirichlet's series whose coefficients are positive.

Proceedings London Math. Soc., série 2, t. I3 (I9I4) p. I 74-I9I. [33].

(7) Theorems concerning the summation of series by Borel's exponential method. Rendiconti Circ. mat. di Palermo, t. 4 I (I9I6) p. 36-53. [3I, 33].

(8) Some problems of diophantine approximation: A remarkable trigonometrical series. Proceedings Nat. Acad. of Sci., Washington, t. 2 ( 1916 ) p. 583-586. [22, 23 ].

(9) Sur la convergence des séries de Fourier et des séries de Taylor. Comptes Rendus, t. I65 (24 Déc. I9 1 7) p. I047- го49. [2 2, 33].

(io) Abel's theorem and its converse.

Proceedings London Math. Soc., série 2, t. I 8 (r919-20) p. $205-235$ et t. 22 (r923) p. $254-269$. [22, 33].

( I ) Solution of the Cesàro summability problem for power-series and Fourier series. Math. Zeitschrift, t. I9 (1924) p. 67-96. [22, 3 ז, 33].

(12) A further note on the converse of Abel's theorem.

Proceedings London Math. Soc., série 2, t. 25 (1926) p. 219-236. [22, 33].

\section{G. H. Hardy et M. Riesz.}

(1) The general theory of Dirichlet's series.

Cambridge Math. Tracts n:o I 8 , I9I5, 78 p., $8^{\circ}$. [3 I].

\section{F. Hausdorff.}

(1) Zur Verteilung der fortsetzbaren Potenzreihen.

Math. Zeitschrift, t. 4 (1919) p. 98-103. [23].

(2) Summationsmethoden und Momentfolgen. I, II.

Math. Zeitschrift, t. 9 (I92I) p. 74-109 et 280-299. [22, 30, 33]. 
Essai d'une bibliographie de la représentation analytique d'une fonction monogène.

33

\section{T. Hayashi.}

(r) On Kronecker's example of power-series having its circle of convergence for its natural limit.

Tôhuku Math. Journal, t. 5 (I9 I 4) p. 6I-64. [23].

(2) Power-series having its circle of convergence for its natural limit.

Tôhuku Math. Journal, t. 6 (I9 I4-I 5) p. I43-I 45. [23].

\section{Æ. Heine.}

(I) Der Eisensteinsche Satz über Reihen-Entwickelung algebraischer Functionen. Journal für Math., t. 45 (1853) p. $285-302$. [24].

(2) Théorèmes d'Eisenstein sur les séries qui sont les développements de fonctions algébriques.

Nouvelles Annales de Math., t. I3 (I854) p. 245-253. [24].

\section{Ch. Hermite.}

(I) Sur quelques points de la théorie des fonctions. Extrait d'une lettre à M. Mittag. Leffler.

Journal für Math., t. 9 I (I $88_{\text {I }}$ ) p. $54-78$ et Acta Soc. Sci. Fennicae, t. I 2 ( $188_{3}$ ) p. $67-94 .[12,13]$.

(2) Sur une application du théorème de M. Mittag-Leffler, dans la théorie des fonctions. Extrait d'une lettre adressée à M. Mittag-Leffler.

Journal für Math., t. 92 (x882) p. I $45^{-1} 55$ et Acta Soc. Sci. Fennicae, t. I 2 (г 883 ) p. $425-436$. [r3].

(3) Cours de M. Hermite, professés pendant le $2: e$ semestre 1881-82. Rédigé par M. Andoyer.

Paris, A. Hermann, I 882,202 p., $4^{\circ}$. [I 2, I 3, 14, 2 I ].

\section{F. Hilb et M. Riesz.}

(I) Neuere Untersuchungen über trigonometrischen Reihen.

Encyklopädie der Math. Wissenschaften, t. II: 3, fasc. 8 (I924) p. I I $89-$ I 228 . $[22,3 \mathbf{I}]$.

\section{Đ. Hilb et O. Szász.}

(I) Allgemeine Reihenentwicklungen.

Encyklopädie der Math. Wissenschaften, t. II: 3, fasc. 8 (r924) p. r229-1276.

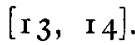

\section{Hilbert.}

(I) Ueber die Entwickelung einer beliebigen analytischen Function einer Variabeln in eine unendliche nach ganzen rationalen Functionen fortschreitende Reihe.

Göttinger Nachrichten, (1897) p. 63-70. [I $3 ; 14]$.

5-2822. Acta mathematica. 52. Imprimé le 24 janvier 1929. 


\section{J. M. Hill.}

(I) On a geometrical proposition connected with the continuation of power-series. Proceedings London Math. Soc., t. 35 (r902) p. 4I-5o. [2I].

(2) The continuations of certain fundamental power series.

Proceedings London Math. Soc. t. 35 ( 903$)$ p. $388-4$ I6. [2 I].

E. W. Hobson.

(I) The theory of functions of a real variable and the theory of Fourier's series. II. Cambridge, University Press, 1926 (2:e éd.) X+780 p., $8^{\circ}$. [22, 3I].

\section{O. Hölder.}

(I) Grenzwerthe von Reihen an der Convergenzgrenze.

Math. Annalen, t. $20($ I 882$)$ p. $535-549$. [22, 3I $]$.

\section{H. Holzberger.}

(1) Über das Verhalten von Potenzreihen mit zwei und drei Veränderlichen an der Konvergenzgrenze.

Monatshefte f. Math. u. Physik, t. 25 (I9I4) p. I79-266. Aussi comme thèse, München, I 9 I 3,90 p., $8^{\circ}$. [22].

\section{Th. Homén.}

(I) Analytisk framställning av några lakunära funktioner. [= Représentation analy. tique de quelques fonctions lacunaires.]

Acta Soc. Sci. Fennicae, t. I 2 (I $\left.88_{3}\right)$ p. $445-464$. [I 2$]$.

\section{G. Humbert.}

(т) Sur le développement d'une fonction suivant les puissances croissantes d'un po. lynôme.

Bulletin. Soc. Math. de France, t. 8 (I 880) p. I 24-I28. [I4].

\section{A. Hurwitz.}

(1) Über die Entwickelung der allgemeinen Theorie der analytischen Funktionen in neuerer Zeit.

Verhandlungen des I. Internationalen Mathematiker-Congresses in Zürich r897, Leipzig, B. G. Teubner, p. 9 I-I I 2. [10].

(2) Sur un théorème de M. Hadamard.

Comptes Rendus, t. I 28 (6 Février I 899) p. 350-353. [24].

\section{A. Hurwitz et G. Pólya.}

(r) Zwei Beweise eines von Herrn Fatou vermuteten Satzes.

Acta Math., t. 40 (I9I5) p. I $79-$ I 83 . [23]. 


\section{W. A. Hurwitz.}

(I) Report on topics in the theory of divergent series.

Bulletin Amer. Math. Soc., t. 28 (1922) p. I $7^{-3} 3^{6}$. [30].

(2) A trivial Tauberian theorem.

Bulletin Amer. Math. Soc., t. 32 (1926) p. $77-82$. [33].

\section{W. A. Hurwitz et L. L. Silverman.}

(I) On the consistency and equivalence of certain definitions of summability.

Transactions Amer. Math. Soc., t. I8 (I9I7) p. I-20. [3I, 32].

\section{A. F. Ingham.}

(I) Note on the converse of Abel's continuity theorem.

Procedings London Math. Soc., 2:e série, t. 23 (I924) p. 326-336. [22, 3x, 33].

F. H. Jackson.

(I) Borel's integral and $q$-series.

Proc. Roy. Soc. of Edinburgh, t. 30 (I910) p. $378-3^{8} 5$. [3 I].

C. G. J. Jacobi.

(r) Über Reihenentwickelungen, welche nach den Potenzen eines gegebenen Polynoms fortschreiten, und zu Coefficienten Polynome eines niederen Grades haben.

Journal fïr Math., t. $53\left(\mathbf{1}_{57}\right)$ p. Io3-126. Gesammelte Werke, t. 6, p. 203 -230. [I 4 ].

\section{E. Jacobsthal.}

(I) Mittelwertbildung und Reihentransformation.

Math. Zeitschrift, t. 6 ( 1920$)$ p. I00-I I 7 . [30].

\section{K. Jahraus.}

(I) Das Verhalten der Potenzreihen auf dem Konvergenzkreise.

Programme Gymn. Ludwigshafen a. Rh. I902, 56 p., $8^{\circ}$. [2.2].

\section{G. James.}

(I) Some theorems on the summation of divergent series.

Thèse, Columbia, New York, I9I 7,27 p. [3०, 3I].

(2) On the theory of summability.

Annals of Math., 2:e série, t. 2 I (I919) p. I20-127. [30].

\section{J. L. W. V. Jensen.}

(I) En nødvændig og tilstrækkelig Betingelse for at en given Taylor'sk Række ikke har singulære Punkter udenfor et retlinet Snit. [= Une condition nécessaire et 
suffisante pour qu'une série de Taylor donnée n'ait pas de points singuliers en dehors d'une coupure rectiligne.]

Nyt Tidsskrift for Mat. (B), t. 2 I (1910) p. 49-52. [24].

\section{R. Jentsch.}

(I) Untersuchungen zur Theorie der Folgen analytischer Funktionen.

Thèse, Berlin, I9I4, 39 p., $8^{\circ}$, et Acta Math., t. 4I (I9I7) p. 2 I9-25I. [I4, 22].

(2) Fortgesetzte Untersuchungen über die Abschnitte von Potenzreihen. Acta Math., t. 4 I (19I7) p. $253-270$. [23, 24$]$.

(3) Über Potenzreihen mit endlich vielen verschiedenen Koeffizienten.

Math. Annalen, t. 78 (I917) p. $276-285$. [24].

\section{F. Johansson.}

(r) Über die Nullstellen gewisser mit $E_{\alpha}(x)$ verwandten Funktionen.

Arkiv för Mat., Astr. o. Fys., t. i 8, n:o I I (1924) $5^{6}$ p. Thèse, Stockholm, I $924 \cdot[32]$.

(1) Note sur la série $\sum_{n=1}^{\infty} \frac{x^{n}}{n^{s}}$.

\section{A. Jonquière.}

Öfversigt K. Vetenskapsakad. Förhandl. t. 46 (1889) p. $257-268$ et Bull. Soc. Math. de France, t. I 7 (i 889 ) p. I 42 - I 52 . [24].

\section{G. Julia.}

(1) Sur les lignes singulières de certaines fonctions analytiques.

Bulletin Soc. Math. de France, t. 4I (I9I3) p. 35I-366. [I2].

(2) Les séries de fractions rationnelles et l'intégration. Comptes Rendus, t. I 74 (6 Février I922) p. 370-373. [r3, I4, I6].

(3) Séries de fractions rationnelles d'itération.

Comptes Rendus, t. I 80 (23 Février I925) p. 563-566. [I4, I6].

(4) Sur les séries de fonctions rationnelles itérées.

Comptes Rendus, t. I8 I (28 Déc. I925) p. I I I9-II22. [I2, I3, I4].

\section{S. Kakeya.}

(I) On some applications of a theorem about polynomial sequence.

Tôhuku Math. Journal, t. 5 (I9I4) p. 40-44. [I 4, 24].

\section{E. Kálmán.}

(I) Über die Abhängigkeit der Konvergenz einer Potenzreihe von der Konvergenz ihrer reellen oder imaginären Komponente.

Math. Annalen, t. 63 (1907) p. $322-325 \cdot[2 \mathrm{I}]$. 


\section{Kalmár.}

(г) Az interpolációról. [= Sur l'interpolation.]

Math. és Phys. Lapok, t. 33 (I926) p. I $20-$ I 49. [I3, I 4].

\section{J. Kampé de Fériet.}

( $)$ Sur l'uniformisation d'une classe de fonctions définies par des séries entières à coefficients méromorphes.

Comptes Rendus, t. I 82 (I I Janvier I 926 ) p. I I 3 -I I 4. [24, 25$]$.

\section{Karamata.}

(1) Sur certaines limites rattachées aux intégrales de Stieltjes. Comptes Rendus, t. I 82 (29 Mars I926) p. $833-835$. [24].

\section{A. Kienast.}

(I) Über die Darstellung der analytischen Funktionen durch Reihen, die nach $P_{0}$ tenzen eines Polynoms fortschreiten und Polynome eines niederen Grades zu Koeffizienten haben.

Dissertation, Zürich, 1906, 62 p., $8^{\circ}$. [ [ 13,14$]$.

(2) Über eine Integralformel und die Eigenschaften der darin vorkommenden Funktionen. Vierteljahrschrift d. Naturforschende Gesellschaft in Zürich, t. 6r (r9r6) p. 684 -725 . [13].

(3) Neue Entwicklungen über die Abel'sche Integralumkehrungsformel.

Vierteljahrschrift d. Naturf. Ges. in Zürich, t. 62 (I9I7) p. 59-66. [25].

(4) Sur quelques représentations arithmétiques des fonctions analytiques. L'Enseignement Math., t. I 9 ( I9I7) p. I 29-150. [25].

(5) Extensions of Abel's theorem and its converses. Proc. Cambridge Phil. Soc., t. I9 (I918) p. I29-147. [22, 31, 33].

(6) Erweiterungen des Abelschen Satzes für Potenzreihen und ihre Umkehrungen. Vierteljahrschrift d. Naturf. Ges. in Zürich, t. 67 (I922) p. 209-223. [2 2, 31, 33].

(7) Ueber eine Klasse von Grenzwertverfahren in der das arithmetisch-geometrische Mittel enthalten ist.

Vierteljahrschrift d. Naturf. Ges. in Zürich, t. 68 (1923) p. 228-290. [I3].

\section{G. A. Kinn.}

(I) Die Anwendung unendlicher Produkte in der Funktionentheorie. Programme Gymn. de Sachsisch-Regen (Transsylvanie), I899 p. 3-33. [13].

\section{J. C. Kluyver.}

(I) De formules van Borel over divergente reeksen. [ $=$ Les formules de $M$. Borel pour les séries divergentes.]

K. Akad. van Wetenschappen te Amsterdam. Verslag etc., t. 8 (I900) p. 33 I337. $[3 \mathrm{I}]$. 
(2) Over de ontwikkeling van eene functie in eene reeks van veeltermen. [= Sur le développement d'une fonction dans une série de polynomes.]

K. Akad. van Wetenschappen te Amsterdam. Verslag etc., t. 9 (IgoI) p. 608 -6 I 3 . [I 4,25$]$.

(3) Over veeltermreeksen. [ = Sur les séries de polynomes.]

K. Akad. van Wetenschappen te Amsterdam. Verslag etc., t. io (1902) p. 530 $-544,647-664$. [14, 25].

(4) Vraagstuk IX. Oplossing van Dr. J. H. M. Falkenhagen.

Wiskundige Opgaven, t. Io (I907) p. I $7-20$. [25].

(5) Over analytische functies, die door zekere reeksen van Lambert worden bepaald. [ - Sur les fonctions analytiques définies par certaines séries de Lambert.]

K. Akad. van Wetenschappen te Amsterdam. Verslag etc., t. 29 (I92 I) p. I I72 -II 80. [1 2, 14, 22, 27$]$.

\section{K. Knopp.}

(I) Grenzwerthe von Reihen bei der Annäherung an die Konvergenzgrenze.

Thèse, Berlin, I $907,5^{\circ}$ p., $8^{\circ}$. $[22,31]$.

(2) Eine notwendige und hinreichende Konvergenzbedingung.

Rendiconti Circ. Mat. di Palermo, t. 25 (1908) p. $237^{-2} 5^{2}$. [33].

(3) Über Lambertsche Reihen.

Journal für Math., t. I42 (I9I3) p. $283-3{ }^{1} 5$. Correction ib. t. I43 (I9I3) p. 50. [1 2,23$]$.

(4) Bemerkung zu der vorstehenden Arbeit des Herrn I. Schur (Voir I. Schur n:o r). Math. Annalen, t. 74 (I9I3) D. $459-46 \mathrm{r}$. [3 I].

(5) Mittelwertbildung und Reihentransformation. Math. Zeitschrift, t. 6 (1920) p. I 18--I23. [30].

(6) Über das Eulersche Summierungsverfahren.

Math. Zeitschrift, t. I $5(1922)$ p. $226-253$. $[25,3$ I $]$.

(7) Über das Eulersche Summierungsverfahren. (II. Mitteilung.)

Math. Zeitschrift, t. 18 (1923) p. I $25^{-1} 5^{6}$. [25, 26, 3 I, 33].

(8) Zur Theorie der $C$ - und $H$-Summierbarkeit.

Math. Zeitschrift, t. I9 (1923) p. 97--I I3. [3 I, 33].

(9) Neuere Untersuchungen in der Theorie der divergenten Reihen.

Jahresbericht d. Deutschen Math.-Vereinigung, t. $3^{2}$ (1923) p. $43-67$. [30, $31,33]$.

(ז 0$)$ Theorie und Anwendung der unendlichen Reihen.

Grundlehren d. Math. Wissenschaften, t. 2, Berlin, J. Springer, 1924 (2:e éd.) $\mathrm{X}+527$ p., $8^{\circ}$. [30].

(I I) Über Polynomentwicklungen im Mittag-Lefflerschen Stern durch Anwendung der Eulerschen Reihentransformation.

Acta Math., t. 47 (1926) p. 3I3--335. [25, 3I]. 
Essai d'une bibliographie de la représentation analytique d'une fonction monogène.

\section{H. von Koch.}

(r) Applications nouvelles de la fonction exponentielle.

Bihang t. K. Vet. akad. handl., t. 28 , sect. I, n:o 2 (I902) p. I-r6. [25].

(2) Sur le prolongement analytique d'une série de Taylor.

Acta Math., t. 27 ( 1902$)$ p. $79-$ I04. [25].

(3) Sur une propriété arithmétique du développement en série de Taylor d'une fonetion algébrique.

Arkiv för Math., Astr. o. Fys., t. I (1904) p. 627-64r. [24].

(4) Sur une extension du théorème d'Eisenstein.

Arkiv för Mat., Astr. o. Fys., t. I (I 904) p. 643-650. [24].

(5) Remarques sur quelques séries de polynomes.

Bulletin Soc. Math. de France, t. 34 (I906) p. 269-274. [I I, I 4].

(6) Sur le prolongement d'une série de Taylor.

Arkiv för Mat., Astr. o. Fys., t. r2, n:o I I (1917) 32 p. [25].

(7) Contributions à la théorie du prolongement d'une fonction analytique.

Arkiv för Mat., Astr. o. Fys., t. I 2, n:o 23 (r9ry) 48 p. [25].

(8) Un théorèmè sur les intégrales irrégulières des équations différentielles linéaires et son application au problème de l'intégration.

Arkiv för Mat., Astr. o. Fys., t. 13, n:0 I 5 (1918) I 8 p. [27].

\section{T. Kojima.}

(I) On generalized Toeplitz's theorems on limit and their applications.

Tôhuku Math. Journal, t. I 2 (I9r7) p. 29I-326. [3०, 3I].

\section{J. König.}

(I) Ueber die Darstellung von Functionen durch unendliche Reihen.

Math. Annalen, t. 5 ( 1872$)$ p. 310-340. [13, I 4 ].

(2) Ueber eine Eigenschaften der Potenzreilien.

Math. Annalen, t. 23 (I 884 ) p. $447-449$. [22, 24$]$.

\section{R. König.}

(I) Neuere Funktionentheorie.

Jahresbericht d. Deutschen Math. Verein., t. 33 (I925) p. 74-80. [I०, I4, 25].

\section{Kössler.}

(r) Sur les développements en séries d'une fonction analytique dans un domaine donné, I et II (en tchéque).

Rozpravy české Akad., t. 24, n:o 4I (I925) 34 p. et t. 25, n:o 54 (1926) $3^{8}$ p.

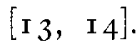

(2) Prịspěvek $\mathrm{k}$ theorii Borelova pokračování funkcí. [= Une remarque sur la théorie de M. Borel pour le prolongement des fonctions.]

Věstnik Král. Čes. Společnosti nauk roč., I $921-22$, N:o VII, I 4 p. [I6, 22, 23$]$. 
(3) Les séries entières avec coupure et leur prolongement au sens de M. Borel (en tchéque).

Rozpravy české Akad., t. $3^{\text {r, n:o }}$ i.9 (r922) 8 p. Resumé française dans le Bulletin Int. de l'Acad. des Sci. de Bohème, t. 24. [16, 23, 28$]$.

(4) Sur les singularités des séries entières situées sur le cercle de convergence:

Bulletin Int. de l'Acad. des Sci. de Bohème, I923, 3 p. [22, 23$]$.

(5) Sur les singularités des séries entières.

Atti R. Accad. Naz. dei Lincei. Rendiconti. 5:e série, t. 32, I:er semestre (1923) p. $26-29$ et $528-53 \mathrm{I}$. $[22,23,24]$.

(6) Nouveaux théorèmes sur les singularités des séries entières.

Atti R. Accad. Naz. dei Lincei. Rendiconti, 5:e série, t. 32, r:er semestre (r923) p. $8_{3}-85 .[23,24]$.

\section{K. I. Koteloff.}

(I) Osobyj vid razloženija holomorfnoj funkcii v bezkonečnoě proizvedenie. [= Sur une classe particulière de résolution d'une fonction holomorphe en produit infinie.] Sobranie. Fiz.-Mat. Naik Obsčestra Est. pri Imp. Kazanskow Univ., t. 8 (1889) p. $407-4$ I 5 . [I3].

\section{Krafft.}

(1) Zur Theorie der Faberschen Polynome und ihrer zugeordneten Funktionen. Thèse, Marburg, I $9 \mathrm{r}_{5}, 69 \mathrm{p} ., 8^{\circ}$. [ [ $\left.3, \mathrm{I}_{3}\right]$.

(2) Potenzreihen auf dem Einheitskreis.

Jahresbericht d. Deutschen Math.-Verein., t. 33 (I925) p. I69-I7I. [22].

\section{Krause.}

(I) Partialbruchzerlegung bei transcendenten Funktionen. Sitzungsberichte Ges. Isis, Dresden, I 899, p. Io-I I. [I3].

\section{Z. Krygowski.}

(r) Sur les fonctions à espaces lacunaires. Bulletin Soc. Math. de France, t. 25 (1897) p. 240-243. [12].

(2) Przyczynek do teoryi funkcyj o obszarach osobliwych. [= Contribution à la théorie des fonctions à espaces lacunaire.] Prace Mat. Fiz., t. 9 (1898) p. 2 I $3-22$ I. [I 2 ].

(3) $\mathrm{Z}$ teoryi funkeyj analitycznych. [= Sur la théorie des fonctions analytiques.] Wiad. Mat., t. 3 (I 899 ) p. I 47 - I54. [I 4$]$.

\section{Kuniyeda.}

(I) Asymptotic formulae for oscillating Dirichlet's integrals and coefficients of powerseries.

Journal Coll. of Sci. Imp. Univ. of Tokyo, t. 4I, art. 4 (I9I9) p. I-I I I. [22, $24,35]$. 
Essai d'une bibliographie de la représentation analytique d'une fonction monogène.

\section{J. Kürschak.}

(I) Az analitikai függvények elméletéhez. [= Sur la théorie des fonctions analytiques.] Math. és Phys. Lapok, t. 4 (1895) p. $24 \mathrm{I}-255$, t. 5 (1896) p. 55-66, I 6 I 28 et I6I-I72. [24].

(2) Über spezielle Funktionenreihen.

Journal für Math., t. I 48 (I9I8) p. I I 3 - I I5. [r4].

\section{E. Laguerre.}

(I) Sur le développement d'une fonction suivant les puissances d'un polynôme. Comptes Rendus, t. 86 ( I I Février I 878 ) p. 383-385. (Euvres t. r, p. 295 297. [I 4 ].

(2) Sur le développement d'une fonction suivant les puissances croissantes d'un polynôme.

Journal für Math., t. 88 (I880) p. 35-48. (Euvres, t. I, p. 298-3I4. [I4].

E. Landau.

(I) Über einen Satz von Tschebyschef.

Math. Annalen, t 6I (1905) p. 527-550. [22].

(2) Über die Grundlagen der Theorie der Fakultätenreihen.

Münchener Berichte, t. 36 (I 906) p. I 5 I-2 2 I 8 . [i 4 ].

(3) Über die Konvergenz einiger Klassen von unendlichen Reihen am Rande des Konvergenzgebietes.

Monatshefte für Math. und Physik, t. I8 (I907).p. 8-28. [22, 33].

(4) Über die Bedeutung einiger neuen Grenzwertsätze der Herren Hardy und Axer. Prace Matematyczno-Fizyczne, t. 2 r (1910) p. 97-177. [33].

(5) Über einen Satz des Herrn Littlewood.

Rendiconti Circ. Mat. di Palermo, t. 35 (I9r3) p. 265-276. [33].

(6) Sur les séries de Lambert.

Comptes Rendus, t. I56 (I 3 Mai r9I3) p. I45 $5^{\mathrm{I}}-\mathrm{r} 454$. [I 2$]$.

(7) Darstellung und Begrïndung einiger neuerer Ergebnisse der Funktionentheorie. Berlin, J. Springer, I9 6 , I I० p., $8^{\circ}$. [20, 22, $23,24,3$ I].

(8) Auszug aus einem Briefe des Herrn Landau an den Herausgeber.

Acta Math., t. 42 (1920) p. 95-98. [25].

\section{P. S. de Laplace.}

(I) Théorie analytique des probabilités.

Euvres, t. $7, \mathrm{CXCV}+69$ I p., $4^{\circ}$.

\section{E. Lasker.}

(I) Über Reihen auf der Convergenzgrenze.

Phil. Transactions R. Soc. of Lonđon, série A, t. I 96 (I90r) p. 431-477. Thèse, 6-2822. Acta mathematica. 52. Imprimé le 24 janvier 1929. 
Erlangen, I900, Résumé dans les Proceedings R. Soc. of London, t. 66 (r900) p. $337-339$. [1 3, 22 ].

\section{S. Lattès.}

(I) Sur les séries de Taylor à coefficients récurrents.

Comptes Rendus, t. I50 (30 Mai I910) p. I4/3-r4I5. [24].

(2) Sur les formes réduites des transformations ponctuelles à deux variables. Applications à une classe remarquable de séries de Taylor.

Comptes Rendus, t. I 52 (6 Juin I 9 I I) p. I 566-i 569. [24].

(3) Sur les formes réduites des transformations ponctuelles dans le domaine d'un point double.

Bulletin Soc. Math. de France, t. 39 (I 9 I I) p. 309-345. [24].

(4) Sur les suites récurrentes non linéaires et sur les fonctions génératrices de ces suites.

Annales de Toulouse, série 3, t. 3 (I 9 I I) p. 73-1 24. [24].

(5) Sur le prolongement analytique de certaines séries de Taylor.

Bulletin Soc. Math. de France, t. 42 (r9I4) p. 95-ir 2. [24, 25$]$.

\section{H. Laurent.}

(r) Sur les séries de polynomes.

Journal de Math., série 5, t. 8 (I902) p. 309-328. [13, I4].

\section{Leau.}

(r) Sur les points singuliers situés sur le cercle de convergence et sur la sommation des séries divergentes.

Comptes Rendus, t. 127 (24 Oct. I 898) p. 607-609. [22, 23, 24, 3 I].

(2) Sur le cercle de convergence des séries.

Comptes Rendus, t. I 27 (7 Nov. 1898) p. 7 r I-7 72 . Avec Erratum à la page 794. $[22,24]$.

(3) Extension d'un théorème de M. Hadamard à l'étude des séries de Taylor.

Bulletin Soc. Math. de France, t. 26 (1898) p. 267-27 r. [24].

(4) Sur les fonctions définies par un développement de Taylor.

Comptes Rendus, t. I 28 (27 Mars 1 899) p. 804-805. [22, 23, 24].

(5) Représentation des fonctions par des séries de polynomes.

Bull. Soc. Math. de France, t. 27 (1899) p. I 94-200. [25].

(6) Recherche des singularités d'une fonction définie par un developpement de Taylor. Journal de Math. pures et appl. série 5, t. 5 (1899) p. 365-425. [22, 23,24$]$.

\section{Lecornu.}

(r) Sur les séries entières.

Comptes Rendus, t. ro4 (7 Février r 887) p. 349-352. [2 r, 22 ]. 
Essai d'une bibliographie de la représentation analytique d'une fonction monogène. 43

\section{G. Lejeune-Dirichlet.}

( par M. Liouville.

Journal de Math., 2:e série, t. 7 (1862) p. 253-255. [22].

\section{Lerch.}

(1) Note sur les expression qui, dans diverses parties du plan, représentent des fonctions distinctes.

Bulletin Sci. Math., série 2, t. Io (1886) p. 45-49. [I I].

(2) Contributions à la théorie des fonctions.

Sitzungsberichte K. Böhmischen Ges. d. Wissenschaften, Prag, I886, p. 57 I582. [23].

(3) Un théorème de la théorie des séries. Acta Math., t. Io (r887) p. $87-88$. [23].

(4) Ueber Funktionen mit beschränktem Existenzbereiche.

Abhandlungen K. Böhmischen Ges. d. Wis., 7:e série, t. 2, n:o 9 ( 1888 ) 20 p. $\left[\begin{array}{ll}12 & 23\end{array}\right]$.

(5) Ueber die Nichtdifferentiirbarkeit gewisser Functionen.

Journal für Math., t. Io3 (1888) p. I26-1 38 . [23].

(6) Sur une classe de fonctions à espace lacunaire.

Jornal de Sciencias Math. e Astr., t. Io (189o) p. 27-28. [1 2].

(7) Ueber die analytische Natur einer von P. du Bois-Reymond betrachteten Function.

Monatshefte für Math. u. Phys., t. 8 (1897) p. 377-382. [24].

(8) Sur la nature de la fonction $\sum_{m=1}^{\infty} u^{m} e^{-2} \sqrt{a m}$ dans le voisinage du point $u=\mathbf{I}$ (en tchéque).

Časopis, t. 39 (I 9 Io) p. I 2 I-I 33. [24].

(9) Remarques comparatives sur les séries de M. Fredholm et de M. Du Bois-Reymond. Une contribution à la détermination du domaine d'existence des fonctions analytiques d'une nature speciale (en tchéque).

Casopis, t. 39 (1910) p. $225-236$. [1 2, 23].

\section{É. Le Roy.}

(I) Sur les points singuliers d'une fonction définie par un développement de Taylor. Comptes Rendus, t. I 27 (5 Déc. r 898) p. 948-95o. [24].

(2) Sur les séries divergentes et les fonctions définies par un développement de. Taylor.

Comptes Rendus, t. I 27 ( 3 r Oct. 1898) p. 654-657, et t. i 28 (20 Février I 899) p. $492-495$. $[24,25,34]$. 
(3) Sur les séries divergentes.

Comptes Rendus, t. I 30 (I 4 Mại rgoo) p. I 293 - I 296 et (5 Juin I900) p. I 535 -I 536 . $[22,3 \mathrm{r}]$.

(4) Sur les séries divergentes et les fonctions définies par un développement de Taylor. Annales de Toulouse, série 2, t. 2 (1900) p. 3I 7 -430. $[24,25,3 \mathrm{I}]$.

(5) Valeurs asymptotiques de certaines séries procédant suivant les puissances entières et positives d'une variable réelle.

Bulletin Sci. Math., 2:e série, t. 24 (1900) p. 245-268. [22].

\section{P. Lévy.}

(I) Remarques sur les procédés de sommation des séries divergentes.

Comptes Rendus, t. I 82 (29 Mars i 926 ) p. $835-838$. [30].

(2) Sur les conditions d'application et sur la régularité des procédés de sommation des séries divergentes.

Bulletin Soc. Math. de France, t. 54 (1926) p. I-25. [30].

\section{W. Lewicki.}

(ז) Zur Theorie der Potenzreihen.

Monatshefte für Math. und Physik, t. i 2 (r9or) p. 329-336. [23].

(2) Sur la théorie des séries entières (en ukrainien).

Naukove tovarystvo imeni Ševčenka. Lwów. Zbirnyk mat.-pryd.-likars'koi sekcii, t. 7, n:o I $(\mathrm{r} 900)$ Io p. [2 I ?].

(3) Les dernières conquêtes dans la théorie des fonctions analytiques (en ukrainien). Ibid., t. 7, n:0 4 (1901) I 2 p. [24, 25$]$.

J. W. Lindeberg.

(I) Sur l'existence de fonctions d'une variable complexe et de fonctions harmoniques bornées.

Annales Acad. Sci. Fennicae (A), t. I I, n:o 6 (I918) 27 p. [I3].

\section{E. Lindelöf.}

(r) Sur la transformation d'Euler et la détermination des points singuliers d'une fonction définie par son développement de Taylor.

Comptes Rendus, t. I 26 (28 Février r898) p. 632-634. [24, 3 I].

(2) Remarques sur un principe général de la théorie des fonctions analytiques. Acta Soc. Sci. Fennicae, t. 24, n:0 7 (1898) p. I-39. $[24,25,3 \mathrm{r}]$.

(3) Sur le prolongement analytique.

Bull. Soc. Math. de France, t. 29 (I90I) p. I $57-$ I60. [25].

(4) Une application de la théorie des résidus au prolongement analytique des séries de Taylor.

Comptes Rendus, t. I35 (29 Déc. I902) p. I3 I5-i318. [25]. 
(5) Quelques applications d'une formule sommatoire générale. Acta Soc. Sci. Fennicae, t. 3 I, n:0 3 (I902) p. I-46. [25, 3 I].

(6) Sur la détermination de la croissance des fonctions entières définies par un développement de Taylor.

Bull. Sci. Math., 2:e série, t. 27 (1903) p. 2 I $3^{-226}$. [32].

(7) Sur l'application de la théorie des résidus au prolongement analytique des séries de Taylor.

Journal de Math., série 5, t. 9 (1903) p. 2 I3-2 2 I. [25].

(8) Sur une formule sommatoire générale. Acta Math., t. 27 (1903) p. 305-3I I. [25, 3I].

(9) Le calcul des résidus et ses applications à la théorie des fonctions. Paris, Gauthier-Villars, I 905 , VI + I 43 p., $8^{\circ}$. [I०, 24, 25,32$]$.

\section{J. E. Littlewood.}

(I) The converse of Abel's theorem on power series. Proceedings Londion Math. Soc., série 2, t. 9 (I9II) p. 434-448. [22, 33].

\section{E. Lugaro.}

(r) Intorno alle singolarità di una funzione dipendenti da quelle di più funzioni date. Periodico di Mat., 3:e série, t. I (I904) p. Iо5- I 23. [24].

\section{F. Lucács.}

(I) Bemerkung zu einem Konvergenzsatze des Herrn Landau. Archiv d. Math. u. Phys., 3:e série, t. 23 (1915) p. $367-368$. [33].

\section{J. Lüroth}

(1) Eine historische Bemerkung zur Funktionentheorie. Math. Annalen, t. 60 (I905) p. 398-40I. [II].

\section{N. Lusin.}

(I) Über eine Potenzreihe. Rendiconti Circ. Mat. di Palermo, t. 32 (r9ri) p. 386-39o. [22].

(2) Ob odnom slučaě rjada Taylor'a. [= Sur un cas particulier de la série de Taylor.] Mat. Sbornik ezd. Moskovskim Mat. Obščestvom, t. 28 (IgII-I2) p. 295-302. $[22]$.

\section{N. Lusin et J. Priwaloff.}

(I) Sur l'unicité et la multiplicité des fonctions analytiques.

Comptes Rendus, t. I 78 (28 Janvier I924) p. 456-459. [22]. 


\section{E. Maillet.}

(r) Sur les séries divergentes et les équations différentielles. Comptes Rendus, t. I34 (28 Avril 1902) p. 975-977. [27, 3I].

(2) Sur les séries divergentes et les équations différentielles.

Annales de l'École Normale, 3:e série, t. 20 (1903) p. 487-5r8. $[25,27,31]$.

\section{J. Malmquist.}

(1) Sur le calcul des intégrales d'un système d'équations différentielles par la méthode de Cauchy-Lipschitz.

Arkiv för Mat., Astr. o. Fysik, t. I, N:o Io (I903) p. I49-I56. [27].

(2) Étude d'une fonction entière.

Acta Math., t. 29 (1905) p. 203-2 I 5. [32].

\section{S. Mandelbrojt.}

(I) Sur les séries de Taylor qui ont des lacunes.

Comptes Rendus, t. I 76 (I 2 Mars I923) p. 728-730; (9. Avril I923) p. $978-$ 98 I. [24].

(2) Quelques théorèmes sur les séries entières.

Comptes Rendus, t. 177 (1 0 Déc. I 923 ) p. I 27 I-I $273 .[23,24]$.

(3) Sur les séries de Taylor qui présentent des lacunes.

Annales de l'École Norm., série 3, t. 40 (1923) p. 4I3-462. Aussi Thèse, Paris, Gauthier-Villars, I 923,50 p., $4^{\circ} .[23,24]$.

(4) Sur les séries d'Eisenstein.

Comptes Rendus, t. I 78 (I 7 Mars I924) p. $985-987$. [24].

(5) Sur les séries de Taylor prolongeables.

Comptes Rendus, t. I 78 (2 Juin I 924 ) p. I $873-1875$. [24].

(6) Quelques théorèmes sur le nombre des points singuliers d'une série entière. Comptes Rendus, t. I8 I (6 Juillet I925) p. $20-22$. [24].

(7) Sur la meilleure approximation des fonctions analytiques et leurs points singuliers.

Comptes Rendus, t. I 8 I (2 I Sept. I 925 ) p. 366-368. [ [ 5, 24 ].

(8) Sur la définition des fonctions analytiques. Acta Math., t. 45 (1925) p. I $29-143$. [24].

(9) Quelques généralisations des théorèmes sur les séries qui admettent des lacunes. Comptes Rendus, t. I82 (4'Janvier 1926) p. 38-39. Erratum p. 548. [24].

(10) Les nombres transcendants et les fonctions analytiques.

Comptes Rendus, t. I 82 (1:er Février 1926) p. 305-307. Errata p. 548. [24].

(x) Sur la détermination effective des points singuliers d'une fonction analytique donnée par son développement en série de puissances.

Comptes Rendus, t. I82 (I 5 Février I926) p. $437-439$. [24]. 
Essai d'une bibliographie de la représentation analytique d'une fonction monogène.

(12) La recherche des points singuliers d'une fonction analytique représentée par une série des puissances.

Journal de Math., 9:e série, t. $5(1926)$ p. I97--2 10. [24].

(13) Sur les séries de Taylor qui ont des lacunes généralisées.

Bulletin Soc. Math. de France, t. 53 (1926) p. 235-245. [24].

\section{P. Martinotti.}

(I) Su le serie d'interpolazione.

R. Ist. Lombardo. Rendiconti, 2 :e série, t. 43 (r9Io) p. $39 \mathrm{I}-40 \mathrm{I}$. [I4].

(2) Ulteriori ricerche su le serie d'interpolazione.

R. Ist. Lombardo. Rendiconti, $2: e$ série, t. 43 (1910) p. 556-569. [I 4 ].

(3) Su la convergenza dei polinomi e delle serie d'interpolazione.

R. Ist. Lombardo. Rendiconti, 2 :e série, t. 43 (I9Io) p. 760-770. [I4].

\section{T. Matsumoto.}

(I) Sur la série entière dont quelques sommes partielles convergent en dehors du cercle de convergence.

Tôhuku Math. Journal, t. $27(1926)$ p. 243-247. [23, 24].

\section{S. Mazurkiewicz.}

(r) O niesumowalnych szeregach potęgowych i trygonometrycznych. [ = Sur les séries entières et les séries trigonométriques non-sommables.]

Prace matematyczno-fizyczne, t. 28 (I9I7) p. I09-II8. [22].

(2) Sur les séries de puissances.

Fundamenta Math., t. 3 (1922) p. 52-58. [22].

W. Meierhöfer.

(x) Über die Konvergenz von Potenzreihen auf dem Konvergenzkreise.

Thèse, München, I 920,24 p. [22].

\section{Hj. Mellin.}

(I) Die Theorie der asymptotischen Reihen vom Standpunkte der Theorie der reziproken Funktionen und Integrale.

Annales Acad. Sci. Fennicae A, t. I8, n:o 4 (1922) ro8 p. [34].

(2) Abriss einer allgemeinen und einheitlichen Theorie der asymptotischen Reihen. Wissenschaftliche Vorträge geh. auf d. Fünften Kongress der Skand. Math. in Helsingfors I922. Helsingfors, I923, p. I- I 7. [34].

(3) Anwendung einer allgemeinen Methode zur Herleitung asymptotischen Formeln. Annales Acad. Sci. Fennicae, A, t. 20, n:o I (I924) 44 p. [22, 34].

(4) Ueber die analytische Fortsetzung von Funktionen, welche durch gewisse allgemeine Dirichletsche Reihen definiert sind.

Annales Acad. Sci. Fennicae, A, t. 20, n:o Io (1924) 60 p. $[24,25]$. 


\section{Ch. Méray.}

(r) Observations sur la légitimité de l'interpolation.

Annales de l'École Normale, 3:€ série, t. I (1884) p. 165-I76. [14].

(2) Sur l'impossibilité de franchir par la formule de Taylor les cercles de convergence de certaines séries entières.

Bulletin Sci. Math., 2 :e série, t. I 2 (1 888 ) p. $248-252$. [23].

\section{Mignosi. ${ }^{1}$}

(I) Inversione d'un teorema sul rapporto delle medie $(C p)$ di due serie.

Rendiconto Accad. Sci. fis. e mat. di Napoli, 3 :e série, t. 27 (192 I) p. I $7-28$. [33].

(2) Sulle medie di Doetsch delle funzioni.

Note e memorie di mat., Catania, t. I (I92I) p. I36-is8. [3 I].

\section{S. Minetti.}

(1) Sul raggio di convergenza degli sviluppi tayloriani $\Sigma a_{n} z^{n}$ ove $a_{n}=g(n)$ per $n$ intero positivo con $g(n)$ trascendente intera.

Atti R. Accad. Naz. dei Lincei. Rendiconti, 6:e série, t. 3 (I926) p. $723^{-} 73$ I. $[2 \mathrm{I}, 24]$.

(2) Sulla ricerca della singolarità delle $f(z)=\Sigma a_{n} z^{n}$ ove $a_{n}=g(n)$ per $n$ intero positivo con $g(n)$ trascendente intera.

Atti R. Accad. Naz. dei Lincei. Rendiconti, 6:e série, t. 4 (I926) p. I $4^{-1} 7$. [24].

(3) Ancora sugli sviluppi tayloriani di $\Sigma a_{n} z^{n}$ dove $a_{n}=g(n)$ con $g(n)$ trascendente intera.

Atti R. Accad. Naz, dei Lincei. Rendiconti, 6:e série, t. 4 (I926) p. r I 3. [24].

(4) Sur le rayon de convergence et sur les singularités d'une classe de fonctions analytiques définies par le développement de Taylor.

Comptes Rendus, t. I82 (28 Juin 1926) p. I 595-I 597. [2 I, 24].

(5) Sur le rayon de convergence et sur les singularités de certains développements tayloriens et des fonctions analytiques qu'ils définissent.

Comptes Rendus, t. I83 (I7 Aôti I926) p. 4I4-4I5. [21, 24 ].

\section{G. Mittag-Leffler.}

(1) En metod att analytiskt framställa en funktion af rationel karakter, hvilken blir oändlig alltid och endast uti vissa föreskrifna oändlighetspunkter, hvilkas konstanter äro på förhand angifna. [= Méthode pour la représentation analy. tique d'une fonction de caractère rationnel qui devient infinie seulement en des points donnés dont les constantes sont données.]

Öfversigt K. Vetenskaps-Akad. Förhandl., t. 33, n:o 6 (I876) p. 3- - 6. [I3, r4].

(2) Ytterligare om den analytiska framställningen af funktioner utaf rationel karakter. [ = Remarques additionelles sur la représentation analytique des fonctions de caractère rationnel.]

Öfversigt K. Vetenskaps-Akad. Förhandl,, t. 34, n:o I (1877) p. r7 -32 . [I3, I4].

1 Voir p. 80. 
Essai d'une bibliographie de la représentation analytique d'une fonction monogène.

(3) Om den analytiska framställningen af en funktion af rationel karakter med en godtyckligt vald gränspunkt. [ = Sur la représentation analytique d'une fonction de caractère rationnel ayant un point limite arbitrairement choisi.]

Öfversigt K. Vetenskaps-Akad. Förhandl., t. 34, n:o I (I877) p. 33-43. [I3, I 4].

(4) Om den analytiska framställningen af en funktion af rationel karakter med ett ändligt antal godtyckligt förskrifna gränspunkter. [= Sur la représentation analytique d'une fonction de caractère rationel ayant un nombre fini de points limites arbitrairement choisis.]

Öfversigt K. Vetenskapsakad. Förhandl., t. 34, n:o 2 (I877) p. 3I-4I. [I 3, I 4].

(5) Till frågan om den analytiska framställningen af en funktion af rationel karakter genom qvoten af två beständigt konvergerande potensserier. [= Sur la représentation analytique d'une fonction de caractère rationnel par le quotient de deux séries entières partout convergentes.]

Öfversigt K. Vetenskaps-Akad. Förhandl., t. 34, n:o 3 (I877) p. 5-г3. [I3].

(6) Om den analytiska framställningen af funktioner af rationel karakter utaf flere oberoende variabler. I, II. [ = Sur la représentation analytique des fonctions de caractère rationnel de plusieurs variables indépendantes.]

Öfversigt K. Vetenskaps-Akad. Förhandl., t. 34, n:o ro (I877) p. 3-I 5, I 3 r. [I3].

(7) Extrait d'une lettre à M. Hermite.

Bulletin Sci. Math., série 2, t. 3 (1879) p. 269-278. [I3, I4].

(8) Funktionsteoretiska studier. I. En ny serieutveckling för funktioner af rationel karakter. [= Recherches sur la théorie des fonctions. I. Un nouveau développement en série pour les fonctions de caractère rationnel.]

Acta Soc. Sci. Fennicae, t. I I (I879) p. 275-293. [I3, I4].

(9) Några funktionsteoretiska undersökningar. [= Quelques recherches sur la théorie des fonctions.]

Öfversigt Finska Vet.-Soc:s Förhandl., t. 23 (I88 I) p. 95-99. [II].

(ro) Recherches sur la théorie des fonctions.

Bulletin Sci. Math., z:e série, t. 5 (I $88 \mathrm{I}$ ) p. 388-392. [I I].

(I I) Fullständig analytisk framställning af hvarje entydig monogen funktion, hvars singulära ställen utgöra en värdemängd af första slaget. [= Représentation analytique complète de toute fonction monogène uniforme dont les points singuliers forment un ensemble de la première classe.]

Öfversigt K. Vetenskaps-Akad. Förhandl., t. 39, n:o 2 (I882) p. I I-45. [I 3, r 4].

(r 2) Om den analytiska framställningen af en entydig monogen funktion hvilken uti omgifningen af hvarje punkt, som är belägen innanföre en viss cirkelperiferi, endast har ett ändligt antal singulära ställen. [=- Sur la représentation analytique d'une fonction monogène uniforme qui n'admet qu'un nombre fini de points singuliers dans le voisinage de chaque point intérieur à un certain cercle.]

Öfversigt K. Vetenskaps-Akad. Förhandl., t. 39, n:0 4 (I882) p. 2 I-24. [I 3, I4].

7 - 2822. Acta mathematica. 52. Imprimé le 25 janvier 1929. 
(r3) Sur la théorie des fonctions uniformes d'une variable. Comptes Rendus, t. 94 (I3 Février I 882) p. 4I4-4I6; (20 Février) p. 5I I514; (I3 Mars) p. 7 I3-7 5 ; (20 Mars) p. 78I-783; (3 Avril) p. 938-94I; (1о Avril) p. I040-1042; (I 7 Avril) p. I 105-I 107; (24 Avril) p. I 63 - II65; et t. 95 (I4 Août I882) p. 335-336. [I3, I4].

(14) Sur la représentation analytique des fonctions monogènes uniformes d'une variable indépendante.

Acta Math., t. 4 ( 1884 ) p. I- 79 . [I 3, I 4 ].

(15) Sur une transcendante remarquable découverte par M. Fredholm.

Comptes Rendus, t. i io (24 Mars i 890) p. 627-629. [23].

(ı) Sur une transcendante remarquable trouvée par M. Fredholm. Lettre de M. Mittag-Leffler à M. Poincaré.

Acta Math., t. I5 (189i) p. 279-280. [23].

( 17 ) $\mathrm{Om}$ en generalisering af potensserien. $[=$ Sur une généralisation de la série entière.]

Öfversigt K. Vet.-Akad. Förhandl., t. 55 (r 898$)$ p. I $35^{--}$r.38. [25].

(г8) Om den analytiska framställningen af en allmän monogen funktion. [ $=-$ Sur la représentation analytique d'une fonction monogène générale.]

Öfversigt K. Vet.-Akad. Förhandl., t. 55 (x 898$)$ p. $247^{--262,263-282}$ et 375 $-385 \cdot[25]$.

(19) Sur la représentation d'une branche uniforme de fonction analytique. Comptes Rendus, t. I 28 ( I 5 Mai I 899) p. I 2 I 2 -I 2 I 5. [25].

(20) Sulla rappresentazione analitica di un ramo uniforme di una funzione monogena. Atti R. Accad. di Torino, t. 34 (1899) p. 48I-49 I. [25].

(2 I) Sur la représentation analytique d'une branche uniforme d'une fonction monogène. (Première note.)

Acta Math., t. 23 (1899) j. 43-62. [25].

(22) Sur la représentation analytique d'une branche uniforme d'une fonction monogène. (Seconde note.)

Acta Math., t. 24 (1900) p. I $83-204$. [25].

(23) Sur la représentation analytique d'une branche uniforme d'une fonction mono. gène. (Troisième note.)

Acta Math., t. 24 (1900) p. $205-244$. [25, 26].

(24) On the analytical representation of a uniform branch of a monogenic function. Transactions Cambridge Phil. Soc., t. I 8 (I900) p. I-II. [25].

(25) On multiply infinite series and on an extension of Taylor's series. Proceedings London Math. Soc., t. 32 (1900) p. 72-78. [25].

(26) Ueber eine Verallgemeinerung der Taylorschen Reihe. Göttinger Nachrichten I900 p. I94-205. [25].

(27) Sur une formule de M. Fredholm.

Comptes Rendus, t. I 32 (25 Mars r9oI) p. $75 \mathrm{I}-753$. [25]. 
Essai d'une bibliographie de la représentation analytique d'une fonction monogène.

(28) Sur la série de Bernoulli.

Comptes Rendus, t. I 32 ( Io Juin I 90I) p. I $388-$ - 39 I. [25].

(29) Über den Konvergenzbereich der Bernoullischen Reihe. (Voir C. R. Dietrich.) Archiv der Math. u. Physik, série 3, t. 2 (I90I) p. 49-54. [25].

(30) Un critère pour reconnaître les points singuliers de la branche uniforme d'une fonction monogène.

Comptes Rendus, t. I33 (I 2 Aô̂t r 90 r) p. 357-36r. [24].

(3I) A criterion for the recognition of the irregular points of analytic functions.

Report British Ass. for the Advancement of Science, I 901, p. 549-550. [24].

(32) Analytische Darstellung monogener Funktionen von mehreren unabhängigen Veränderlichen.

Jahresbericht d. Deutschen Math. Verein., t. 9 (I90I) p. 74-77. [25].

(33) Sur le terme complémentaire de mon développement de la branche uniforme d'une fonction monogène dans le cas où ce développement possède une étoile de convergence.

Öfversigt K. Vetenskapsakad. Förhandl., t. 58 (I90 I) p. $785-790$. [25].

(34) Sur une extension de la série de Taylor.

Compte Rendu du deuxième Congrès International des mathématiciens, p. 273 -276 . Paris, Gauthier-Villars, I902. [25].

(35) Sur la représentation analytique d'une branche uniforme d'une fonction monogène. (Quatrième note.)

Acta Math., t. 26 (1902) p. 353-391. [25].

(36) Sur l'intégrale de Laplace-Abel.

Comptes Rendus, t. I 35 ( I:er Déc. r902) p. 937-939. [25, 3I, 32].

(37) Une généralisation de l'intégrale de Laplace-Abel.

Comptes Rendus, t. 136 (2 Mars I903) p. 537-539. [25,31, 32].

(38) Sur la nouvelle fonction $E_{\alpha}(x)$.

Comptes Rendus, t. I 37 (I 2 Oct. I903) p. 554-558. [32].

(39) Sopra la funzione $E_{c x}(x)$.

Atti R. Ac. Lincei. Rendiconti, série 5, t. I 3 (I904) p. 3-5. [32].

(40) Un nouveau théorème général de la théorie des fonctions analytiques. Comptes Rendus, t. I 38 (I I Avril I904) p. 88 I -884 . [25].

(4I) Une nouvelle fonction entière. Comptes Rendus, t. I38 (r8 Avril I904) p. 94I-942. [32].

(42) Sur une classe de fonctions entières. Verhandlungen d. 3. intern. Math. Kongr. Heidelberg igo4 p. $258-264$. [32].

(43) Sur la représentation analytique d'une branche uniforme d'une fonction monogène (cinquième note).

Acta Math., t. $29(1905)$ p. Ior- I 8 I. $[25,32]$.

(44) O przedstawieniu analitycznem jednoznadznej gałęzi funkcyi analitycznej. [= Sur la représentation analytique d'une branche uniforme d'une fonction monogène. Traduction polonaise par M. S. Dickstein.] 
Prace mat.fiz., t. I6 (1905) p. I $57-$ I 75, I 75 - I96, I $97-232 ;$ t. I 7 (1906) p. $223^{-2} 58$, et t. 18 (I907) p. $8 \mathbf{I}-\mathbf{I} 4 \mathrm{I} \cdot[25,26]$.

(45) Sur la représentation arithmétique des fonctions analytiques générale d'une variable complexe.

Atti del IV Congrezzo int. dei Mat. Roma i 908, t. I p. 67-85. $[25,26,32]$.

(46) Sur un problème d'Abel. (Extrait d'une lettre à M. Marcel Riesz.)

Rendiconti Circ. Mat. di Palermo, t. 30 (I9I0) p. 337-338. [26].

(47) Grundläggande satser inom teorien för integralen $I(t)=\int_{0}^{\infty} e^{-\Theta(v) t} F(\nu) d \nu$. [ = Théorèmes fondamentaux dans la théorie de l'intégrale . . . . . ]

Beretning om den 3. skand. mat. kongress. Kristiania 1913. Oslo, ig r 5, p. 37 -60 .

(48) Über die analytische Darstellung eines eindeutigen Zweiges einer monogenen Funktion. (Avec une addition: Ein Satz des Herrn Marcel Riesz, p. I6I-I64. [26].) Münchener Berichte, I9I5, p. I09-164. [25, 26].

(49) Über einen Satz des Herrn Serge Bernstein. Münchener Berichte, 19 I 5 , p. 4I9-424. [1 5,25$]$.

(50) Sur un théorème de M. Serge Bernstein. Tôhuku Math. Journal, t. 9 (I9I6) p. I-6. [ [ 5,25$]$.

(5 I) Sur la représentation analytique d'une branche uniforme d'une fonction monogène. (Sixième note.) Acta Math., t. 42 (19I9) p. $285^{--3} 308$. [25].

(52) Om lineär fortsättning af analytiska funktioner. [ = Sur le prolongement linéaire des fonctions analytiques.]

Matematisk Tidskrift B, I9 I 9, p. 29-33. [14, 28].

(53) Sur la série de Dirichlet et la série de facultés. Acta Math., t. 46 (1925) p. 337-340. [1 4, 25].

\section{J. Mollerup.}

(I) Une méthode de sommabilité par des moyennes éloignées.

Danske Videnskab. Selskab. Meddelelser, t. 3, n:o 8 (1920) 29 p. [22, 3 I, 33].

\section{P. Montel.}

(I) Sur les suites infinies de fonctions.

Thèse, Paris, Gauthier-Villars I907, 103 p. 4, et Annales de l'École Norm., série 3, t. 24 (1907) p. 233-334. [14].

(2) Leçons sur les séries de polynomes à une variable complexe.

Paris, Gauthier-Villars, I9I0, VI + I 28 p. $8^{\circ}$. [ [ 3, I 4, 25$]$.

(3) Sur les séries de Taylor présentant des lacunes.

Comptes Rendus des séances Soc. Math. de France. (27 Mai ig25) p. 33. [23]. 
Essai d'une bibliographie de la représentation analytique d'une fonction monogène.

\section{R. Montessus de Ballore.}

(I) Sur la représentation des fonctions par des suites de fractions rationnelles. Comptes Rendus, t. I 38 (22 Février 1904) p. 47 I-474. [I3, I 4].

\section{N. Moore.}

(I) On the introduction of convergence factors into summable series and summable integrals.

Transactions American Math. Soc., t. 8 (1907) p. 299-330. [22, 3I].

(2) Sur la relation entre certaines méthodes pour la sommation d'une série divergente. Comptes Rendus, t. I58 (I5 Juin r9I4) p. r774-I775. [3I].

(3) Applications of the theory of summability to developments in orthogonal functions. Bull. Amer. Math. Soc., t. 25 (19x8-r9) p. $258-276 .[22,3 \mathrm{I}]$.

(4) Generalized limits in general analysis. I, II.

Trans. Amer. Math. Soc., t. 24 (I922) p. $79^{-88}$ et t. 25 (1923) p. $459-468 \cdot[30,3$ I].

G. Morera.

(I) Sulla rappresentazione delle funzioni di una variabile complessa per mezzo di espressioni analitiche infinite.

Atti R. Accad. Sci. di Torino, t. 2 I (I886) p. 892-899. [I I, I3].

(2) Un teorema fondamentale nella teorica delle funzioni di una variabile complessa. Rendiconti R. Ist. Lombardo, 2:e série, t. I9 (I886) p: 304-307. [13].

(r) Sulle serie di Dirichlet.

\section{Pia Nalli.}

Rendiconti Circ. Mat. di Palermo, t. 40 (I9I5) p. 44-70, I67-I68. [31].

(2) Sulla sommabilità delle serie, con particolare riguardo alle serie di Dirichlet. Rendiconti Circ. Mat. di Palermo, t. 42 (IgI6) p. 6I-72. [3I].

(3) Sopra una relazione fra la teoria della composizione di prima specie e lo studio delle serie divergenti.

Rendiconti Circ. Mat. di Palermo, t. 42 (I9I7) p. 206-226. [3I].

\section{Neder.}

(I) Zur Konvergenz der trigonometrischen Reihen, einschliesslich der Potenzreihen auf dem Konvergenzkreise.

Thèse. Göttingen, I9I 9, $45 \mathrm{p}, 8^{\circ}$. [22].

(2) Konvergenzdefekte der Potenzreihen stetiger Funktionen auf dem Rande des Kon vergenzkreises.

Math. Zeitschrift, t. 6 (I920) p. $262-269$. [22].

(3) Zur Theorie der trigonometrischen Reihen.

Math. Annalen, t. 84 (I921) p. I I $7-$ I 36 . [2 2].

(4) Über Taubersche Bedingungen.

Proceedings London Math. Soc., 2:e série, t. 23 (I924) p. I72-184. [22,31,33]. 


\section{F. Nevanlinna.}

(1) Zur Theorie der asymptotischen Potenzreihen.

Ann. Acad. Sci. Fennicae (A), t. i 2, n:0 3 (19 r6) 8 I p. [34].

(2) Über die Summation der Fourier'schen Reihen und Integrale.

Översikt Finska Vet.-Soc. Förhandl., t. 64, Sect. A, n:o 3 (r920-2 r) I 4 p. [3I].

(3) Zur Theorie der asymptotischen Potenzreihen. Zweite ergänzende Abhandlung. Annales Acad. Sci. Fennicae, A, t. r6, n:o 8 (I922) 26 p. [34].

\section{$F$. et $R$. Nevanlinna.}

(I) Über die Eigenschaften analytischer Funktionen in der Umgebung einer singulären Stelle oder Linie.

Acta Soc. Sci. Fennicae, t. 50, n:o $5(1922) 46$ p. [1 2, 22$]$.

\section{R. Nevanlinna.}

(I) Kriterien für die Randwerte beschränkter Funktionen.

Math. Zeitschrift, t. I 3 ( I922) p. I- - 9. [22].

(2) Asymptotische Entwicklungen beschränkter Funktionen und das Stieltjesche Momentenproblem.

Annales Acad. Sci. Fennicae (A), t. I 8, n:o 5 (1922) 53 p. [34].

\section{N. Nielsen.}

(I) Sur quelques transformations d'une série de puissances. Annali di Mat., 3 :e série, t. Io ( 1904$)$ p. I $47-\mathrm{I}_{5} 6 .[2 \mathrm{I}]$.

\section{N. E. Nörlund.}

(1) Sur les séries de facultés. Comptes Rendus, t. I 58 (4 Mai I9 I4) p. I $25^{2-x} 253$. [I4, 3I].

(2) Sur les séries de facultés et les méthodes de sommation de Cesàro et de $\mathrm{M}$. Borel Comptes Rendus, t. I $5^{8}$ (I I Mai I9I4) p. I $3^{2} 5^{- \text {I } 327 . ~[I 4, ~ 3 I, ~ 34] . ~}$

(3) Sur les séries de facultés. Acta Math., t. 37 (i 914) p. 327-387. [I 4; 3 I, 34].

(4) Sur une application des fonctions permutables. Lunds Universitets Årsskrift. Ny följd. Avd. 2, t. I6, n:o 3 (1920) 1o p. [3 I].

(5) Mémoire sur le calcul aux différences finies. Acta Math., t. 44 (I922) p. 7 I-2 I 2. [3 I, 34].

(6) Sur les formules d'interpolation de Stirling et de Newton. Annales de l'École Norm., 3:e série, t. 39 (1922) p. $343-403$; t. 40 (1923) p. $35-54$. [I 4].

(7) Vorlesungen über Differenzenrechnung.

Die Grundlehren der Math. Wiss., t. I3, Berlin, J. Springer, r924, IX +55 I p., $8^{\circ}$. [I $\left.4,3 \mathrm{I}, 34\right]$. 


\section{N. Obreschkoff.}

(I) Über einige Konvergenzsätze. Jahresbericht d. Deutschen Math. Verein., t. 33 (1925) p. 125-127. [22].

(2) Sur la sommation des séries divergentes et le prolongement analytique. Comptes Rendus, t. I 82 (1:er Février 1926) p. 307-309. [25, 31].

\section{Y. Okada.}

(I) Über die Annäherung analytischer Funktionen. Japanese Journal of Math., t. I (1924) p. 37-40. [25, 3 I ].

(2) Über die Annäherung analytischer Funktionen. Math. Zeitschrift, t. $23(1925)$ p. $62-71$. [25, 3i $]$.

\section{H. Oldenburg.}

(s) En anmärkning angående s. k. divergenta summerbara serier. [= Remarque sur les séries dites divergentes et sommạbles.]

Öfversigt K. Vetenskapsakad. Förhandl., t. 55 (I898) p. I57-I62. [3I].

\section{W. Oseen.}

(I) Sur un théorème de M. Le Roy. Arkiv för Mat., Astr. o. Fys, t. 2, n:0 2 I (1906) p. 1-6. [24, 25$]$.

(2) Eine Bemerkung über die analytische Fortsetzung von Funktionen mehrerer Veränderlichen.

Arkiv för Mat., Astr. o. Fys., t. 3, n:o 2 I (1907) p. r-3. [1 I, 25].

(3) Sur une application des méthodes sommatoires de MM. Borel et Mittag-Leffler.

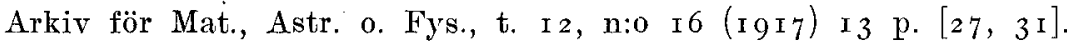

(4) Zwei Bemerkungen über das Problem, eine Taylorsche Reihe analytisch fortzusetzen.

Arkiv för Mat., Astr. o. Fys., t. r3, n:o ro (r9i8) 24 p. [25].

(5) Verallgemeinerung eines funktionentheoretischen Satzes von Herrn H. v. Koch. Arkiv för Mat., Astr. o. Fys., t. I3, n:o I I (I9 18) 7 p. [25].

(6) Sur la représentation analytique de la vitesse dans certain problèmes d'hydrodynamique.

Nova Acta Reg. Soc. Sci. Upsaliensis, sér. 4, t. 4, fasc. 2, n:o 9 (I9I7) p. I$77 \cdot[25,27]$.

\section{W. F. Osgood.}

(1) Example of a single-valued function with a natural boundary, whose inverse is also single-valued.

Bulletin American Math. Soc., t. 4 (1898) p. 4I 7-424. Note supplémentaire, t. 5 (г898) p. $\quad 7^{-1} 8$. [23].

(2) Note on the generalization of Poincaré and Goursat's proof of a theorem of Weierstrass's.

Bulletin American Math. Soc., t. 5 (1898) p. I4-I7. [13]. 
(3) Selected topics in the general theory of functions. Bulletin American Math. Soc., t. 5 (1898) p. 59-87. [13, 24].

(4) Allgemeine Theorie der analytischen Funktionen a) einer und b) mehrerer komplexen Grössen.

Encyklopädie d. math. Wissenschaften, t. 2:2 (1902) p. I-I I4. [Io].

(5) Lehrbuch der Funktionentheorie. I.

Leipzig, B. G. Teubner, I9 г 2 (z:e éd.) XII +766 p., $8^{\circ}$. [го, 20].

\section{A. Ostrowski.}

(I) Über eine Eigenschaft gewisser Potenzreihen mit unendlich vielen verschwindenden Koeffizienten.

Berliner Sitzungsberichte (1921) p. $557-565$. [23, 24$]$.

(2) Einige Bemerkungen über Singularitäten Taylorscher und Dirichletscher Reihen. Berliner Sitzungsberichte, I923, p. 39-44. [24].

(3) Über Potenzreihen, die überkonvergente Abschnittsfolgen besitzen.

Berliner Sitzungsberichte (1923) p. 185 - I92. [23, 24].

(4) Über allgemeine Konvergenzsätze des komplexen Funktionentheorie. Jahresbericht d. Deutschen Math.-Verein., t. 32 (1923) p. 185-194. [23, 24$]$.

(5) Über vollständige Gebiete gleichmässiger Konvergenz von Folgen analytischer Funktionen.

Hamburger Math. Abhandlungen, t. I (1923) p. $327-35^{\circ}$. [23, 24$]$.

(6) Über die Bedeutung der Jensenschen Formel für einige Fragen der komplexen Funktionentheorie. (Aus einem Briefe an Herrn F. Riesz.)

Acta Litt. ac. Sci., Szeged, t. I (1923) p. 80-87. [22].

(7) Über die Darstellung analytischer Funktionen durch Potenzreihen. Jahresbericht d. Deutschen Math.-Verein., t. 32 (I923) p. 286-295, et t. 34 (I925) p. $183 .[23,24]$.

(8) On Hadamard's test for singular points. Journal London Math. Soc., t. I (1926) p. 236--239. [22, 24$]$.

(9) On representation of analytical functions by power series. Journal London Math. Soc., t. I (I926) p. $25 \mathrm{I}-263$. [23, 24].

(1 o) Über einen Satz von Herrn Hadamard. Jahresbericht d. Deutschen Math.-Vẹrein., t. 35 (r926) p. I79-r82. [24].

(I I) Über Singularitäteu gewisser mit Lücken behafteten Potenzreihen. Jahresbericht d. Deutschen Math.-Verein., t. 35 (1926) p. 269-280. [24].

\section{B. Ottolenghi.}

(r) Somma generalizzata e grado di indeterminazione delle serie. Giornale di mat. di Battaglini, 3:e série, t. 2 (t. 49) (I 9 I I) p. 233-279. [2 2, 3I].

(2) Coesistenza e identità dei limiti di Hölder e di Cesàro. Padova, Prosperini, I9 I r, I6 p., $8^{\circ}$. [3 I $]$. 
Essai d'une bibliographie de la représentation analytique d'une fonction monogène.

\section{H. Padé.}

(1) Sur la possibilité de définir une fonction par une série entière divergente. Comptes Rendus, t. I I6 (27 Mars I 893) p. 686-687. [34].

(2) Sur les séries entières convergentes ou divergentes et les fractions continues rationnelles.

Acta Math., t. 18 (1894) p. 97-I I r. [34].

\section{P. Painlevé.}

(r) Sur le développement en série de polynômes d'une fonction holomorphe dans une aire quelconque. Comptes Rendus, t. Iо2 (22 Mars I 886) p. 672-675. [I3, I4].

(2) Sur les lignes singulières des fonctions analytiques. Thèse, Paris, I 887 ; aussi Annales de Toulouse, t. 2 (I888) p. B I-B I3o. $\left[\mathrm{I}_{2}, \mathrm{I}_{3}, \mathrm{I} 4\right]$.

(3) Sur la représentation des fonctions analytiques uniformes. Comptes Rendus, t. 126 (I 7 Janvier 1898) p. 200-202. [I3, I4].

(4) Sur le développement des fonctions uniformes ou holomorphes dans un domaine quelconque.

Comptes Rendus, t. I 26 (24 Janvier r 898) p. 318--32 I. [I 3, I 4].

(5) Sur le développement des fonctions analytiques pour les valeurs réelles des variables.

Comptes Rendus, t. I 26 (3 I Janvier r 898) p. $385-388$. [14].

(6) Sur le développement des fonctions réelles non analytiques. Comptes Rendus, t. I 26 (7 Février I 898) p. 459-46 I. [I 4 ].

(7) Sur le développement d'une branche uniforme de fonction analytique. Comptes Rendus, t. I 28 (23 Mai I 899) p. I 277 - 280 . [25].

(8) Sur le calcul des intégrales des équations différentielles par la méthode de Cauchy-Lipschitz.

Comptes Reudus, t. I28 (I9 Juin I899) p. I 505-т 508. [27].

(9) Sur le développement d'une branche uniforme de fonction analytique en série de polynomes.

Comptes Rendus, t. I 29 (3 Juillet 1899) p. $27^{--} 3^{\text {I. }}$ (25).

(ro) Sur le développement des fonctions analytiques de plusieurs variables. Comptes Rendus, t. I 29 ( I 0 Juillet r 899) p. 92-95. [ I 4, 25].

(I I) Notice sur les travaux scientifiques de M. Painlevé. Paris, Gauthier-Villars, $1900,{ }_{2} 8$ p., $4^{\circ}$. [ [ $\left.13,14,25\right]$.

( 2 ) Sur le développement des fonctions analytiques en série de polynomes. Comptes Rendus, t. I35 (7 Juillet I902) p. I I-I 5. [25].

(13) Observations sur la communication précédente. (Voir É. Borel n:o 30.) Comptes Rendus, t. I35 (2 I Juillet 1902) p. I $5^{2-153 .}$ [28].

8-2822. Acta mathematica. 52. Imprimó le 25 janvier 1929. 
(I4) Note I. Sur le développement des fonctions analytiques.

Dans Borel (E.): Leçons sur les fonctions de variables réelles et les développements en séries de polynomes (r905) p. IoI-I 48 . [I 4, 25$]$.

( 5 ) Observations au sujet de la communication précédente. (Voir A. Denjoy n:o r.) Comptes Rendus, t. I 48 (3 Mai I 909) p. I I 56- I I 57 . [13].

\section{G. Pedote}

(I) Sul concetto di prolungamento analitico.

Montepulciano, Della Querce, I9II, 22 p., $8^{\circ}$. [2 I].

\section{P. Peñalver y Bachiller.}

(r) Estudio elemental de prolongación analítica.

Thèse, Madrid, I9 I r, 68 p. [2 r, 25].

\section{O. Perron.}

(I) Die Lehre von den Kettenbrüchen. Leipzig \& Berlin, B. G. Teubner, I9I3, XII +520 p., $8^{\circ}$. [34].

(2) Über das infinitäre Verhalten der Koeffizienten einer gewissen Potenzreihe. Archiv d. Math. u. Phys., 3:e série, t. 22 (1914) p. 329-340. [22, 24, 35].

(3) Über die näherungsweise Berechnung von Funktionen grosser Zahlen. Münchener Sitzungsberichte, I9 I 7, p. I9 I-2 I9. [35].

(4) Zur Theorie der divergenten Reihen. Math. Zeitschrift, t. 6(I920) p. I58-I60. [3I].

(5) Beitrag zur Theorie der divergenten Reihen. Math. Zeitschrift, t. 6 (1920) p. 286-310. [25,3I].

(6) Neue Summationsmethoden und Entwicklungen nach Polynomen. Sitzungsberichte Heidelberger Akad., I922, N:0 I, I4 p. [25, 31].

(7) Über eine Verallgemeinerung der Eulerschen Reihentransformation.

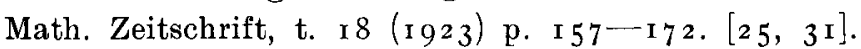

\section{P. Persson.}

(1) Recherches sur une classe de fonctions entières. Thèse, Upsal, I 908, 47 p. [32].

(2) Recherches sur une classe de fonctions entières de genre infini. Arkiv för Mat., Astr. o. Fys., t. 4, n:0 22 (I908) p. I-I6. [32].

\section{Petrovitch.}

(r) Un problème sur les séries.

Nouvelles Annales de Math., 3:e série, t. I 5 (r896) p. 58-63. [2 I].

(2) Remarque sur les zéros des séries de Taylor.

Bulletin Soc. Math. de France, t. 29 (I90I) p. 303-312. [24]. 
(3) Sur les fonctions représentées par une classe étendue d'intégrales définies. Bulletin Soc. Math. de France, t. 32 (1904) p. 67--103. [13, 24].

(4) Les spectres numériques.

Paris, Gauthier-Villars, I 9 I9, VIII + I to p., $8^{\circ} .[24]$.

\section{H. Petrini.}

(I) Sur une extension d'un théorème de M. Mittag-Leffler. Arkiv för Mat., Astr. o. Fys., t. I5, n:o 2 I (1921) 3 p. [12, I3].

\section{E. Phragmén}

( 1$)$ Sur une extension d'un théorème de M. Mittag-Leffler. Comptes Rendus, t. I 28 ( I 2 Juin I 899) p. 1434-I437. [25].

(2) Sur le domaine de convergence de l'intégrale infinie $\int_{0}^{\infty} F(a x) e^{-a} d a$. Comptes Rendus, t. I 32 (Io Juin I90I) p. I $396-$ I $399 .[25,3$ I]

(3) Sur une extension d'un théorème classique de la théorie des fonctions. Acta Math., t. 28 (I904) p. $35 \mathrm{I}-368$. [I3].

\section{E. Phragmén et E. Lindelöf.}

( 1 ) Sur une extension d'un principe classique de l'analyse et sur quelques propriétés des fonctions monogènes dans le voisinage d'un point singulier.

Acta Math., t. 3 I (1908) p. 38I-406. [13].

\section{É. Picard.}

(I) Mémoire sur les fonctions entières.

Annales de l'École Norm., 2 :e série, t. 9 (I880) p. I 45-I66. [I3].

(2) Sur la décomposition en facteurs primaires des fonctions uniformes ayant une ligne de points singuliers essentiels.

Comptes Rendus, t. 92 (2 I Mars I88I) p. 690-692. [I3].

(3) Sur les fonctions uniformes affectées de coupures. Comptes Rendus, t. 94 (22 Mai 1882) p. I405-1407. [13].

(4) Quelques remarques sur le prolongement des fonctions. Comptes Rendus, t. I 28 (23 Janvier i 899) p. 193-r95. [28].

(5) Sur le développement en séries des intégrales des équations différentielles par la méthode de Cauchy.

Comptes Rendus, t. I 28 (5 Juin 1899) p. I363-I366. [27].

(6) Sur certains développements en séries déduits de la méthode de Cauchy dans la théorie des équations différentielles ordinaires.

Annales de I'École Norm., 3:e série, t. 2I (I 904) p. I4I-I 5I. [25, 27 ].

(7) Sur le développement de l'analyse et ses rapports avec diverses sciences. Conférences faites en Amérique.

Paris, Gauthier-Villars, 1905, x67 p., $8^{\circ}$. [13, 25]. 


\section{S. Pincherle.}

(r) Alcuni teoromi sopra gli sviluppi in serie per funzioni analitiche. Rendiconti R. Ist. Lombardo, 2:e série, t. I5 (I882) p. $224-225$. [I4].

(2) Sopra un'applicazione della funzioni sferiche al theorema di Mittag-Leffler e alla determinazione delle funzioni a spazi lacunari.

Rendiconti d. Accad. d. Sci. d. Ist. d. Bologna, I 883, p. 100-105. [1 2, I3, I 4].

(3) Sui sistemi di funzioni analitiche e le serie formate coi medesimi. Annali di Mat., 2 :e série, t. I 2 (I883-I884) p. I I-4 I et Io7-I33. [I4].

(4) Sopra una formola del sig. Hermite.

Atti d. R. Accad. dei Lincei. Rendiconti, 4:e série, t. I (I 885 ) p. 267--268. [I I].

(5) Une formule dans la théorie des fonctions.

Öfversigt K. Vetenskapsakad. Förhandl., t. 43 (I 886) p. 5I-55. [I 2, I 4].

(6) Costruzione di nuove espressioni analitiche atte a rappresentare funzioni con un numero infinito di punti singolari.

Atti d. R. Accad. dei Lincei. Rendiconti, 4:e série, t. 3 ( 1887 ) p. $370-375$. $\left[I_{3}, I_{4}\right]$.

(7) Sur le développement d'une fonction analytique en série de polynômes.

Comptes Rendus, t. Io7 (I.7 Déc. I 888) p. 986-989. [I4].

(8) Sulle serie di potenze. (Estratto di una lettera al dottore Vivanti.)

Annali di Mat., 2:e série, t. 2 I (1893) p. I38-I40. [24].

(9) Sull'operazione aggiunta.

Rendiconti d. R. Accad. d. Sci. Bologna nuova serie, t. 2 (r897-98) p. r $30-$ I 39. $[24]$.

( 10$)$ Sulle singolarità di una funzione che dipende da due funzioni date.

Atti d. R. Accad. dei Lincei. Rendiconti, 5:e série, t. 8 (I899) p. $228-232$. [24].

(I I) A proposito di un recente teorema del Sig. Hadamard.

Rendiconti d. R. Accad. d. Scienze dell'Istituto di Bologna, t. 3 (I899) p. $67-74 .[24]$.

( 2 ) Di alcune operazioni atte ad aggiungere o togliere singolarità in una funzione analitica.

Annali di Mat., 3:e série, t. 4 (1900). p. 2 19-280. [24]

(13) $\mathrm{Di}$ una nuova operazione funzionale e di qualche sua applicazione.

Rendiconto R. Accad. d. Sci., Bologna, nuova série, t. 7 (1903) p. 83-98. $[22,24]$.

(14) Sugli sviluppo assintotici e le serie sommabili.

Atti R. Accad. Naz. dei Lincei. Rendiconti, 5:e série, t. I3 (I904) p. 5 I3-5 I9. $[3$ I, 34].

( 5 ) Sur les fonctions déterminantes.

Annales de l'École Normale, 3:e série, t. 22 (1905) p. 9-68. [24].

( 6 ) Sulle singolarità di una funzione che dipende da due funzioni date.

Atti d. R. Accad. dei Lincei, Rondiconti, 5:e série, t. I 5 (1906) p.603-6 ro. [24]. 
Essai d'une bibliographie de la représentation analy tique d'une fonction monogène. 61

(I7) Sopra l'estensione agli sviluppi assintotici di un teorema del sig. Hurwitz. Atti d. R. Áccad. dei Lincei, Rendiconti, 5:e série, t. I6 (1907) p. 3-5. [24,34].

( 8 ) Alcune spigolature nel campo delle funzioni determinanti. Atti del IV Congresso int. dei mat. Roma r9o8, t. 2, p. 44-48. [24].

( 19$)$ Sulle funzioni trascendenti semplici. Atti R. Accad. Naz. dei Lincei, Rendiconti, 5:e série, t. 33: I (1924) p. 52-57. [24].

(20) Ancora sulle funzioni trascendenti semplici.

Atti R. Accad. dei Lincei, Rendiconti, 5:e série, t. 33: x (1924) p. 203-206. [24].

(2 I) Su una separazione di singularità in una funzione analitica.

Atti R. Accad. Naz. dei Lincei, Rendiconti, 5:e série, t. 33, I:er semestre (1 924) p. $249-253$. [24].

\section{S. Pincherle et U. Amaldi.}

(r) Le operazioni distributive e le loro applicazioni all'analisi. Bologna, Zanichelli, I 90 I, XII +490 p., $8^{\circ}$. [24].

\section{G. Pirondini.}

( $)$ Nuovo metodo per costruire delle funzioni continue a spazi lacunari. Parma, Rossi-Ubaldi, r 905, r 6 p., $8^{\circ}$. [ I 2 ].

\section{Plancherel.}

(I) Sur la sommation des séries de Laplace et de Legendre. Rendiconti Circ. Mat. di Palermo, t. 33 (I912) p. 4[-66. [3I] .

(2) Le développement de la théorie des séries trigonométriques dans le dernier quart de siècle.

L'enseignement math., t. $24(1924-25)$ p. I9-58. [22, 31]

\section{A. Plessner.}

(1) Zur Theorie der konjugierten trigonometrischen Reihen. Mitteilungen des Math. Sem. d. Universität Giessen, X. Thèse, Giessen, 1923, 36 p., $8^{\circ}$. [22].

\section{H. Poincaré.}

(1) Sur les séries des polynômes. Comptes Rendus, t. 96 (5 Mars 1883) p. 637-639. [14].

(2) Sur les fonctions à espaces lacunaires. Comptes Rendus, t. 96 ( 6 Avril r883) p. I I34- I 36 . [1 2].

(3) Sur les fonctions à espaces lacunaires. Acta Soc. Sci. Fennicae, t. I 2 (1 883 ) p. 34 I-350. [ 2 ].

(4) Sur les équations linéaires aux différentielles ordinaires et aux différences finies. American Journal of Math., t. 7 (1885) p. $203^{-2} 5^{8}$. [I4]. 
(5) Sur les intégrales irrégulières des équations linéaires. Acta Math., t. 8 (1886) p. 295-344. [34].

(6) Sur les fonctions à espaces lacunaires.

American Journal of Math., t. I4 (I892) p. $20 \mathrm{I}-22 \mathrm{I}$. [r2].

\section{G. Pólya.}

(I) Une série de puissances est-elle en général non-continuable? L'enseignement math, t. I 7 (I9I5) p. 343-344. [23].

(2) Über Potenzreihen mit ganzzahligen Koeffizienten. Math. Annalen, t. 77 (I9I6) p. 497-5I3. [22, 23].

(3) Über Potenzreihen mit endlich vielen verschiedenen Koeffizienten. Math. Annalen, t. 78 (19 I 7 ) p. 286-293. [24].

(4) Über die Potenzreihen, deren Konvergenzkreis natürliche Grenze ist. Acta Math., t. 4 I (1917) p. 99-I I 8. [23].

(5) Sur les propriétés arithmétiques des séries entières, qui représentent des fonctions rationnelles.

L'enseignement math., t. I9 (I9I7) p. 323. [24].

(6) Arithmetische Eigenschaften der Reihenentwicklungen rationaler Funktionen. Journal für Math., t. I 5 I (I920) p. I-3 I. [24].

(7) Bemerkung über die Mittag-Lefflerschen Funktionen $E_{\alpha}(z)$. (Auszug aus einem an Herrn Mittag-Leffler gerichteten Briefe.) Tôhuku Math. Journal, t. I9 (I92I) p. $24 \mathrm{I}-248$. [32].

(8) Sur les séries entières à coefficients entiers. Proceedings London Math. Soc., série 2, t. 2 I (1922) p. 22-38. [23, 24$]$.

(9) Arithmetische Eigenschaften und analytischer Charakter.

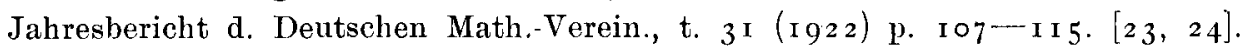

(10) Ueber die Existenz unendlich vieler singulärer Punkte auf der Konvergenzgeraden gewisser Dirichletscher Reihen.

Berliner Sitzungsberichte, I923, p. $45-50$. [2 2 ].

(I I) Analytische Fortsetzung und konvexe Kurven. Math. Annalen, t. $89\left(\right.$ I $\left._{23} 3\right)$ p. I 79-19I. $[24,25]$.

(1 2) Sur l'existence d'une limite considérée par M. Hadamard. L'enseignement math., t. 24 (1924-25) p. $76-78$. [24].

\section{Pompéiu.}

(I) Sur les fonctions de variables complexes. Comptes Rendus, t. I34 (26 Mai I902) p. I I95-II97. [I3, I6].

(2) Sur les singularités des fonctions analytiques uniformes. Comptes Rendus, t. I39 (28 Nov. I 904) p. 9I4-9I5. [I3].

(3) Sur la continuité des fonctions de variables complexes. Annales de Toulouse, série 2, t. 7 (1905) p. 264-3 I5. Publié aussi comme Thèse, Paris, Gauthier-Villars. [I3]. 
Essai d'une bibliographie de la représentation analytique d'une fonction monogène.

(4) Sur les singularités des fonctions analytiques uniformes.

Comptes Rendus, t. I49 (I 2 Juillet I 909) p. I03- I05 et t. I 50 (2 I Février I 9 IO) p. $454-455$. [I3].

(5) Sur les singularités discontinues des fonctions analytiques uniformes. Comptes Rendus, t. I 49 (6 Déc. I 909) p. I050-I05I. [I3].

(6) Sur la représentation des fonctions analytiques par des intégrales définjes. Comptes Rendus, t. I 49 (27 Déc. r 909) p. I355- I357. [13].

(7) Sur les singularités des fonctions analytiques uniformes. Rendiconti Circ. Mat. di Palermo, t. 29 (1910) p. 306-307. [13].

(8) Sur un exemple de fonction analytique partout continue. American Journal of Math., t. 32 (rgro) p. 327-332. [r3].

(9) Sur les singularités des fonctions analytiques uniformes. L'enseignement math., t. I 2 (1910) p. 18-20. [13].

(10) Sur les fonctions analytiques uniformes. Nieuw Archief voor Wiskunde (2) 9 (I9IO) I72-175. [I3].

(I I) Sur les fonctions de variable complexe. Comptes Rendus, t. I 53 (2 Oct. I9II) 624-626. [13].

( 2 ) Sur certaines séries de fractions simples. Nieuw Archief voor Wiskunde, série 2, t. io (I9 I3) p. 353-356. [ I I, I4].

(13) Sur les polynomes d'approximation.

Tôhuku Math. Journal, t. 6 ( ( 9 I 4 ) p. I I $\left.5^{- \text {I I } 9 . ~[~[~} 5\right]$.

\section{K. Popoff.}

(1) Sur le développement d'une fonction holomorphe en série de polynomes et de fractions rationnelles.

Bulletin Soc. Math. de France, t. 52 (x923) p. 532-536. [13, I4].

(2) Sur les lacunes que peut présenter une série de Taylor qui représente une fonetion analytique régulière à l'infini et qui n'admet qu'un point singulier qui est un point singulier essentiel.

Comptes Rendus, t. I 82 (8 Février 1926) p. 369-37 I. [24].

\section{B. Porter.}

(I) The polynomial convergents of a power series. Annals of Math., série 2, t. 8 (I907) p. I $89-$ I92. [I I, 23, 24 ].

\section{K. A. Poukka.}

(x) Über die Cauchy'schen reziproken Funktionen und deren Anwendung auf die analytische Fortsetzung der Taylor'schen Reihe. Thèse, Helsingfors $1907,1 \mathrm{~V}+49 \mathrm{p} ., 8^{\circ} .[24,25]$.

(2) Die analytische Fortsetzung von Potenzreihen, deren Koeffizienten durch ganze Funktionen der Ordnungszahl ausgedrückt werden können.

Annales Acad. Sci. Fennicae (A), t. 7, n:o I 5 (1916) I6 p. [24]. 


\section{A. Pringsheim.}

(r) Ueber gewisse Reihen, welche in getrennten Convergenzgebieten verschiedene, willkürlich vorgeschriebene Functionen darstellen.

Math. Annalen, t. 22 (1883) p. io9-i i6. [I i ].

(2) Ueber das Verhalten gewisser Potenzreihen auf dem Convergenzkreise.

Math. Annalen, t. 25 (1885) p. 419-426. [22].

(3) Zur Theorie der Taylor'schen Reihe und der analytischen Funktionen mit beschränktem Existenzbereich.

Münchener Berichte, t. 22 (1892) p. 2 I I--245. [1 2, 23$]$.

(4) Zur Theorie der Taylor'schen Reihe und der analytischen Functionen mit beschränktem Existenzbereich.

Math. Annalen, t. 42 (1 893 ) p. I 53-184. [I 2, 23$]$.

(5) Ueber Functionen, welche in gewissen Punkten endliche Differentialquotienten jeder endlichen Ordnung, aber keine Taylor'sche Reihenentwickelung besitzen. Math. Annalen, t. $44(1894)$ p. $4 \mathbf{I}^{-}-56$. [22].

(6) Ueber die nothwendigen und hinreichenden Bedingungen des 'Taylor'schen Lehrsatzes für Functionen einer reellen Variabeln.

Math. Annalen, t. 44 (1894) p. 57-82. [23].

(7) Ueber Potenzreihen auf dem Convergenzkreise und Fourier'sche Reihen.

Sitzungsberichte .... München, t. 25 (1895) p. 337-364. [22].

(8) Ueber zwei Abel'sche Sätze, die Stetigkeit von Reihensummen betreffend.

Münchener Berichte, t. 27 (1897) p. 343-356. [22].

(9) Ueber eine besondere Gattung von singulären Stellen analytischer Functionen. Math. Annalen, t. $50(1898)$ p. $442-46$ I. [1 4, 24$]$.

(ro) Irrationalzahlen und Konvergenz unendlicher Prozesse. Encyklopädie d. Math. Wissenschaften IA3, 1898, p. $47 \rightarrow{ }_{4} 6$. [30].

(x 1) Ueber das Verhalten von Potenzreihen auf dem Convergenzkreise. Sitzungsberichte .... München, t. 30 (1900) p. 37-100. [2 2].

(1 2) Über die Divergenz gewisser Potenzreihen an der Convergenzgrenze. Sitzungsberichte .... München, t. 3 I (190I) p. 505-524. [22].

(r3) Über den Divergenz-Charakter gewisser Potenzreihen an der Convergenzgrenze. Acta Math., t. 28 (1904) p. I-3o. [22].

(14) Über einige funktionentheoretische Anwendungen der Eulerschen Reihen-Transformation.

Münchener Berichte, I9t2, p. II-9r. [22, 23, 24, 25, 3I].

( 5 ) Über die Äquivalenz der sogenannten Hölderschen und Cesàroschen Grenzwerte und die Verallgemeinerung eines beim Beweise benützten Grenzwertsatzes.

Münchener Berichte, I9I6, p. 209-224 et I9 18, p. 89-92. [3I].

(r6) Über eine Konvergenzbedingung für unendliche Reihen, die durch iterierte Mittelbildung reduzibel sind.

Münchener Sitzungsberichte, I 920 , p. $275-284$. [33]. 
(r) Sur les fonctions conjuguées.

\section{Privaloff.}

Bulletin Soc. Math. de France, t. 44 (r916) p. roo-то3. [22].

(2) Sur la convergence des séries trigonométriques conjuguées. Comptes Rendus, t. I65 (I6 Juillet I9I7) p. 96-99. [22, 31].

(3) Integral Cauchy. [= L'intégral de Cauchy.] Izvjestij Saratovskavo Universiteta Fiz.-Mat. Fak. I9I8. Thèse, 94 + II p. [22].

(4) K teorii soprjažennych trigonometričeskich rjadov. [= Sur les séries trigonométriques conjuguées.]

Mat. Sbornik izd. Moskovskim Mat. Obščestvom, t. 3 I (I923) p. 224-228. [22].

(5) $\mathrm{Ob}$ obščenie teoremy Fatou. [= Sur une généralisation d'un théorème de Fatou.] Mat. Sbornik izd. Moskovskim Mat. Obščestvom, t. 3I (I9z3) p. 232-235. [22].

(6) O rjade Taylora. [-Sur la série de Taylor.] Mat. Sbornik, t. 3 I (I924) p. 345-349. [22, 33].

(7) $\mathrm{Ob}$ odnom processe summirovanija beskonečnich rjadov. [=Sur une méthode nouvelle de sommation des séries infinies.]

Mat. Sbornik, t. 32 (1925), p. 7 I I-722. [3 I].

(8) Sur certaines propriétés métriques des fonctions analytiques. Comptes Rendus, t. I 78 (I I Février r 924 ) p. 6I I-6I4. [22].

\section{A. P. Pšeborskij.}

(I) Sur les fonctions lacunaires. Notion d'une fonction lacunaire. Démonstration d'un théorème de $M$. Goursat (en russe).

Kievskija Univers. Izvestija, I 897 , n:o 7 , p. 49-6o. [I 2].

(2) Izslĕdovanija po teorii analitičeskich funkcij. Zadača o prodolženii rjada Taylora. [= Recherches sur la théorie des fonctions analytiques. Le problème du prolongement d'une série de Taylor.] Charkov, I 908, p. IV $+\mathrm{I}_{3} 8.8^{\circ}$. [25].

\section{J. von Puzyna.}

(r) Ueber eine methodische Bildung der analytischen Ausdrücke $\boldsymbol{\Sigma} f_{v}(x), \mathbf{\Sigma} f_{v}(x, y)$ von constanten Werten.

Monatshefte für Math. und Physik, t. 5 (I 894 ) p. 67--84. [II].

(2) Do teoryi szeregów potęgowych. [= Sur la théorie des séries entières.]

Kraków Akad. (Mat.-Przyrod.) Rozprawy., z:e série, t. i I (1897) p. 270-289. Bulletin Internat. Acad. Sci. de Cracovie, I896, p. 295-296. [22].

(3) Über Summen unendlich vieler Potenzreihen und über die Funktionentheoretischen Sätze des Herrn Mittag-Leffler.

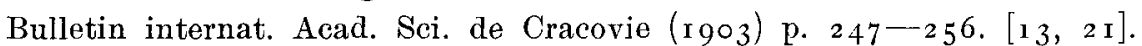

\section{F. G. Radelfinger}

(I) Analytic representation of complex functions.

Bulletin Philosophical Soc. Washington, t. I4 (Ig02) p. $227-232$. [25].

9-2822. Acta mathematica. 52. Imprimé le 25 janvier 1929. 


\section{H. Rademacher.}

(r) Über den Konvergenzbereich der Eulerschen Reihentransformation. Sitzungsberichte d. Berliner Math. Gesellschaft, I922, p. 16--24. $[25,31]$.

\section{J. Rémoundos.}

(I) Les singularités des équation différentielles et les séries sommables. Bulletin Soc. Math. de France, t. 48 (1920) p. 95- io6. [27, 3 I]

\section{F. Renaux.}

(I) Sur un développement d'une fonction holomorphe à l'intérieur d'un contour en une série de polynômes.

Comptes Rendus, t. I 29 (1 8 Sept. I 899) p. 473-475. [14].

(2) Sur les fonctions fondamentales et sur le développement d'une fonction holomorphe à l'intérieur d'un contour en série de fonctions fondamentales.

Comptes Rendus, t. I 29 (9 Oct. I 899) p. 545-548. [i 4 ].

\section{E. Ricotti.}

(I) Sulle serie divergenti sommabili.

Giornale di mat. di Battaglini, t. 48 (ou série 3, t. I) (I9 Io) p. 79-I I I. [3 r]

\section{K. Rieder.}

(I) Polynomische Entwicklungen von Funktionen einer komplexen Variabeln. Thèse, Basel, I 9 r r, 85 p., $8^{\circ}$. [I 3, I 4,25$]$.

\section{F. Riesz.}

(1) Über die Randwerte einer analytischen Funktion.

Math. Zeitschrift, t. I $8\left(\lg _{92} 3\right)$ p. $87-95$. [22].

\section{F. et M. Riesz.}

(I) Über die Randwerte einer analytischen Funktion.

Compte rendu du quatrième congrès des Math. Scand. à Stockholm (1916) p. 27 $-44 .[22]$.

\section{F. Riesz et G. Szegö.}

(1) Analytikus függvények kerületi értékeiröl. [ = Sur les valeurs limites de fonctions analytiques.]

Math. és termész. értesitô, t. $3^{8}$ (1921) p. I I 3 -1 27 . [22].

\section{Riesz.}

(1) Megadott hatványsor folytatásának analitikai eloállitása. [=- La représentation analytique du prolongement d'une série entière donnée.]

Math. és Phys. lapok, t. r6 (r907) p. r-25; t. I7 (r9o8) p. 96-ro8. [25]. 
Essai d'une bibliographie de la représentation analytique d'une fonction monogène.

(2) A hatványsor összegezhetősége az összetartási körön. [= Sur la sommabilité des séries entières sur leur cercle de convergence.]

Math. és termész. értesitô, t. 26 (1908) p. 22 I-229. [22].

(3) Összegezheto" trigonometrikus sorok és összegezhetó hatványsorok. Doktori értekezés. [= Séries trigonométriques et séries entières sommables. Thèse.]

Budapest, I908, Math. és phys. lapok, t. I 9 (I9 Io) p. I- 56 . [22, 3I, 33].

(4) Sur les séries de Dirichlet.

Comptes Rendus, t. I 48 (2 I Juin I909) p. r658-1660. [3 г].

(5) Sur la sommation des séries de Dirichlet.

Comptes Rendus, t. I49 (3 Juillet I909) p. I8-2 I. [3 I].

(6) Sur les séries de Dirichlet et les séries entières.

Comptes Rendus, t. I 49 (22 Nov. r909) p. 909-9 I 2. [2 2, 31, 33].

(7) Sur un problème d'Abel. (Extrait de deux lettres à M. G. Mittag-Leffler.)

Rendiconti Cire. Mat. di Palermo, t. 30 (r9ro) p. 339-345. [26].

(8) Une méthode de sommation équivalente à la méthode des moyennes arithmétiques.

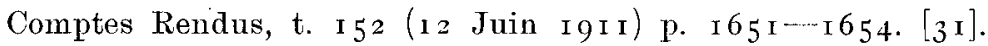

(9) Über einen Satz des Herrn Fatou.

Journal für Math., t. I 40 (I 9 I I) p. 89-99. [2 2, 33].

( $о$ o) Représentation du prolongement analytique d'une série de Dirichlet (en hongrois). Math. és termész. értésito, t. 29 ( I 9 I I ) p. $283^{-3}$ - 0 I. [25].

(I I) Sur la représentation analytique des fonctions définies par des séries de Dirichlet.

Acta Math., t. 35 (1912) p. 253-270. [25].

(1 2) Neuer Beweis des Fatouschen Satzes.

Göttinger Nachrichten, I 9 I6, p. 62--65. [22, 33].

(I3) Sätze über Potenzreihen.

Arkiv f. Mat., Astr. o. Fys., t. I I, n:o I 2 (I916) I 6 p. $[22,33]$.

( 14$)$ Sur l'équivalence de certaines méthodes de sommation.

Proceedings London Math. Soc., 2:e série, t. 22 (I924) p. 4I2-4Ig. [3I].

\section{J. F. Ritt.}

(I) On the derivatives of a function at a point.

Annals of Math., sér. 2, t. I8 (1916) p. 18-23. [13].

\section{Runge.}

( 1 ) Zur Theorie der eindeutigen analytischen Functionen.

Acta Math., t. 6 (1884) p. 229-244. [I3, r 4 ].

(2) Zur Theorie der analytischen Functionen.

Acta Math., t. 6 (1885) p. $245^{-2}{ }_{4} 8$. [I 4 ]. 
(3) Über empirische Funktionen und die Interpolation zwischen äquidistanten Ordinaten.

Zeitschrift fïr Math. und Physik, t. 46 (I90I) p. 224-243. [I4, I 5].

\section{G. Sannia.}

(I) Nuova trattazione del metodo di Borel per la sommazione delle serie. Atti R. Accad. di Torino, t. 52 (1916-17) p. 67-86. [31].

(2) Nuovo metodo di sommazione delle serie: estensione del metodo di Borel. Rendiconti Circ. Mat. di Palermo, t. 42 (I9I7) p. 303-322. [3I].

(3) Sul metodo di Borel per la sommazione delle serie. Atti Accad. dei Lincei. Rendiconti, 5:e série, t. 26: 1 (I917) p. 162-167. [31].

(4) Generalizzazione del metodo di Borel per la sommazione delle serie. Atti Accad. dei Lincei. Rendiconti, 5:e série, t. 26: I (I9I7) p. 603-606. [31].

(5) Le serie di funzioni sommate col metodo di Borel generalizzato. Atti Accad. dei Lincei. Rendiconti, 5:e série, t. 26:2 (I9I7) p. 77-8I. [3 I].

(6) Le serie di potenze di una variabile sommate col metodo di Borel generalizzato. (Nota I, II.)

Atti R. Accad. di Torino, t. 53 (I9I7-18) p. I35-148, 192-205. $[25,3 \mathrm{I}]$.

(7) Sulle serie di potenze di una variabile sommate col metodo di Borel generalizzato. Nota I, II, III. (Les mots »di una variabile» sont supprimés dans le titre de la 2 :ème note.)

Atti R. Accad. Naz. dei Lincei. Rendiconti, 5:e série, t. 27: I (1918) p. 98I 02, I $39-$ I 42 et t. $27: 2$ (I 9 I 8) p. $24-27$. [25,3 I]

(8) Il metodo di sommazione di Eulero e la moltiplicazione delle serie.

Atti R. Accad. Naz. dei Lincei. Rendiconti, 5:e série, t. 27:2 (I9I8) p. $397-$ 399. $[22,3 \mathrm{I}]$.

(9) Estensione e studio di un metodo di sommazione generico di Borel.

Scritti mat. offerti ad Enrico d'Ovidio (I9r8) p. 227-252. [3I].

(ro) Serie di funzioni sommabili uniformemente col metodo di Borel generalizzato. Atti R. Accad. di Torino, t. 54 (I9I8-r9) p. I7I-I85 et t. 55 (I9I9--20) p. 310-322. [3 I ].

( I I) Risoluzione dell'equazione di Fredholm con serie assolutamente sommabili del Borel.

Atti R. Accad. Naz. dei Lincei. Rendiconti, 5:e série, t. 28: 2 (1919) p. 429433. $[27,3 \mathrm{I}]$.

(12) Sviluppo di una funzione analitica in serie sommabili col metodo di Borel. Rendiconto Accad. scienze fis. e mat. di Napoli, série 3, t. 25 ( I9 I9) p. I 25 - I 37. [ I 3, I 4, 25, 3 I] .

(13) Nuovo metodo di sommazione delle serie che ammette l'algoritmo delle serie assolutamente convergenti.

Atti Accad. dei Lincei. Rendiconti, 5:e série, t. 29: I (I920) p. I4I-I 46. [3I]. 
Essai d'une bibliographie de la représentation analytique d'une fonction monogène.

(14) Serie assolutamente sommabili col metodo di Borel generalizzato. Atti R. Accad. di Torino, t. 56 (I92x) p. $34-40$. [31].

\section{E. Schering.}

(I) Das Anschliessen einer Function an algebraischen Functionen in unendlich vielen Stellen.

Abhandl. K. Ges. d. Wiss. zu Göttingen, t. 27 (1880) 64 p. [I3].

\section{F. Schimpf.}

(I) Eine Theorie der Konvergenz unendlicher Reihen. Programme n:o 353, Gymn. Bochum, 1895 , I I- 56 p., $4^{\circ}$. [22].

(2) Zur Definition der Convergenz der unendlichen Reihen und der unendlichen Producte. Mehrfache Grenzgleichungen. Grenzgleichungen periodischer Reihen. Programme n:o 364, Gymn. Bochum, I 898,30 p., 4․ [22].

\section{R. Schmidt.}

(r) Über divergente Folgen und lineare Mittelbildungen.

Math. Zeitschrift, t. $22\left(\right.$ I925 $^{2}$ p. $89^{-}$I $^{2}$. Thèse, Kiel, I $923 .[3$ I, 33].

\section{W. Schnee.}

( I) Die Identität des Cesàroschen und Hölderschen Grenzwertes. Math. Annalen, t. $67(1909)$ p. I Io-I 25. [3 I ].

\section{Else Schöll.}

(r) Beiträge zur Theorie der analytischen Fortsetzung in elementarer Behandlungsweise.

Inaugural-Dissertation. München, I 9 I 4, 88 p., $8^{\circ}$. [24, 25, 31].

\section{Schur.}

(I) Über die Äquivalenz der Cesàroschen und Hölderschen Mittelwerte. Math. Annalen, t. 74 (I9I3) p. $447-45^{8}$. [30, 3 I].

(2) Über lineare Transformationen in der Theorie der unendlichen Reihen. Journal für Math., t. r 5 (I920) p. 79-I Ir. [30].

\section{R. Schwarz.}

(1) Der Eisensteinsche Satz über die Koeffizienten der Reihenentwicklungen algebraischer Funktionen.

Thèse, Tübingen, I $908,30 \mathrm{p} ., 8^{\circ}$. [24].

\section{Servant.}

(I) Sur les points singuliers d'une fonction définie par une série de Taylor.

Comptes Rendus, t. I 28 (9 Janvier I 899) p. 80-83. [22, 24, 26]. 
(2) Essai sur les séries divergentes.

Annales de Toulouse, série i, t. I (I899) p. I I7-I75. Aussi Thèse, Paris, Gauthier-Villars. $[24,25,26,3 \mathrm{I}]$.

\section{Severini.}

(i) Sul raggio di convergenza delle serie di potenze.

Bolletino dell'Accad. Gionia di Sci. Nat. in Catania. Fasc. 94 (r907) z p. [2 I].

\section{F. Sibirani.}

(I) Un teorema della teoria delle serie di potenze. Jornal de Sci. Math. e Astr., t. I5 (1903) p. 79--84. [23].

(2) Su la rappresentazione approssimata di una funzione continua di variabile reale o complessa per combinazioni lineari di assegnate funzioni. Rendiconti Circ. Mat. di Palermo, t. 34 (19 r 2) p. I $32^{-}$I 57 . [I 5 ].

(3) Espressioni analitiche che definiscono più funzioni analitiche ad area lacunare. Atti R. Accad. Sci. di Torino, t. 55 (I9I9-20) p. 46-50. [I I, I 2].

\section{Siegel.}

(1) Über die Coeffizienten in der Taylorschen Entwicklung rationaler Functionen. Tôhuku Math. Journal, t. 20 (1921) p. 26-31. [24].

\section{W. Sierpiński.}

(I) Sur une série potentielle qui, étant convergente en tout point de son cercle de convergence, représente sur ce cercle une fonction discontinue. Rendiconti Circ. Mat. di Palermo, t. 4 I (I9I6) p. i87-igo. [22].

(2) 0 szèregu potęgowym który jest zbiezny na całem swem kole zbiezności jednostajnie ale nie bezwzględnie. [ - Sur une série de puissances convergente uniformement mais jamais absolument sur tout le cercle de convergence.] Prace mat.-fiz., t. 29 (19i8) p. $263-266$. [22].

\section{L. Silverman.}

(r) On the definition of the sum of a divergent series. Thèse, Univ. of Missouri Studies, Math. series, t. I, n:o r ( 9 I 3 ) 96 p. [3o].

(2) The equivalence of certain regular transformations. Transactions Amer. Math. Soc., t. 26 (I924) p. IoI-II2. [3I].

\section{V. Slešinski.}

(I) $\mathrm{K}$ teorii analitičeskich funkeij. [= Sur la théorie de fonctions analytiques.] Mat. Sbornik izd. Moskovskim Mat. Obščestrom, t. I7 (1893) p. 223-228. [2 2]. 
Essai d'une bibliographie de la représentation analytique d'une fonction monogène.

\section{L1. L. Smail.}

(1) Some generalizations in the theory of summable divergent series. Thèse, Columbia, r 9 r 3. [3o].

(2) A general method of summation of divergent series. Annals of Math., (2) 20 (I 918 ) I 49-I 54. [30, 3 I ].

(3) Elements of the theory of infinite processes. New York, Mc Graw-Hill, 1923, 336 p. [30].

(4) History and synopsis of the theory of summable infinite processes. University of Oregon Publication, t. 2, n:o 8 (I925) VI I I75 p. [3o].

\section{Th. Soubbotine.}

(I) $\mathrm{Ob}$ opredělenii osobych toček analitičeskoj funkcij. [= Sur la détermination des points singuliers d'une fonction analytique.]

Mat. Sbornik izd. Moskovskim Mat. Obščestrom, t. 3o (1916) p. 402-433. [24, $25,26]$.

\section{Soudée.}

( $\mathrm{x}$ ) Solution de la question I624 (Appell).

Nouvelles Annales de Math., (3) I 2 (1893) p. 4*-6*. [22].

\section{J. Soula.}

( I) Généralisation d'un théorème de $M$. Leau relatif à la recherche des points singuliers d'une fonction définie par une série de Taylor. Comptes Rendus, t. I 7 I (4 Oct. I920) p. 6I4-6i6. [24].

(2) Sur la recherche des points singuliers de certaines fonctions définies par leur développement de Taylor.

Journal de Math., série 8, t. 4 (I92I) p. 97--153. Aussi Thèse, Paris, GauthierVillars. $[23,24]$

(3) Sur la »séparation» des points singuliers d'une fonction analytique. Journal de Math., série 9, t. i (1922) p. 85-93. [24].

(4) Sur les séries de Taylor qui ont une infinité de coefficients nuls. Comptes Rendus, t. I 76 (26 Mars 1923) p. $877-879$. [24].

(5) Sur les fonctions analytiques qui s'annulent pour les valeurs entières et positives de la variable.

Comptes Rendus, t. I8I (26 Oct. I925) p. 538-539. [24].

\section{Spineanu.}

(I) Sur le développement d'une fonction holomorphe en série d'inverses de poly. nomes et en série de fractions rationnelles.

Comptes Rendus, t. I59 (27 Juillet I9 I4) p. 299-30 . [I3, I 4]. 


\section{P. Stäckel.}

(r) Zur Theorie der eindeutigen Functionen.

Journal für Math., t. Io6 (I890) p. I 89-i 92 . [I 3].

(2) Zur Theorie der eindeutigen analytischen Functionen.

Journal für Math., t. I I 2 (I893) p. 262-264. [I I, I 2, I3, 23].

\section{H. Steinhaus.}

(1) Kilka słów o uogólnieniu pojęcia granicy. [= Quelques remarques sur la généralisation de la notion de limite.] Prace mat.fiz., t. 22 (I9II) p. I 2 I-I34. [30].

(2) O pewnym szeregu potęgowym przedstawiajacym na kole zbiezności funkcyę pantachicznie nieciagła. [= Sur une série entière qui représente une fonction dont les discontinuités sont partout sur le cercle de convergence.]

Sprawozdania $z$ posiedzen Towarzystwo Naukowego [= Comptes Rendus de la Soc. sci. de Varsovie], t. 6 (I9I3) p. 356-367. [23].

(3) Sur un problème de MM. Lusin et Sierpiński.

Bulletin internat. de l'Acad. des Sciences de Cracovie. A. I9 I3, p. 435-450. [2 2].

(4) O pewnym szeregu Pringsheima. [= Sur une série de M. Pringsheim.]

Bulletin int. Acad. Sci. de Cracovie, 1918, p. I53-160. [22].

\section{T. J. Stieltjes.}

(I) Recherches sur quelques séries semi-convergentes.

Annales de l'École Norm., 3:e série, t. 3 (I886) p. 20I--258. Aussi comme Thèse, (Euvres complètes, t. 2 ( I 9 I 8) p. 2-58. [34].

(2) Sur les séries qui procèdent suivant les puissances d'une variable.

Comptes Rendus, t. I०3 (20 Déc. I 886) p. I 243-1246. Euvres complètes, t. 2 (1918) p. 69-72. [22].

(3) Sur la réduction en fraction continue d'une série procédant suivant les puissances descendantes d'une variable.

Annales Fac. Sci. de Toulouse, t. 3 (1889) p. H. r-I 7. (Euvres complètes, t. 2 ( 9 I 8 ) p. I $84-200$. [34].

(4) Exemple d'une fonction qui n'existe qu'à l'intérieur d'un cercle.

Bulletin Sciences Math., série 2, t. I I ( I 887) p. 46-5i. Euvres complètes, t. 2 (1918) p. 89-94. [12, 23].

(5) Sur le développement de $\log \Gamma(a)$.

Journal de Math., 4:e série, t. 5 ( I 889) p. 425-444. Euvres complètes, t. 2 (1918) p. 2 I I-230. [34].

(6) Recherches sur les fractions continues.

Annales Fac. Sci. de Toulouse, t. 8 (1894) p. J. I-r 22 et t. 9 (I895) p. A. 547. Euvres complètes, t. 2 (I 9 I 8 ) p. 402-- 566 . [34]. 
Essai d'une bibliographie de la représentation analytique d'une fonction monogène. 73

\section{O. Stolz.}

(1) Beweis einiger Sätze über Potenzreihen.

Zeitschrift für Math. und Physik, t. $20(1875)$ p. $369-376$. Addition t. 29 (1884) p. $127-128$. [22].

\section{H. Stone.}

(1) On the order of an analytic function at a singular point. Annals of Math., 2 :e série, t. 26 (1924) p. I 45-1 54. [22].

(2) The Borel summability of Fourier Series. American Journal of Math., t. 48 (I926) p. I O I-I I 2. [3 I].

(3) A comparison of the series of Fourier and Birkhoff. Trans. Amer. Math. Soc., t. 28 (I926) p. 695-76I. Thèse, Harvard Univ. [3I].

\section{E. Study.}

(I) Einige elementare Bemerkungen über den Prozess der analytischen Fortsetzung. Math. Annalen, t. 63 (I907) p. 239-245. [2 I, 25].

\section{B. Svension.}

(I) Études sur les fonctions définies par une série de Taylor.

Dissertation. Lund, r 908,99 p., $8^{\circ} .[24,25]$.

\section{O. Szász.}

(I) Über arithmetische Eigenschaften gewisser uvendlichen Zahlenfolgen und zugehöriger Potenzreihen.

Archiv d. Math. u. Phys., 3:e série, t. 26 (1917) p. 125-132. [24].

(2) Über Potenzreihen, die im Einheitskreise beschränkte Funktionen darstellen. Math. Zeitschrift, t. 8 (r920) p. $222-236$. [22].

(3) Über Singularitäten von Potenzreihen und Dirichletschen Reihen am Rande des Konvergenzbereiches.

Math. Annalen, t. 85 (1922) p. 99-1 10. [22].

\section{G. Szegö.}

(I) Über Potenzreihen, deren Koeffizienten zahlentheoretische Funktionen sind. Math. Zeitschrift, t. 8 (I920) p. 36-5 I et t. 2 I (1924) p. 208. [23].

(2) Über orthogonale Polynome, die zu einer gegebenen Kurve der komplexen Ebene gehören.

Math. Zeitschrift, t. 9 (I92 I) p. 2 I $8-270$. [13, I 4 ].

(3) Über die Randwerte analytischer Funktionen. (Vorläufige Mitteilung.)

Sitzungsberichte Berl. Math. Ges., t. 20 (1921) p. 20-22. [22].

(4) Über die Randwerte einer analytischen Funktionen.

Math. Annalen, t. 84 (I92I) p. $232-244$. [22].

10-2822. Acta mathematica. 52. Imprimé le 25 janvier 1929. 
(5) Über die Mittelwerte analytischer Funktionen. Tôhuku Math. Journal, t. 22 (1922) p. 87-98. [22].

(6) Über Potenzreihen mit endlich vielen verschiedenen Koeffizienten. Berliner Sitzungsberichte, I 922, p. $88-91 .[23,24]$.

(7) Tschebyscheffsche Polynome und nichtfortsetzbare Potenzreihen. Math. Annalen, t. 87 (1922) p. 90-I I I. [23, 24$]$.

\section{S. Takenaka.}

(r) On the orthogonal functions and a new formula of interpolation. Japanese Journal of Math., t. 2 (I926) p. I29-- I45. [13, I4].

(2) On the representation of functions by the series of orthogonal functions. Japanese Journal of Math., t. 3 (1926) p. 27-36. [r3].

(3) On some properties of orthogonal functions associated to a closed curve on a complex plane.

Japanese Journal of Math., t. 3 (1926) p. 43-47. [I3].

\section{A. Tauber.}

(1) Über den Zusammenhang des reellen und imaginären Theiles einer Potenzreihe. Monatshefte f. Math. u. Physik, t. 2 (1891) p. 79-1 18. [22].

(2) Ein Satz aus der Theorie der unendlichen Reihen.

Monatshefte für Math. und Physik, t. 8 (1897) p. 273-277. [22, 33].

\section{W. Thomé.}

(I) Ueber Convergenz und Divergenz der Potenzreihe auf dem Convergenzkreise. Journal für Math., t. roo (1887) p. I67-178. [22].

(2) Über asymptotische Darstellungen von Functionen.

Journal für Math., t. 124 (1902) p. I $52-\mathrm{r} 56$. [34]

\section{Tillinger.}

(I) A transcendens egész függvények növekedésének vizsgálata, kapcsolatban az analytikai függvények szinguláritásainak vizsgálatával. [= Étude de la croissance des fonctions entières transcendentes en relation avec l'étude des singularités des fonctions analytiques.]

Math. és phys. lapok, t. 23 (I9I4) p. $72-8$ I, II $8-$ I $_{43}$ et I69-I 83 . [26,32].

\section{O. Toeplitz.}

(ז) Über allgemeine lineare Mittelbildungen.

Prace Mat.Fiz., t. 22 (1911) p. I I3-I I9. [30].

(2) Über das Wachstum der Potenzreihen im ihrem Konvergenzkreise. I.

Math. Zeitschrift, t. I 2 ( I 922 ) p. 189-200. [22]. 


\section{Tonelli.}

( I) I polinomi d'approssimazione di Tchebychev. Annali di Matematica, série 3, t. I5 (1908) p. 47-1 I9. [r5].

(2) Sulle serie di funzioni analitiche della forma $\Sigma a_{n}(x) x^{n}$. Annali di Mat., 3:e série, t. r8 (I9 I I) p. 99-I33. [1 3, I 4, 25 ].

\section{Tsuji.}

(1) On a power series which has only algebraic singularities on its convergence circle.

Japanese Journal of Math., t. 3 (1926) p. 69-85. [22, 24$]$.

\section{P. Urysohn.}

(I) Sur une fonction analytique partout continue. Fundamenta Math., t. 4 (I923) p. I 44-I 50. [13].

(2) Exemple d'une série entière prenant, sur son cercle de convergence, un ensemble de valeurs non mesurable $B$.

Comptes Rendus, t. 183 (4 Oct. I926) p. 548-550. [22].

\section{G. Valiron.}

(I) Sur le développement de Taylor d'une fonction méromorphe.

Nouvelles Annales de Math., 4:e série, t. Ir (r9ir) p. r8--20. [24].

(2) Remarques sur la sommation des séries divergentes par les méthodes de M. Borel. Rendiconti Circ. Mat. di Palermo, t. 42 (I9I7) p. 267-284. [31, 32, 33].

(3) Fonctions entières et fonctions méromorphes d'une variable. Mémorial Sci. Math., fasc. II, I 925,58 p., $8^{\circ}$. [I3].

\section{Ch. de la Vallée-Poussin.}

(r) Sur l'approximation des fonctions d'une variable réelle et de leurs dérivées par des polynomes et de suites limitées de Fourier.

Acad. R. de Belgique. Bull. classe sci. r9o8, p. I93-254. [3 I].

(2) Sur les polynomes d'approximation à une variable complexe. Ac. R. de Belgique. Bulletin sci. I9 I I, p. r99-2 I I. [ I5].

(3) Leçons sur l'approximation des fonctions d'une variable réelle. Paris, Gauthier-Villars, I 9 I 9, VI + I 5 I p., $8^{\circ}$. [ [ I 4, I 5$]$.

\section{E. B. Van Vleck.}

(1) On linear criteria for the determination of the radius of convergence of a power series.

Transactions Amer. Math. Soc., t. I (1 900) p. 293-309. [2 I, 24].

(2) Selected topics in the theory of divergent series and of continued fractions.

The Boston Colloquium I903. New York, Mac Millan, 1905, p. 75-187. [31, 34]. 
Einar Hille.

\section{T. Vijayaraghavan.}

(I) A Tauberian theorem.

Journal London Math. Soc., t. I (1926) p. II3-I20, 192. [33].

\section{A. Viterbi.}

(I) Sulla continuazione analitica delle funzioni monogene uniformi rappresentate col metodo del Mittag-Leffler.

Rendiconti Circ. Mat. di Palermo, t. I 2 (1898) p. 95-I Io. [I3, 25 ].

\section{G. Vivanti.}

(r) Sulle serie di potenze $\mathrm{i}$ cui coefficienti dipendono da una variabile. Annali di Mat., 3:e série, t. 2 I (I893) p. 25-32. [24].

(2) Sulle serie di potenze. (Estratto d'una lettera al prof. S. Pincherle.) Annali di Mat., 3:e série, t. 2 I (1893) p. 193-194. [24].

(3) Sulle serie di potenze.

Rivista di Mat., t. 3 (1893) p. I I I-II $4 .[22,23]$.

(4) Teoria delle funzioni analitiche.

Milano, U. Hoepli (Manuali Hoepli, 3 г $2-3$ I 3 ) (190I) VIII $+43^{2}$ p., I $2^{\circ}$. [ro, 20$]$.

(5) Theorie der eindeutigen analytischen Funktionen. Umarbeitung von A. Gutzmer. Leipzig, B. G. Teubner, I 906, VI +512 p., $8^{\circ}$. [1०, 20].

\section{Volterra.}

(I) Sopra alcune applicazioni della rappresentazione analitica delle funzioni del Prof. Mittag-Leffler.

Atti R. Ac. di Torino, t. 34 (1899) p. 492-494. [27].

\section{J. L. Walsh.}

(I) On the expansion of analytic functions in series of polynomials. Transactions Amer. Math. Soc., t. $26(1924)$ I 55-i 70 . [ [ 13. I 4 ].

(2) Sur la détermination d'une fonction analytique par ses valeurs sur un contour. Comptes Rendus, t. 178 (2 Janvier 1924) p. 58-59. [ $\mathrm{r}_{3}$ ].

(3) Über die Entwicklung einer analytischen Funktion nach Polynomen. Math. Annalen, t. 96 ( 1926$)$ p. $430--436$. [13, 14$]$.

(4) Über die Entwicklung einer Funktion einer komplexen Veränderlichell nach Polynomen.

Math. Annalen, t. 96 (1926) p. $437-450$. [1 4].

\section{A. Walther.}

(I) Über den Cauchy-Hadamardschen Satz vom Convergenzradius; nebst einer Darstellung der Dedekindschen Irrationalzahlentheorie.

Monatshefte f. Math. u. Phys., t. I2 (I90I) p. 49-8I. [2 I]. 
Essai d'une bibliographie de la représentation analytique d'une fonction monogène.

\section{Watanabe.}

(r) On the identity of Cesàro's and Hölder's limits. Tôhuku Math. Journal, t. 5 (I 9 I 4 ) p. 2 I -28 . [3I].

\section{G. N. Watson.}

(I) The singularities of fonctions defined by Taylor's series. Quarterly Journal of Math., t. 42 (I9I0) p. 4I-53. [23,3 I].

(2) A theory of asymptotic series.

Phil. Transactions R. Soc. London, série A, t. 2 I I (I9II) p. 279-3 I3. [34].

(3) The characteristics of asymptotic series.

Quarterly Journal, t. 43 ( I91 r) p. 63-77. $\left[3^{2}, 34\right]$.

\section{R. Wavre.}

(r) Sur les développements de Mittag-Leffler. Comptes Rendus des séances de la Soc. Math. de France, 1920, p. 53. [25].

(2) Sur les développements d'une fonction analytique en série de polynomes. L'enseignement math., t. 2 I (1920) p. $227-228$. [25].

\section{K. Weierstrass.}

(1) Zur Theorie der Potenzreihen.

Math. Werke, t. I, p. $67-74$. [2 I].

(2) Zur Theorie der eindeutigen analytischen Functionen.

Berliner Abhandlungen 1876 , p. I $\mathbf{I}-60$. Abhandlungen aus der Functionenlehre, p. I-52. Math. Werke, t. 2, p. $77-124$. [I3].

(3) Mémoire sur les fonctions analytiques uniformes. Traduit par É. Picard. Annales de l'École Normale, 2 :e série, t. 8 (1879) p. I I I-I 50. [13].

(4) Ueber einen functionentheoretischen Satz des Herrn G. Mittag-Leffler. Berliner Monatsberichte 1880 p. 707-717. Abhandlungen aus der Functionenlehre, p. 53-66. Math. Werke, t. 2, p. 189-199. [13].

(5) Zur Functionenlehre. Berliner Monatsberichte, 1880 p. 7 19-743. Abhandlungen aus der Functionenlehre, p. 67-IоI. Math. Werke, t. 2, p. 20I-223, 224-230. [13].

(6) Mitteilung zur Functionenlehre.

Berliner Monatsberichte, I88 I p. 228-230. Abhandlungen aus der Functionenlehre, p. I02-I04. Math. Werke, t. 2, p. 23r-233. [13].

(7) Sur un théorème de M. Mittag-Leffler et sur la théorie des fonctions uniformes. Bulletin d. Sci. Math., série 2, t. 5 (I88I) p. I I 3-1 24 . [I3].

(8) Remarques sur quelques points de la théorie des fonctions analytiques. Bulletin d. Șci. Math., série 2, t. 5 (I881) p. I57-I83. [I3].

(9) Abhandlungen aus der Functionenlehre.

Berlin, J. Springer, $1886, \mathrm{IV}+262$ p. gr. $8^{\circ}$. [I 3 ]. 
( 10$)$ Zur Functionentheorie.

Acta Math., t. $45(1925)$ p. I- Io. [Io].

\section{Weniaminoff.}

(r) Sur la dérivée-limite d'une fonction analytique.

Comptes Rendus, t. I 80 (I 2 Janvier I925) p. I I4-i I6. [22].

\section{N. Wiener.}

(r) Note on a new type of summability.

American Journal of Math., t. 45 (I923) p. $83-86$. [3 r].

\section{S. Wigert.}

(1) Sur les fonctions entières.

Öfversigt K. Vetenskaps-Akad. Förhandl., t. 57 (r900) p. I00I--101 r. [24].

(2) Sur un théorème de la théorie des fonctions analytiques. Arkiv för Mat., Astr. o. Fys., t. 5, n:o 8 (r9o9) ro p. [24].

(3) Sur la convergence de deux séries associées. Arkiv för Mat., Astr. o. Fys., t. I I, n:o 5 (1916) I 2 p. [33].

(4) Sur une certaine classe de séries de puissances.

Arkiv för Mat., Astr. o. Fys., t. I 2, n:o 7 (I9I7) I 3 p. [24].

\section{E. J. Wilczynski.}

(1) On the form of the power series for an algebraic function.

American Math. Monthly, t. 26 (1919) p. 9-1 2. [24].

\section{A. Wiman.}

(I) Über den Fundamentalsatz in der Theorie der Funktionen $\boldsymbol{E}_{\boldsymbol{\alpha}}(x)$.

Acta Math., t. 29 (1905) p. 19I-201. [32].

(2) Über die Nullstellen der Funktionen $E_{c(}(x)$.

Acta Math., t. 29 (I905) p. 2 I $7-234$. [32].

\section{W. Wirtinger.}

(1) Einige Anwendungen der Euler-Maclaurin'schen Summenformel, insbesondere auf eine Aufgabe von Abel.

Acta Math., t. 26 (1902) p. 255-27 I. [22, 24$]$.

(1) Sur les séries $\sum \frac{A_{k}}{z-\alpha_{k}}$.

\section{J. Wolff.}

Comptes Rendus, t. I 73 (28 Novembre I92 I) p. I056- I057 et (I 7 Décembre I 92 I) p. I 327 - 328 . [I 3, I 4,24$]$. 
Essai d'une bibliographie de la représentation analytique d'une fonction monogène.

\section{W. H. Young.}

(1) Über eine Summationsmethode für die Fouriersche Reihe. Leipziger Berichte, t. 63 (r9r r) p. $369^{-3} 37$. [3r].

(2) On infinite integrals involving a generalisation of the sine and cosine functions. Quarterly Journal of Math., t. 43 (I9I2) p. I6I-I77. [3I].

(3) On restricted Fourier series and the convergence of power series. Proceedings London Math. Soc., série 2, t. I 7 (I9I8) p. 353-366. [22, 33].

\section{Zoretti.}

(I) Sur les ensembles parfaits et les fonctions uniformes. Comptes Rendus, t. $3_{38}$ (I 4 Mars I 904) p. 674-676. [I3].

(2) Sur les singularités des fonctions analytiques. Comptes Rendus, t. $3_{3}$ (25 Avril r 904) p. I026-1027. [I3].

(3) Sur les fonctions analytiques uniformes qui possèdent un ensemble parfait discontinu de points singuliers.

Journal de Math., série 6, t. I (1905). p. I-5 r. Thèse, Paris, Gauthier-Villars. [r3].

(4) Sur le développement d'une fonction analytique uniforme en produit infini. Comptes Rendus, t. I4I (I3 Novembre I905) p. 753-754. [ I3].

(5) Sur un ensemble de la théorie des fonctions analytiques.

Bull. Sci. Math., série 2, t. 29 (1905) p. 276-277. [I3].

(6) Leçons sur le prolongement analytique.

Paris, Gauthier-Villars, I 9 I I, VI +116 p. gr. $8^{\circ} .[10,12,13]$.

\section{A. Zygmund}

( 1$)$ Sur une généralisation de la méthode de Cesàro. Comptes Rendus, t. I 79 (3 Novembre I 924 ) p. 870-872. $[22,3 \mathrm{I}]$.

(2) Sur la sommation des séries par le procédé des moyennes typiques. Bulletin int. Acad. Sci. de Cracovie, A. I $9^{2} 5$, p. $26_{5}-287$. $[3$ I, 33].

(3) Sur la sommation des séries trigonométriques et celles de puissances par les moyennes typiques.

Comptes Rendus, t. I 8 I (28 Décembre I 925 ) p. I I 22- I I $23 .[22,3$ I].

(4) Sur la théorie riemannienne des séries trigonométriques.

Math. Zeitschrift, t. 24 (1926) p. 47-104. [22].

(5) Sur un théorème de la théorie de la sommabilité.

Math. Zeitschrift, t. 25 (1926) p. 291-296. [22, 31]. 


\section{A D D E N D A. ${ }^{1}$}

\section{S. Dobrowolski.}

(I) Sur les valeurs limites de moyennes de Cesàro et de Hölder.

Bulletin int. Acad. Sei. de Cracovie, A. I 925 , p. $259^{-264}$ [3I].

W. Fédoroff.

(I) Sur la continuité des fonctions analytiques.

Mat. Sbornik, t. 32 (I924) p. I I 5-I 2 I. [I 3 ].

(2) O proizvodnoj analitičeskoj funkcii vblizi množestva osobich toček. [= Sur la dérivée d'une fonction analytique dans le voisinage d'un ensemble de points singuliers.]

Mat. Sbornik, t. 32 (1924) p. I22-- 34 . [1 3$]$.

\section{A. Haar.}

(2) Über asymptotische Entwicklungen von Funktionen.

Math. Annalen, t. $96(1926)$ p. $69-107 . \quad[34,35]$.

\section{A. Maksymowicz.}

(r) Contribution à la théorie des séries sommables par la méthode de Cesaro-Hölder. [Résumé?]

Bulletin int. Acad. Sci. de Cracovie, A. I 920 , p. 65-69. $[24,33]$.

\section{G. Mignosi.}

(3) Estensione dei teoremi di Abel, Cesaro e Frobenius sulle serie di potenze, I e II. Rendiconto Accad. Sci. di Napoli, 3 :e série, t. 26 (1920) p. $225^{-235}, 237-246[24]$.

\section{A. Rajchman et A. Zygmund}

(I) Sur la relation du procédé de sommation de Cesàro et celui de Riemann. Bulletin int. Acad. Sci. de Cracovie, A. I925, p. 69-80. [3 I, 33].

${ }^{1}$ Pendant la correction des épreuves, j'ai ajouté plusieurs ouvrages qui m'avaient echappé auparavant pour une raison ou pour une autre. Les ouvrages que je n'ai pas pu faire figurer à leur place normale ont été placés dans cet addenda. Je n'ai fait aucun effort pour introdnire dans cette bibliographie les ouvrages publiés pendant les deux années qui ont suivi sa rédaction. 